\title{
A new species concept for the clinically relevant Mucor circinelloides complex
}

\author{
L. Wagner ${ }^{1}$, J.B. Stielow ${ }^{2,3}$, G.S. de Hoog ${ }^{2,4}$, K. Bensch ${ }^{2,5}$, V.U. Schwartze ${ }^{6,7}$, \\ K. Voigt ${ }^{6,7}$, A. Alastruey-Izquierdo ${ }^{8}$, O. Kurzai ${ }^{1,9}$, G. Walther ${ }^{1}$
}

Key words

mating tests

maximum growth temperature

Mucor

mucormycosis

new taxa

phylogeny

taxonomy

zygospore formation

\begin{abstract}
Mucor species are common soil fungi but also known as agents of human infections (mucormycosis) and used in food production and biotechnology. Mucor circinelloides is the Mucor species that is most frequently isolated from clinical sources. The taxonomy of Mucor circinelloides and its close relatives (Mucor circinelloides complex - MCC) is still based on morphology and mating behaviour. The aim of the present study was a revised taxonomy of the MCC using a polyphasic approach. Using a set of 100 strains molecular phylogenetic analysis of five markers (ITS, rpb1, ts $1, \mathrm{mcm} 7$, and $c f s$, introduced here) were performed, combined with phenotypic studies, mating tests and the determination of the maximum growth temperatures. The multi-locus analyses revealed 16 phylogenetic species of which 14 showed distinct phenotypical traits and were recognised as discrete species. Five of these species are introduced as novel taxa: $M$. amethystinus sp. nov., $M$. atramentarius sp. nov., $M$. variicolumellatus sp. nov., M. pseudocircinelloides sp. nov., and $M$. pseudolusitanicus sp. nov. The former formae of $M$. circinelloides represent one or two separate species. In the MCC, the simple presence of well-shaped zygospores only indicates a close relation of both strains, but not necessarily conspecificity. Seven species of the MCC have been implemented in human infection: $M$. circinelloides, $M$. griseocyanus, $M$. janssenii, $M$. lusitanicus, $M$. ramosissimus, $M$. variicolumellatus, and $M$. velutinosus.
\end{abstract}

Article info Received: 13 November 2018; Accepted: 10 April 2019; Published: 29 August 2019.

\section{INTRODUCTION}

Mucor is a large genus within the Mucorales, comprising mainly saprotrophs occurring in soil and dung, but also endophytes and parasites of plants and other fungi (Domsch et al. 1980, Walther et al. 2013). Mucor species are used in biotechnology as biotransformants or as producers of enzymes and diverse metabolites. They also play a role in the production of food, e.g., in the fermentation of traditional Asian soybean-based food (Han et al. 2004, Hong et al. 2012) and European cheese (Hermet et al. 2012) and they are important spoilage organisms of a large variety of raw materials and processed food (MorinSardin et al. 2017).

Mucor, Rhizopus, Lichtheimia, and Apophysomyces are the main genera of Mucorales potentially causing mucormycosis (Petrikkos et al. 2012), a gamut of opportunistic human infections ranging from chronic cutaneous to rhinocerebral. When

\footnotetext{
1 German National Reference Center for Invasive Fungal Infections, Leibniz Institute for Natural Product Research and Infection Biology - Hans Knöll Institute, Jena, Germany;

corresponding author e-mail: Grit.Walther@leibniz-hki.de.

2 Westerdijk Fungal Biodiversity Institute, Utrecht, The Netherlands

3 Thermo Fisher Diagnostics, Speciality Diagnostics Group, Landsmeer, The Netherlands.

${ }^{4}$ Center of Expertise in Mycology of Radboud University Medical Center Canisius Wilhelmina Hospital, Nijmegen, The Netherlands.

${ }^{5}$ Botanische Staatssammlung München, München, Germany.

6 Jena Microbial Resource Collection, Leibniz Institute for Natural Product Research and Infection Biology - Hans Knöll Institute, Jena, Germany.

7 Faculty of Biology and Pharmacy, Institute of Microbiology, Department Microbiology and Molecular Biology, Friedrich Schiller University Jena, Jena, Germany.

${ }^{8}$ Mycology Reference Laboratory, National Centre for Microbiology, Instituto de Salud Carlos III, Majadahonda, Madrid, Spain.

${ }^{9}$ Institute for Hygiene and Microbiology, University of Würzburg, Würzburg, Germany.
}

patients have a severe underlying disease, the infection may take a dramatic course with high mortality rates. Risk factors for systemic infection are uncontrolled diabetes, deep neutropenia, and long-term use of steroids as therapy for cancer or organ transplantation (Greenberg et al. 2004, Chayakulkeeree et al. 2006).

Members of the Mucor circinelloides complex (MCC) as defined by Walther et al. (2013) are among the Mucor species that are most frequently isolated from clinical sources (Walther et al. 2013) and that have been proven to be involved in infections (Álvarez et al. 2009, 2011, De Hoog et al. 2016). In patients without underlying diseases, the MCC usually causes cutaneous or subcutaneous infections (Morin-Sardin et al. 2016) or infections related to extensive burns (Garcia-Hermoso et al. 2018), or deep trauma (Vitrat-Hincky et al. 2009). Systemic infections caused by the MCC have been reported in patients with impaired immunity due to hematologic malignancies (Fingeroth et al. 1994, Chandra \& Woodgyer 2002, Iwen et al. 2007, Sugui et al. 2011, Racsa et al. 2016), uncontrolled diabetes (Dizbay et al. 2009) or severe intestinal dysfunctions (Chan-Tack et al. 2005, Kumar et al. 2018). Contamination of food by $M$. circinelloides can cause gastrointestinal disorders (Lee et al. 2014). Pulmonary infection are mostly caused by Rhizopus and Lichtheimia (Tedder et al. 1994) rather than by Mucor species. In the period January 2014 to August 2018 the German National Reference Center for Invasive Fungal Infections registered 10 cases of Mucor infection that were all caused by Mucor circinelloides f. circinelloides: seven cases were related to deep trauma and two to burns.

In contrast to Rhizopus (Liu et al. 2008, Abe et al. 2010, Dolatabadi et al. 2014a, b), Lichtheimia (Alastruey-Izquierdo et al. 2010), and Apophysomyces (Álvarez et al. 2010) where modern species concepts were applied based on molecular phylo- 
genies, the species-rich, polyphyletic genus Mucor lacks a state-of-the-art taxonomy. Recent taxonomic studies are limited to species occurring in particular habitats, such as clinical samples (Álvarez et al. 2011) or cheese (Hermet et al. 2012), and consequently do not cover the complete diversity of the fungi concerned.

The last taxonomic revision of the MCC dates back to 1976 (Schipper 1976b). Based on morphology and mating abilities, Schipper (1976b) recognised four formae within Mucor circine/loides, i.e., forma circinelloides, f. griseocyanus, f. janssenii, and f. lusitanicus. In DNA barcoding studies using the nuclear ITS region (Walther et al. 2013), these taxa formed well-supported clades, but were found to be intermingled with supposedly unrelated species, such as $M$. bainieri, $M$. ctenidius, and $M$. ramosissimus, as well as Ellisomyces anomalus. In addition, several strains with $M$. circinelloides morphology were not part of one of the four clades matching with Schipper's formae.

Since then, the taxonomic status of the formae has remained controversial. Analyses of low numbers of strains resulted in discrete topologies, which the authors considered to represent the formae and which were sufficiently remote from each other to be regarded as discrete species (Álvarez et al. 2011, Li et al. 2011). However, phylogenetic analysis of larger numbers of strains resulted in a more complex topology, with numerous strains that could not clearly be assigned to any of the formae (Walther et al. 2013). Because of these intermediary strains, the occurrence of successful mating between the formae, and the absence of compensatory base changes, Walther et al. (2013) maintained the status of formae for the clades. Mucor velutinosus was newly described from clinical specimens (Álvarez et al. 2011), but the high ITS sequence similarity with M. circinelloides f. janssenii led Walther et al. (2013) to treat this species as a synonym of the latter.

Genealogical concordance phylogenetic species recognition (GCPSP) (Taylor et al. 2000) is the current standard method for defining species boundaries in fungi (Hibbett \& Taylor 2013). However, the Mucorales are a phylogenetically ancient group, which is reflected in high sequence variability even within species (Walther et al. 2013). Therefore, multi-locus analyses bear the risk to overestimate the value of markers and the number of extant species. In order to increase the reliability of species boundaries we therefore combined multi-locus studies with mating tests and morphological studies.

Mating tests have traditionally been used in the Mucorales to define biological species boundaries (e.g., Schipper 1976b). Crosses between different, but closely related species may result in the induction of azygospore formation (O'Donnell et al. 1977a), or lead to production of zygospores that differ in size, colour, ornamentation or number from intraspecific zygospores (Alastruey-Izquierdo et al. 2010, Schell et al. 2011). Mature azygospores may be similar to true zygospores by wall ornamentation and may have two suspensors, but lysis of the fusion septum between the gametangia remains absent and no plasmogamy takes place (O'Donnell et al. 1977a). The use of zygospore formation to define species borderlines is controversial because interspecific zygospores are known and only the formation of the zygospores but not their germination is observed in most cases. Only few authors observed germination of zygospores in Mucor (Gauger 1961, 1965, James \& Gauger 1982, Schell et al. 2011) and to our knowledge, no such reports exist for the MCC. However, vitality of zygospores with production of a fertile sporangium provides crucial evidence for biological species recognition. In the present study we therefore not only studied the formation but also the germination of zygospores.

Members of the MCC are closely related to $M$. racemosus and its allies $M$. plumbeus and $M$. brunneogriseus, in the following referred to as the $M$. racemosus complex (MRC). MCC and $\mathrm{MRC}$ form a monophyletic group and share a similar morphology, which is why they were combined into the Mucor racemosus group (Walther et al. 2013). In order to allow a reliable phenotypic identification of these complexes our morphological study also included members of the MRC.

Due to the frequent occurrence of paralogs in the genome, the use of established phylogenetic markers is limited in Mucorales (Alastruey-Izquierdo et al. 2010). In addition to the nuclear ribosomal internal transcribed spacer (ITS), the gene of the mini-chromosome maintenance complex component 7 ( $\mathrm{mcm} 7)$, the largest subunit of RNA polymerase II (rpb1), and the 20S rRNA accumulation protein (tsr1), we therefore introduced a new marker predicted to encode for a cyclopropane-fattyacylphospholipid-synthase (cfs) that was found by mining four mucoralean genomes.

The aim of this study was to establish a modern polyphasic taxonomy of the MCC by applying a five-locus phylogeny, mating tests, in addition to morphological and physiological characters. Fourteen out of 16 recognised phylogenetic species showed distinct phenotypic characters and were accepted as discrete species. Five species in the MCC were newly described. Importantly, the number and appearance of zygospores did not allow unambiguous decisions on conspecificity. A key to all accepted species of the MCC is provided, allowing species identification without the use of molecular tools. Clinically relevant species of the MCC are $M$. circinelloides, $M$. velutinosus, $M$. griseocyanus, $M$. janssenii, $M$. lusitanicus, $M$. ramosissimus, and $M$. variicolumellatus, with most cases caused by the first two species.

\section{MATERIALS AND METHODS}

\section{Strains}

A total of 100 strains originating from the Westerdijk Fungal Biodiversity Institute (CBS, The Netherlands), the Jena Microbial Resource Collection (JMRC:FSU, Germany), the Instituto de Salud Carlos III National Centre for Microbiology (CNM-CM, Spain) and the Belgian Co-ordinated collection of Microorganism (IHEM, Belgium) were included in the study (Table 1). Ninety five strains belong to the MCC as defined by Walther et al. (2013): Mucor circinelloides f. circinelloides (46 strains), M. circinelloides f. griseocyanus (8), M. circinelloides f. janssenii (11), $M$. circinelloides f. lusitanicus (15), $M$. ctenidius (3), M. ramosissimus (1), M. bainieri (1), Mucor sp. (8) and Ellisomyces anomalus (2) (Table 1). Five strains represent the MRC: $M$. brunneogriseus CBS 213.75, M. plumbeus CBS 226.32, M. racemosus f. racemosus CBS 260.68, CBS 774.86, M. racemosus $\mathrm{f}$. sphaerosporus CBS 115.08 and were included for comparative morphological analyses.

\section{Morphology}

Strains were grown at room temperature on two types of malt extract agar: 1) MEA: $40 \mathrm{~g} / \mathrm{L}$ malt extract (Becton Dickinson, Heidelberg, Germany), yeast extract $4 \mathrm{~g} / \mathrm{L}$ (Ohly, Hamburg, Germany), $1.5 \%$ agar (Europäischer Agar, Otto Nordwald, Hamburg, Germany) and 2) MEX: $15 \mathrm{~g} / \mathrm{L}$ malt extract (Becton Dickinson, containing $6 \mathrm{~g}$ malt extract base, $1.8 \mathrm{~g}$ maltose, $6 \mathrm{~g}$ Bacto glucose, $1.2 \mathrm{~g}$ Bacto yeast extract) and $1.5 \%$ agar (Europäischer Agar, Otto Nordwald), as well as on potato dextrose agar (PDA: $39 \mathrm{~g} / \mathrm{L}$, Roth, Karlsruhe, Germany) and synthetic Mucor agar (Benny 2008) (SMA: $40 \mathrm{~g} / \mathrm{L}$ dextrose (Roth), $2 \mathrm{~g} / \mathrm{L}$ asparagine (Reanal Laborvegyszer Kereskedelmi Kft., Budapest, Hungary), $0.5 \mathrm{~g} / \mathrm{L} \mathrm{KH}_{2} \mathrm{PO}_{4}$ (Merck, Darmstadt, Germany), $0.25 \mathrm{~g} / \mathrm{L} \mathrm{MgSO}_{4} \times 7 \mathrm{H}_{2} \mathrm{O}$ (Roth), $0.5 \mathrm{mg} / \mathrm{L}$ thiamine hydrochloride (Roth), $1.5 \%$ agar (Europäischer Agar, Otto Nordwald). Macroscopic and microscopic features were studied after 3 and $7 \mathrm{~d}$ 


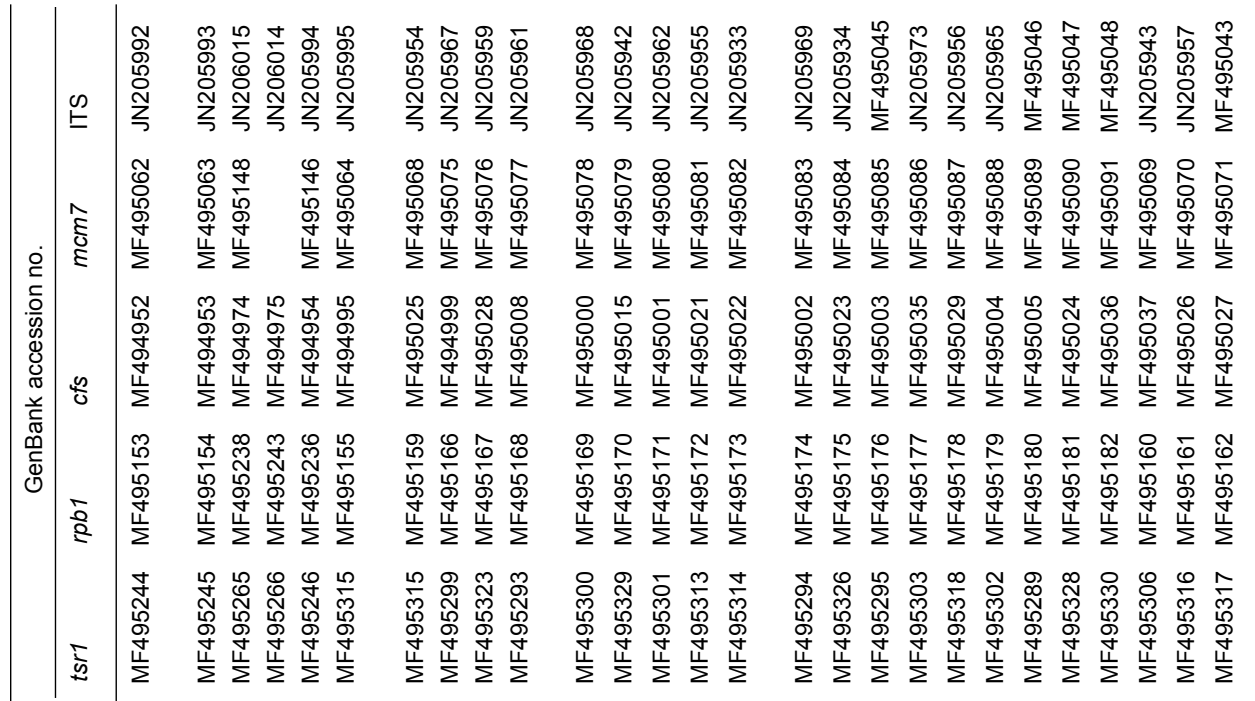

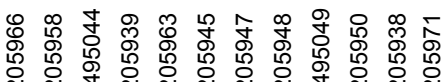

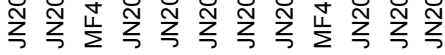

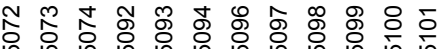

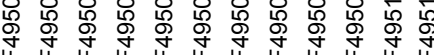

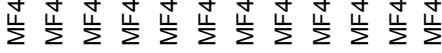

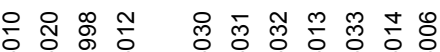

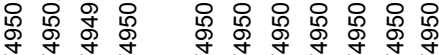

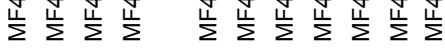

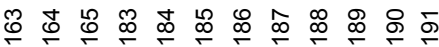

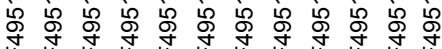

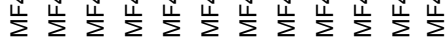

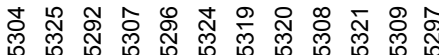

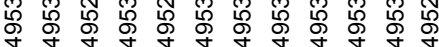

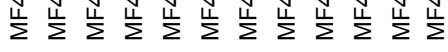

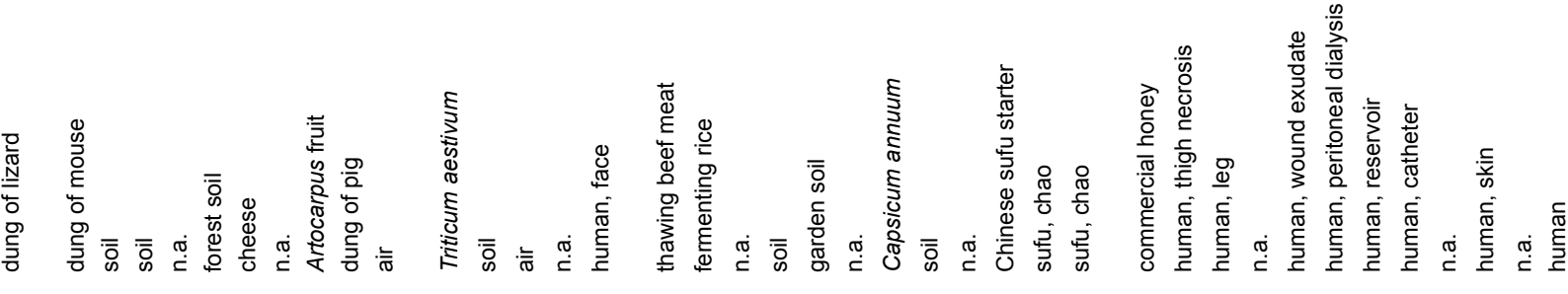

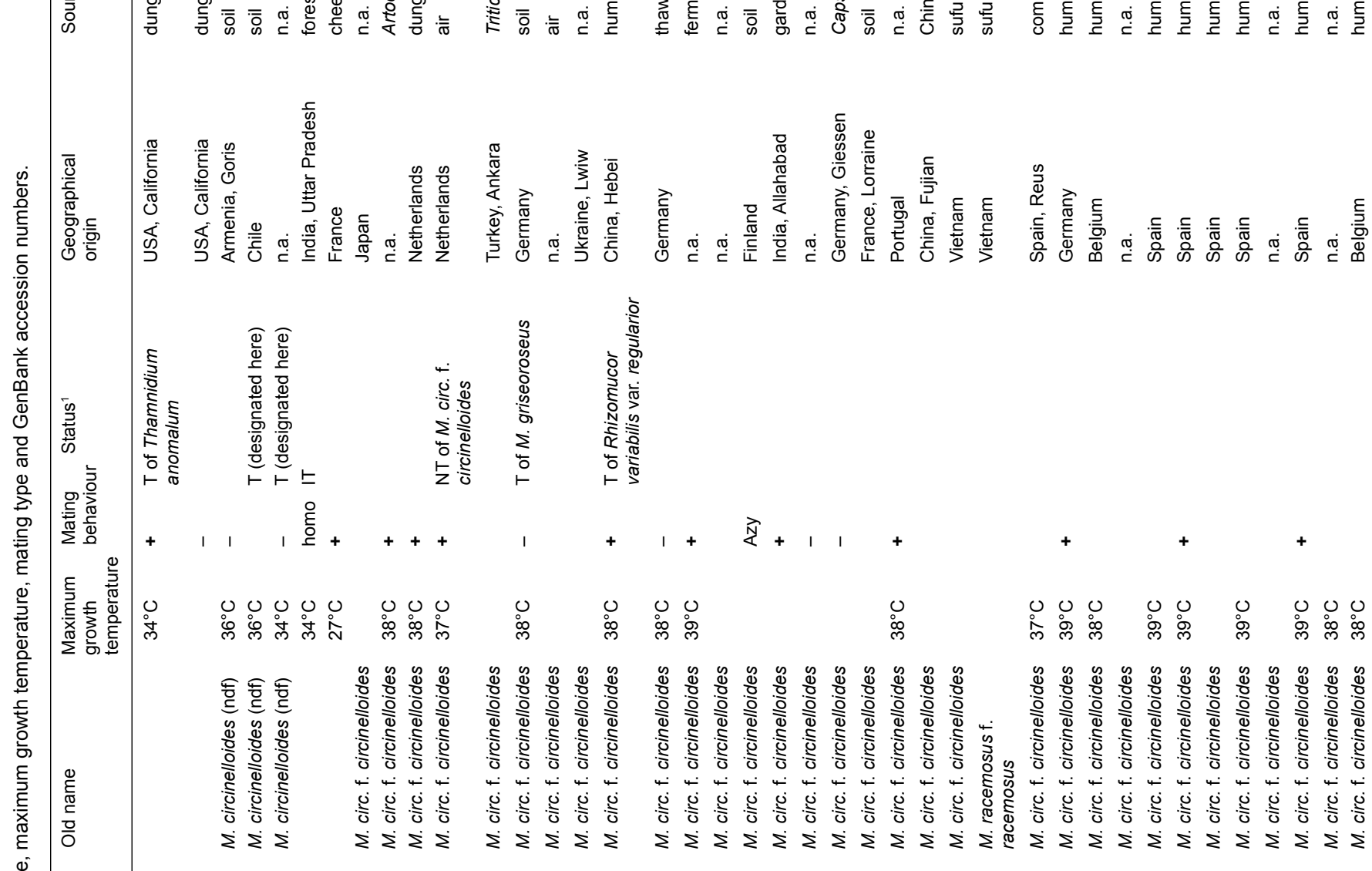




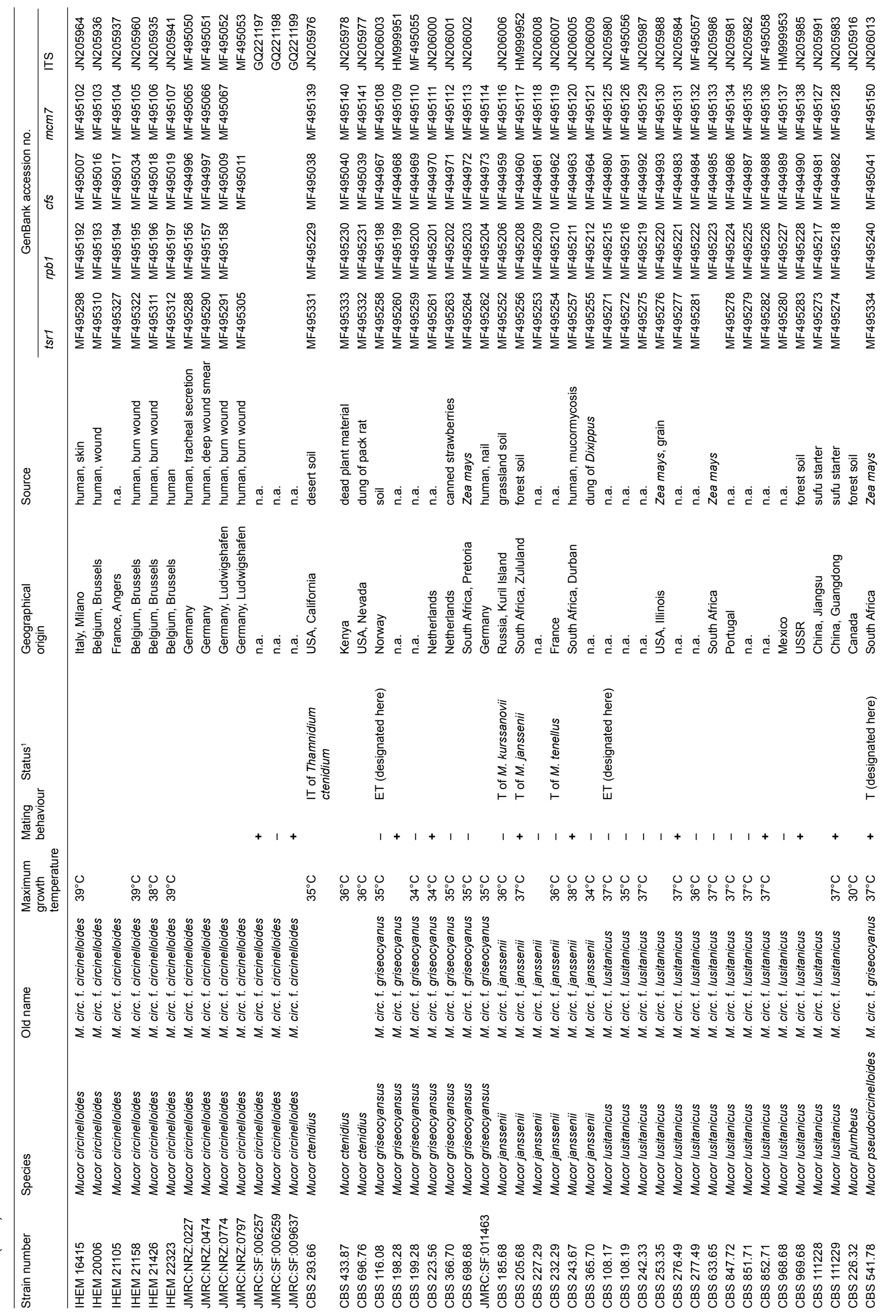


using a Zeiss Stemi 1000 (Carl Zeiss, Jena, Germany) and a Nikon Eclipse Ni Microscope (Nikon, Düsseldorf, Germany) with differential interference contrast and the NIS Elements software v. 4.30 (Nikon). Colours of the mycelia were described using the colour charts of Munsell (Anonymous 1990).

\section{Yeast growth}

The ability to grow as yeast was tested for all species. To induce yeast growth, spores from 3-7-d-old colonies on MEA were harvested. $100 \mathrm{~mL}$ Erlenmeyer flasks containing $50 \mathrm{~mL}$ yeast-peptone-glucose broth (YPG: $0.3 \%$ yeast extract (Ohly), $1 \%$ peptone (Roth), $3 \%$ glucose (Roth)) were inoculated with a final inoculum of $10^{5} \mathrm{spores} / \mathrm{mL}$ and incubated under nonshacking condition at room temperature. After 24 to $48 \mathrm{~h}$ the morphology was studied microscopically.

\section{Maximum growth temperature}

To determine the maximum temperature of growth, 3-d-old MEA cultures were used to inoculate MEA culture plates $(90 \mathrm{~mm}$ diam) at the centre. The plates were incubated at the respective temperature $\left(33-40^{\circ} \mathrm{C}\right)$ overnight. Colony margins were marked daily for $3 \mathrm{~d}$ and the rate of growth was determined based on the growth from day 1 to day 3 . Mycelia that grew only after inoculation but then cease to grow were considered as not growing.

\section{Mating}

Preliminary mating experiments were performed on MEA and on whey agar (WA, $20 \mathrm{~g} / \mathrm{L}$ powdered whey (Aurelia Allgäuer Naturprodukte, Weiler-Simmerberg, Germany), $10 \mathrm{~g} / \mathrm{L}$ glucose (Carl Roth, Karlsruhe, Germany)) in darkness for 4 wk. In subsequent experiments only WA was used because of higher rates of zygospore formation on this medium. Strains were pre-grown for $3 \mathrm{~d}$ on MEA at room temperature. Mycelial sections of about $0.5 \times 0.5 \mathrm{~cm}$ were placed on WA at a distance of $20 \mathrm{~mm}$ from the respective mating partner, close to the margin of the Petri dish. After 4 wk, plates were screened for zygospores using a Zeiss Stemi 1000 stereo microscope (Carl Zeiss, Jena, Germany). To enhance detection of zygospores, $95 \%$ ethanol was applied to remove sporangia. Numbers of zygospores per plate were counted or estimated (in case of high numbers) for each successful cross. Several zygospores per plate were moved to a slide using a sterile needle, and mounted in lactic acid (80\%). Diameters (without ornamentation because it was often flexed) and maximum height of ornamentation of at least 20 zygospores were measured using a Nikon Eclipse Ni Microscope (Nikon) with NIS Elements software v. 4.30 (Nikon).

Seven pairs of strains known to produce zygospores (Schipper 1976b) (CBS $172.27 \times$ CBS 116.08, CBS 192.68 × CBS 116.08, CBS $205.68 \times$ CBS 116.08, CBS $192.68 \times$ CBS 394.68, CBS $172.27 \times$ CBS 394.68, CBS $205.68 \times$ CBS 232.29, and CBS $243.67 \times$ CBS 232.29 , were included in preliminary mating tests in order to show that the applied conditions are appropriate for zygospore formation. Crosses of the reference strains were repeated in every mating experiment in view of reproducibility.

\section{Zygospore germination}

Two different approaches were used to observe zygospore germination. In the first approach zygospores with adherent hyphae and sporangiophores were harvested 4 wk after the start of the mating experiments in $1.5 \mathrm{~mL}$ tubes, dried for at least $1 \mathrm{wk}$ at room temperature, and washed twice in sterile water with $0.1 \%$ Tween 20 . The supernatant was removed after each washing step in order to reduce the amount of sporangiospores. Wet mycelia were spread repeatedly over cell strainers (pore size $70 \mu \mathrm{m}$; EASYstrainer GBO, Kremsmünster, Austria) using a pipette tip and rinsed with sterile water until 
zygospores were mechanically separated from mycelia. Subsequently, zygospore-containing filtrates were filtered again using cell strainers with a pore size of $40 \mu \mathrm{m}$ (EASYstrainer). Zygospores were subsequently rinsed repeatedly with sterile water with $0.1 \%$ Tween 20 and plated on MEA plates using a cell spreader. Cleaned zygospores were almost free of vegetative cell material. MEA plates containing the zygospores were examined after $12 \mathrm{~h}$. Fast-sprouting colonies originating from sporangiospores or hyphae were cut out from the plate or clean zygospores were transferred to a new culture plate using a dissecting needle and checked daily for sprouting hyphae or sporangiospores for $1 \mathrm{wk}$. If no sprouting was observed, zygospores were transferred to water agar $(15 \mathrm{~g} / \mathrm{L}$ agarose; Otto Nordwald), maintained at room temperature, and observed daily during the first week and then twice a week. In the second approach, the zygospores were already harvested after $10 \mathrm{~d}$ and directly separated from the mycelia without drying by applying the same washing steps as described for the first approach. The cleaned zygospores were soaked in sterile water at room temperature for $2 \mathrm{~d}$ and subsequently plated on agarose plates (1\%, Serva Electrophoresis $\mathrm{GmbH}$, Heidelberg, Germany) adjusted to $\mathrm{pH} 4$ (after sterilisation, with $\mathrm{HCl}$ ) (Guo \& Michailides 1998).

\section{DNA-extraction and sequencing}

Genomic DNA was extracted according to Möller et al. (1992) with modifications of Alastruey-Izquierdo et al. (2010). Marker genes were selected based on 1) amplification rates in Mucor; 2) absence of paralogs; and 3) sequence diversity (Tanabe et al. 2004, Crespo et al. 2007, Aguileta et al. 2008, Tretter et al. 2013), as follows: rDNA internal transcribed spacer (ITS) region, mini-chromosome maintenance complex component 7 ( $\mathrm{mcm} 7$, partial), the gene encoding the largest subunit of RNA polymerase II (rpb1, partial), the gene of 20S rRNA accumulation protein (tsr1, partial), and cyclopropane-fatty-acylphospholipidsynthase (cfs, partial), the latter found here by comparison of four mucoralean genomes (see below).

PCR was done in $25 \mu \mathrm{L}$ volumes with MyTaq ${ }^{\mathrm{TM}}$ DNA Polymerase (Bioline, Luckenwalde, Germany) ( $5 \mu \mathrm{L} 5 \times$ My Taq Reaction Buffer including $5 \mathrm{mM}$ deoxynucleoside triphosphates and $15 \mathrm{mM} \mathrm{MgCl}, 0.4 \mu \mathrm{M}$ for each primer (Table 2), 100-200 ng DNA, 0.625 U My Taq) using a Biometra T300 thermocycler (Analytic Jena, Jena, Germany): initial denaturation $95^{\circ} \mathrm{C}$ for $5 \mathrm{~min}, 30$ cycles of denaturation $\left(95^{\circ} \mathrm{C}\right.$ for $30 \mathrm{~s}$ ), annealing (temperature according to the primer for $30 \mathrm{~s}$, Table 2), and extension $\left(72^{\circ} \mathrm{C}\right.$ for $45 \mathrm{~s}$ ), final extension $72{ }^{\circ} \mathrm{C}$ for $7 \mathrm{~min}$. All primers were synthesized by Biomers.net (UIm, Germany).

If direct sequencing of amplification products was unsuccessful, PCR products were cloned using the pGEM-T Easy Vector
System (Promega GmbH, Mannheim, Germany). Cloned sequences were amplified by colony $\mathrm{PCR}$ using M13 primers (M13f: 5'-GTAAAACGACGGCCAGT-3', M13r: 5'-GGAAACAGCTATGACCATG-3'). PCR products were purified by means of the Pure Link ${ }^{\mathrm{TM}}$ Quick PCR Purification Kit (Thermo Fisher Scientific, Schwerte, Germany) and DNA purification columns (Genaxxon Bioscience, Ulm, Germany). A part of the ITS and LSU sequences were generated at Westerdijk Institute (CBSKNAW). Molecular work and methods performed for this part, i.e., DNA purification, PCR setup, Sanger sequencing data production and its curation is fully referenced in Stielow et al. (2015).

\section{Genome mining for phylogenetic markers}

Four annotated genomes of mucoralean species, i.e., Mucor circinelloides CBS 277.49 v. 2.0 (Lee et al. 2014, Corrochano et al. 2016), Rhizopus arrhizus var. delemar strain 99-880 (Ma et al. 2009), Lichtheimia corymbifera JMRC:FSU:9682 (CBS 429.75, ATCC 46771) (Schwartze et al. 2014), Phycomyces blakesleansus NRRL 1555 v. 2.0 (Corrochano et al. 2016), were compared for homologous genes based on GhostFam homology families (Schwartze et al. 2014, GhostFam default parameter settings: $50 \%$ identity). Homologous genes were clustered and clusters containing a single gene copy in each of the genomes were selected. The resulting list of 898 putative single copy genes and their dedicated gene model assignments from the annotated $M$. circinelloides genome, with protein name taken from the corresponding $L$. corymbifera genome allowed the manual evaluation of suitable candidate markers. The criteria therefore were gene length (1000-3000 bp), intron frequency and length, and homology among the taxa under study. The evaluation of all 898 putative single copy genes was done manually using PhylomeDB (Huerta-Cepas et al. 2014) and JGI (Nordberg et al. 2014) (http://genome.jgi.doe.gov/ pages/search-for-genes.jsf?organism=Mucci2), with available tools for searching and visualization. Eight candidate genes complied with the criteria. Degenerated primers were designed using alignments of Mucorales available on JGI (January 2015). PCR performance was tested on a set of 26 strains covering the Mucoraceae, the Backusellaceae, the Choanephoraceae, the Mycotyphaceae, the Pilobolaceae, and the Rhizopodaceae. Products of primer pairs with high amplification rates (at least two thirds of the test set) were sequenced and phylogenetic trees were calculated.

\section{Sequence analyses}

Editing of the sequences and construction of consensus sequences was done using SeqMan v. 11.0.0 (DNASTAR, Lasergene). Sequences were deposited in GenBank (Table 1). Se-

Table 2 Primers used for amplification and sequencing

\begin{tabular}{|c|c|c|c|c|}
\hline Gene & Primers & Sequence 3'-5' & Reference & $\begin{array}{l}\text { Annealing } \\
\text { temperature }\end{array}$ \\
\hline$c f s$ & $\begin{array}{l}\text { CFS-f1 } \\
\text { CFS-f2 } \\
\text { CFS-r1 }\end{array}$ & $\begin{array}{l}\text { TTY TCY CGI TTY GCT CCT CGT } \\
\text { TTY TCY CGI TTY GCY CCT CG } \\
\text { ACC ARA TRAART CYT CRT ATT GCC A }\end{array}$ & $\begin{array}{l}\text { This study } \\
\text { This study } \\
\text { This study }\end{array}$ & $\begin{array}{l}54-55^{\circ} \mathrm{C} \\
54-55^{\circ} \mathrm{C} \\
54-55^{\circ} \mathrm{C}\end{array}$ \\
\hline ITS & $\begin{array}{l}\text { ITS1 (forward) } \\
\text { ITS4 (reverse) } \\
\text { V9G (forward) } \\
\text { LR3 (reverse) }\end{array}$ & $\begin{array}{l}\text { TCC GTA GGT GAA CCT GCG G } \\
\text { TCC TCC GCT TAT TGA TAT GC } \\
\text { TTA CGT CCC TGC CCT TTG TA } \\
\text { CCG TGT TTC AAG ACG GG }\end{array}$ & $\begin{array}{l}\text { White et al. (1990) } \\
\text { White et al. (1990) } \\
\text { De Hoog \& Gerrits van den Ende (1998) } \\
\text { Vigalys \& Hester (1990) }\end{array}$ & $\begin{array}{l}52{ }^{\circ} \mathrm{C} \\
52{ }^{\circ} \mathrm{C} \\
52{ }^{\circ} \mathrm{C} \\
52{ }^{\circ} \mathrm{C}\end{array}$ \\
\hline $\mathrm{mcm} 7$ & $\begin{array}{l}\text { Mcm7-709f } \\
\text { Mcm7-1348rev }\end{array}$ & $\begin{array}{l}\text { ACI MGI GTI TCV GAY GTH AAR CC } \\
\text { GAY TTD GCI ACI CCI GGR TCW CCC AT }\end{array}$ & $\begin{array}{l}\text { Schmitt et al. (2009) } \\
\text { Schmitt et al. (2009) }\end{array}$ & $\begin{array}{l}54-56^{\circ} \mathrm{C} \\
54-56^{\circ} \mathrm{C}\end{array}$ \\
\hline$r p b 1$ & $\begin{array}{l}\text { RPB1-f1 } \\
\text { RPB1-F1843 } \\
\text { RPB1-R3096 }\end{array}$ & $\begin{array}{l}\text { GTS CAR GAY ACI CTY TGT GG } \\
\text { ATT TYG AYG GTG AYG ARA TGAAC } \\
\text { GRA CRG TDC CRT CAT AYT TRA CC }\end{array}$ & $\begin{array}{l}\text { This study } \\
\text { Houbraken \& Samson (2011) } \\
\text { Houbraken \& Samson (2011) }\end{array}$ & $\begin{array}{l}52^{\circ} \mathrm{C} \\
52^{\circ} \mathrm{C} \\
52{ }^{\circ} \mathrm{C}\end{array}$ \\
\hline tsr1 & $\begin{array}{l}\text { TSR1-f2 } \\
\text { Tsr1-f1 } \\
\text { Tsr1-r2 }\end{array}$ & $\begin{array}{l}\text { TTY AGA ACI TCI RCY TGG GA } \\
\text { TTY CCN GAY GAR ATH GAY AC } \\
\text { TTC ATR TAI CCR TGI GTI CC }\end{array}$ & $\begin{array}{l}\text { This study } \\
\text { Hermet et al. (2012) } \\
\text { Hermet et al. (2012) }\end{array}$ & $\begin{array}{l}53.5^{\circ} \mathrm{C} \\
52^{\circ} \mathrm{C} \\
52{ }^{\circ} \mathrm{C}\end{array}$ \\
\hline
\end{tabular}


quences were aligned using MAFFT v. 7 (Katoh et al. 2017) with default settings followed by slight manual corrections. For each marker phylogenetic trees were calculated using RAxML-HPC BlackBox provided on Cipres Science Gateway v. 3.3 (Miller et al. 2010) with default settings. The corresponding partition model was compiled using PartitionFinder2 (Lanfear et al. 2016) and maximum parsimony (MP) implemented in PAUP (v. 4.0a, default settings). The topology of all gene trees was checked manually for conflicts applying the principle of Kauff \& Lutzoni (2002), stating that a conflict is significant if a set of taxa is monophyletic in one gene genealogy and non-monophyletic in another gene with bootstrap supports of more than $70 \%$ in $\mathrm{ML}$ analyses. Concatenation of alignments was done in Geneious v. 11.0.3 (Biomatters, Auckland, New Zealand). Molecular phylogeny of the concatenated alignment was again assessed by RAxML and MP using default settings. Mucor racemosus f. racemosus, CBS 260.68 served as outgroup. Phylogenetic trees were illustrated by TreeGraph2 (Stöver \& Müller 2010) and Adobe Illustrator (v. 15.1.0, Abode Systems, San José, USA). Gene trees and concatenated trees are deposited at TreeBASE (http://purl.org/phylo/treebase/phylows/study/TB2:S23209).

\section{RESULTS}

\section{Phylogenetic markers in Mucorales}

To identify novel markers suitable for phylogenetic analyses in Mucor, we compared four mucoralean genomes. The CFS gene performed best regarding sequence variability and the rate of success in amplification and sequencing for the tested set of 26 Mucorales species. The gene model on JGI predicts a gene for a cyclopropane-fatty-acyl-phospholipid synthase of 1691 bp with two introns (https://genome.jgi.doe.gov/pages/ search-for-genes.jsf?organism=Mucci2, Protein ID: 175664). The primers are located in the first and third exon spanning both introns (Fig. 1). The resulting PCR product is about 880 bp long depending on the species.

Several markers that are commonly used in fungal phylogeny, such as the partial genes of actin, the translation elongation factor 1- $\alpha$ (tef), beta-tubulin and calmodulin were no single copy genes, judging from comparison of four mucoralean genomes. Direct sequencing of the four protein coding genes (cfs, $\mathrm{mcm} 7$, $r p b 1, t s r 1$ ) was successful, supporting their single copy status. The markers differ in degrees of variability: the highest number of parsimony informative sites was found in tsr1 (236), followed by rpb1 (173), cfs (157), mcm7 (137) and ITS (66).

\section{Phylogenetic analyses}

In order to reconstruct the molecular phylogenetic relationships in the MCC a total number of 383 DNA sequences belonging to 93 taxa were newly generated for this study (Table 1): cfs (91), mcm7 (90), rpb1 (91), tsr1 (92) and ITS (19). Only occasionally amplification of the gene of interest failed. In total the alignments comprised 3536 unambiguously aligned sites, i.e., $777 \mathrm{bp}$ for $c f s, 610 \mathrm{bp}$ for ITS, $522 \mathrm{bp}$ for $\mathrm{mcm} 7,799 \mathrm{bp}$ for $r p b 1$, and 828 bp for tsr1. RAxML and Maximum Parsimony (MP) analyses were done for individual genes and for the con- catenated dataset. Applying the principles of Kauff \& Lutzoni (2002), conflicts among gene topologies were detected only concerning two strains, $M$. bainieri CBS 293.63 and $M$. ramosissimus CBS 135.63, with strongly deviating sequences for all markers. Considering positions in clades with bootstrap support of $75 \%$ or higher, M. bainieri CBS 293.63 is either part of a clade including CBS 338.71, CBS 540.78, and CBS 543.80 (cfs/RAxML and ML, rpb1/ RAxML and ML) or of a clade consisting of $M$. circinelloides $\mathrm{f}$. lusitanicus, $M$. variicolumellatus and $M$. ramosissimus ( $m c m 7 / \mathrm{ML}$, tsr1/RAxML and ML). Mucor ramosissimus CBS 135.63 is positioned in supported clades with $M$. circinelloides $\mathrm{f}$. Iusitanicus, $M$. variicolumellatus and M. ramosissimus (cfs/ML, mcm7/RAxML and $\mathrm{ML}$, tsr1/RAxML and $\mathrm{ML}$ ) or with the strains CBS 338.71, CBS 540.78, and CBS 543.80, and M. bainieri CBS 293.63 (rpb1/ML). Despite these small conflicts, we decided to concatenate the five loci.

Phylogenetic species (PS) were recognised using the criteria of genealogical concordance and non-discordance (Dettman et al. 2003), which means that a clade was regarded as phylogenetic species if it is present in all phylogenetic trees independent of the level of support and/or if it had a high degree of support in at least one gene genealogy without contradictory support in another locus. Applying these criteria, we recognised 16 PS (Fig. 2). The formae circinelloides, griseocyanus, and janssenii contained two PS each. In all analyses, strain $M$. circinelloides f. circinelloides CBS 846.72A is positioned basal to the two PS of forma circinelloides. Forma lusitanicus represents a single PS. The second PS (PS 2) within forma janssenii is known as M. velutinosus (Álvarez et al. 2011). Mucor bainieri (PS 3) and $M$. ramosissimus (PS 12) are represented by single strains that clustered separately in all analyses and are consequently recognised as PS. The sporangiola-forming species Ellisomyces anomalus (PS 5) and Mucor ctenidus (syn. Backusella ctenidia) (PS 16) clearly belong in the MCC and are also recognised as PS.

Five PS were recognised by our analyses (Fig. 2) that do not match with known species: PS 4 (CBS 540.78 and CBS 543.80), PS 6 (CBS 202.28), PS 9 (CBS 526.68 and CBS 846.73), PS 11 (CBS 236.35 and JMRC:SF:012536), and PS 13 (CBS 541.78). In most phylogenetic trees, strains CBS 540.78 and CBS 543.80 (PS 4) form a well-supported clade with strains CBS 338.71 and CBS 635.65. However, in the genealogies of $c f s$ and $r p b 1$ M. bainieri CBS 293.63 (cfs/RAxML and MP) or M. bainieri CBS 293.63 and $M$. ramosissimus CBS 135.65 (rpb1/RAxML and MP) are part of the clade and form a supported subclade with CBS 338.71. Only the subclade of CBS 540.78 and CBS 543.80 presents in all analyses.

\section{Morphology}

Members of the $M$. racemosus group consisting of MCC and MRC are characterised by: sporangia formed on repeatedly sympodially branched sporangiophores (or sporangia absent and multispored pedicellate sporangiola present that develop on irregularly to sympodially branched sporophores), mostly two types of sporangiophores (short intensively sympodially branched sporangiophores and tall less branched sporangio-

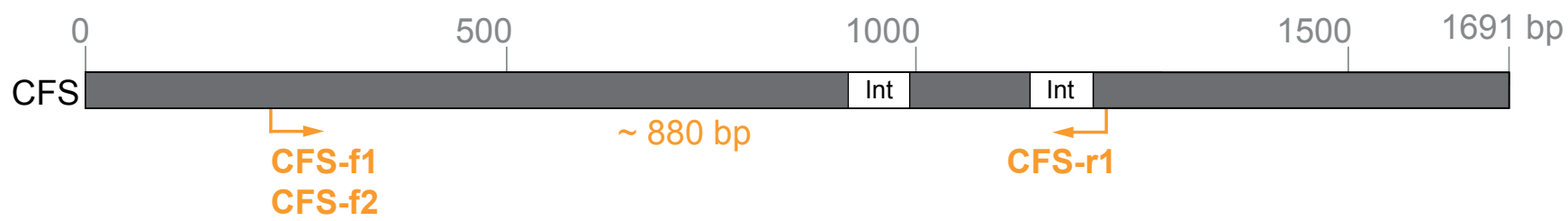

Fig. 1 Gene model of the putative cyclopropane-fatty-acyl-phospholipid synthase following the gene prediction (JGI, estExt_Genewise1Plus.C_011066 of Mucor circinelloides CBS277.49 genome v2.0) with primer binding sites and estimated amplicon length. 


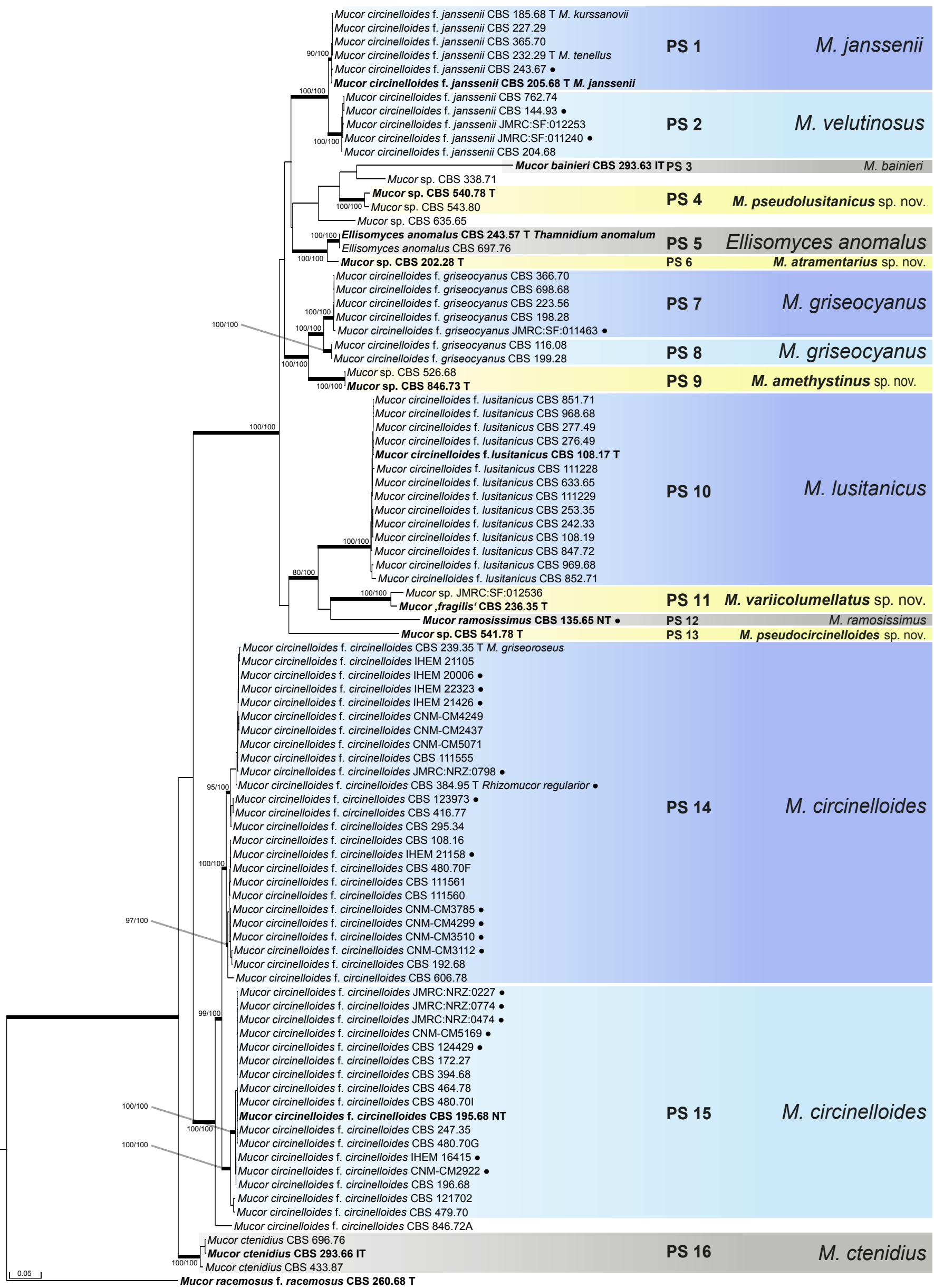

Fig. 2 Phylogenetic tree based on the concatenated alignment of five molecular markers (cfs, $m c m 7$, ITS, $r p b 1$, tsr1) calculated using RAxML. Branches with bootstrap values $\geq 75$ in the RAxML and/or the Maximum Parsimony analyses are printed in bold and the bootstrap values are given, in this order. $T=$ ex-type strain, IT = ex-isotype strain and NT = ex-neotype strain. Ex-type strains of currently accepted taxa are printed in bold. Clinical isolates are marked by black circles. 

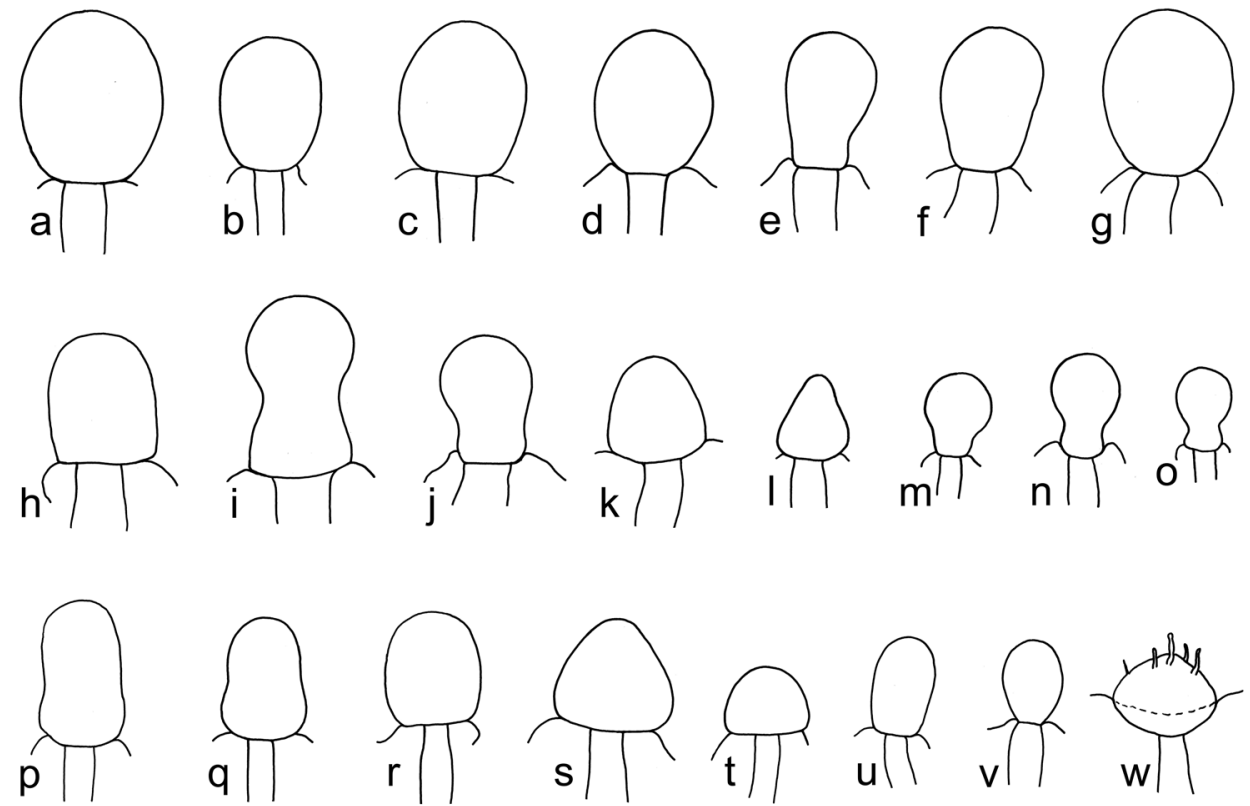

Fig. 3 Columellae of the M. racemosus group: a-g. MCC, typical columellae of tall sporangiophores without or only with slightly truncate bases (a-b. M. circinelloides CBS 195.68; c. M. circinelloides CBS 384.95; d-g. M. janssenii CBS 205.68); $\mathrm{h}-\mathrm{o}$. MCC columellae of short sporangiophores of $M$. janssenii and M. velutinosus ( $\mathrm{h}-\mathrm{I}$. M. velutinosus JMRC:SF:011240; $\mathrm{m}-\mathrm{o}$. M. janssenii CBS 185.68); $\mathrm{p}-\mathrm{w}$. MRC, typical columellae ( $\mathrm{p}-\mathrm{q}$. M. racemosus f. racemosus CBS 260.68; r-t. M. racemosus f. racemosus CBS 774.86; u-v. M. racemosus f. sphaerosporus CBS 115.08; w. M. plumbeus CBS 226.36).
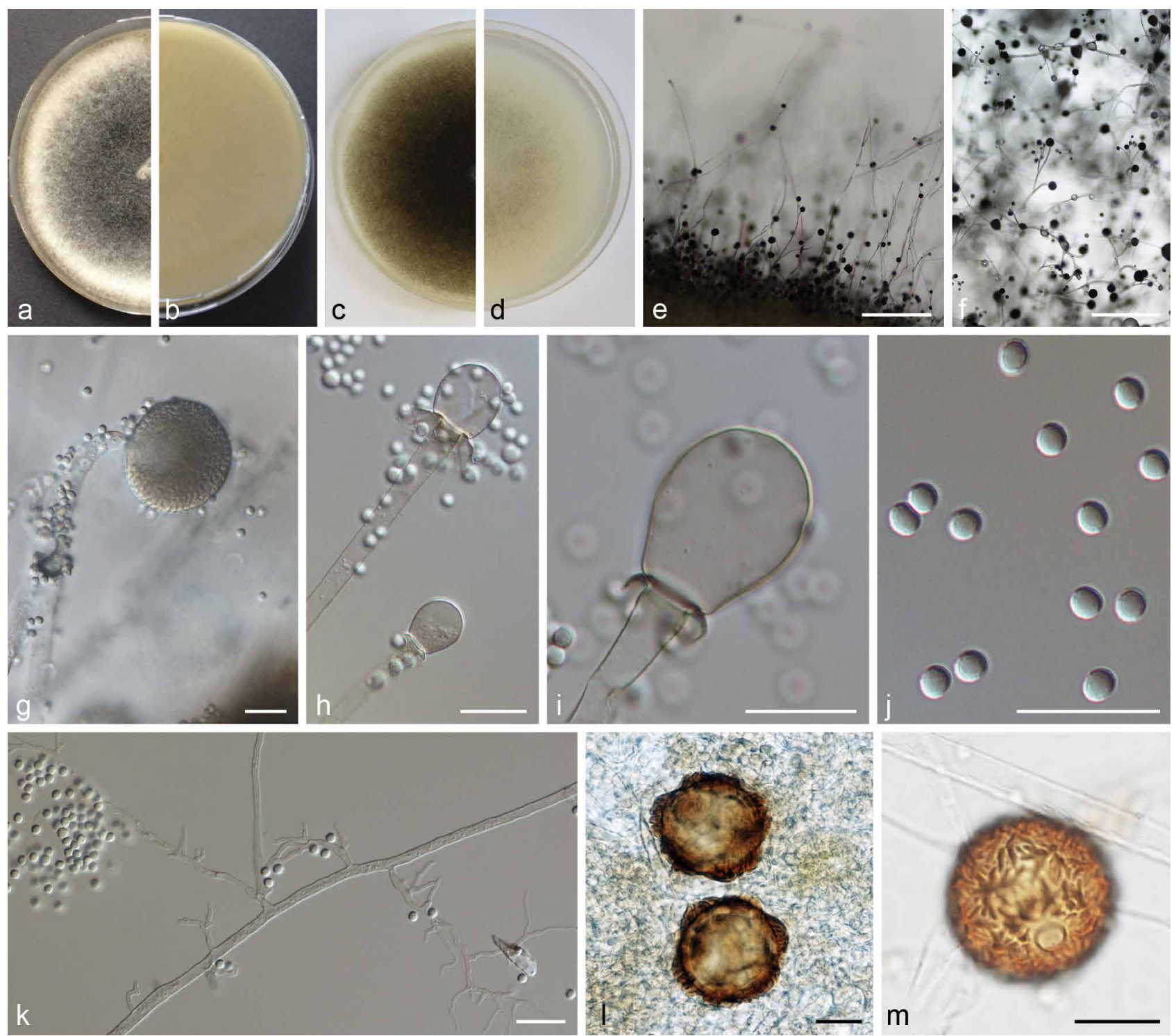

Fig. 4 PS 1 (M. janssenii) CBS 205.68. a. Front and b. reverse of a 7-d-old culture on MEA at room temperature (RT); c. front on PDA; d. front on SMA; e. lateral view; f. top view; g. sporangium; h-i. columellae with collar; j. sporangiospores; k. rhizoids; I-m. zygospores of crosses with $M$. griseocyanus CBS 116.08. - Scale bars: $e-f=500 \mu m ; g-m=20 \mu m$. 

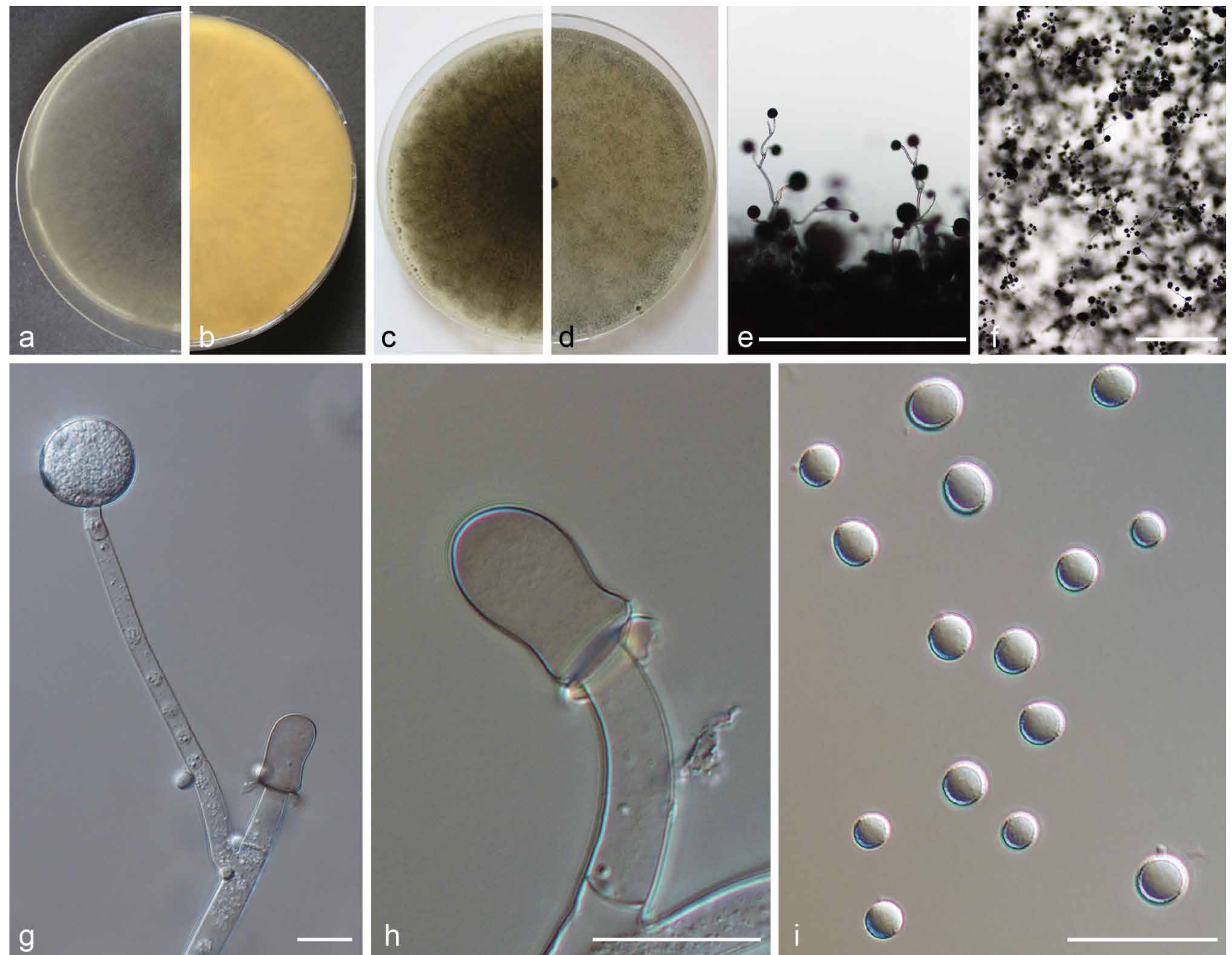

Fig. 5 PS 2 (M. velutinosus) CBS 762.74. a. Front and b. reverse of a 7-d-old culture on MEA at RT; c. front on PDA; d. front on SMA; e. lateral view; f. top view; g. sporangiophore with sporangium and columella; h. columella with collar; i. sporangiospores. - Scale bars: $e-f=500 \mu \mathrm{m} ; g-i=20 \mu \mathrm{m}$.

phores), tips of the sporangiophores that usually form a sporangium, rather small sporangia (on MEA usually less than $100 \mu \mathrm{m}$ diam except of $M$. amethystinus with sporangia with diam of up to $160 \mu \mathrm{m})$, sporangiospores either subglobose and less than $13 \mu \mathrm{m}$ diam or ellipsoidal and less than $10 \mu \mathrm{m}$ in length (except of the irregularly shaped sporangiospores of $M$. lusitanicus), and red-brown to dark brown (but not black) zygospores with a maximal diam of 25 to $80 \mu \mathrm{m}$ on WA (measured without ornamentation). Members of the MCC differ from MRC by slender sporangiospores. Members of the MRC usually have distinctly truncated columellae (Fig. 3p-w) while MCC members have non-truncated columellae (Fig. $3 a-g$ ) except of $M$. janssenii and $M$. velutinosus (Fig. $3 \mathrm{~h}-\mathrm{o}$ ).

On the basis of the above phylogenetic analyses (Fig. 2), we searched for morphological differences between phylogenetic species applying the following characters: 1) presence of two types of sporangiophores (type 1 - small and sympodially branched in a short distance, type 2 - tall and loosely sympodially branched); 2) colour and size of the sporangia; 3 ) height of the mycelium; 4) branching and diameter of sporangiophores; 5) size and shape of columellae; 6 ) size, shape, length/width ratio and ornamentation of sporangiospores, and presence of: 7) chlamydospores; and 8) rhizoids. Sporangia-forming species of the MCC usually form two types of sporangiophores although strains differ concerning the distinctness of this feature. Mucor bainieri (PS 3) forms azygophores instead of small sporangiophores. PS 2 only forms the small type of sporangiophores.

The two PS of forma janssenii (PS 1 and PS 2) differ clearly in their morphology: PS 1 (Fig. 4) containing the ex-type strain of $M$. janssenii CBS 205.68 produces somewhat higher (up to $4 \mathrm{~mm}$ ), cottony to slightly funiculose mycelia consisting of small and somewhat taller sporangiophores, predominantly obovoid columellae and smaller sporangiospores (up to 6 , rarely up to $7 \mu \mathrm{m}$ diam). In contrast, the mycelia of PS 2 (M. velutinosus) (Fig. 5) are flat (only up to $2 \mathrm{~mm}$ in height), velutinous and consist mainly of the small type of sporangiophores, although a few longer sporangiophores may develop. In most strains the majority of columellae is keyhole-shaped (Fig. $5 \mathrm{~h}$ ), but also obovoid columellae occur. The sporangiospores are up to 9 (rarely 12) $\mu \mathrm{m}$ diam. Strain CBS 204.68 developed a higher proportion of larger sporangiophores and consequently it had a more funiculose appearance. In the case of the two PS detected in forma circinelloides (PS 14 and PS 15; Fig. 6) and in forma griseocyanus (PS 7 and PS 8; Fig. 7) no differentiating morphological characters were found.

Mucor bainieri (PS 3; Fig. 8) is characterised by the formation of azygospores on branched azygophores. PS 4 (Fig. 9) is morphologically similar to forma lusitanicus (PS 10; Fig. 10) because it has brown-grey sporangia, predominantly globose columellae and ellipsoidal sporangiospores. However, it differs from forma lusitanicus by a lower Tmax $\left(35^{\circ} \mathrm{C}\right.$ compared to $37-38^{\circ} \mathrm{C}$ ) and by a different appearance of the mycelium: in PS 4 sporangiospores of sporangia that touch the surface of the medium tend to germinate with short sporangiophores and to produce dense patches within the mycelium that we designate as protuberances (Fig. $9 \mathrm{~g}$ ). Strains CBS 338.71 and CBS 635.65 differ only slightly from CBS 540.78 and CBS 543.80: CBS 338.71 has a higher growth speed reaching a diam of $9 \mathrm{~cm}$ 

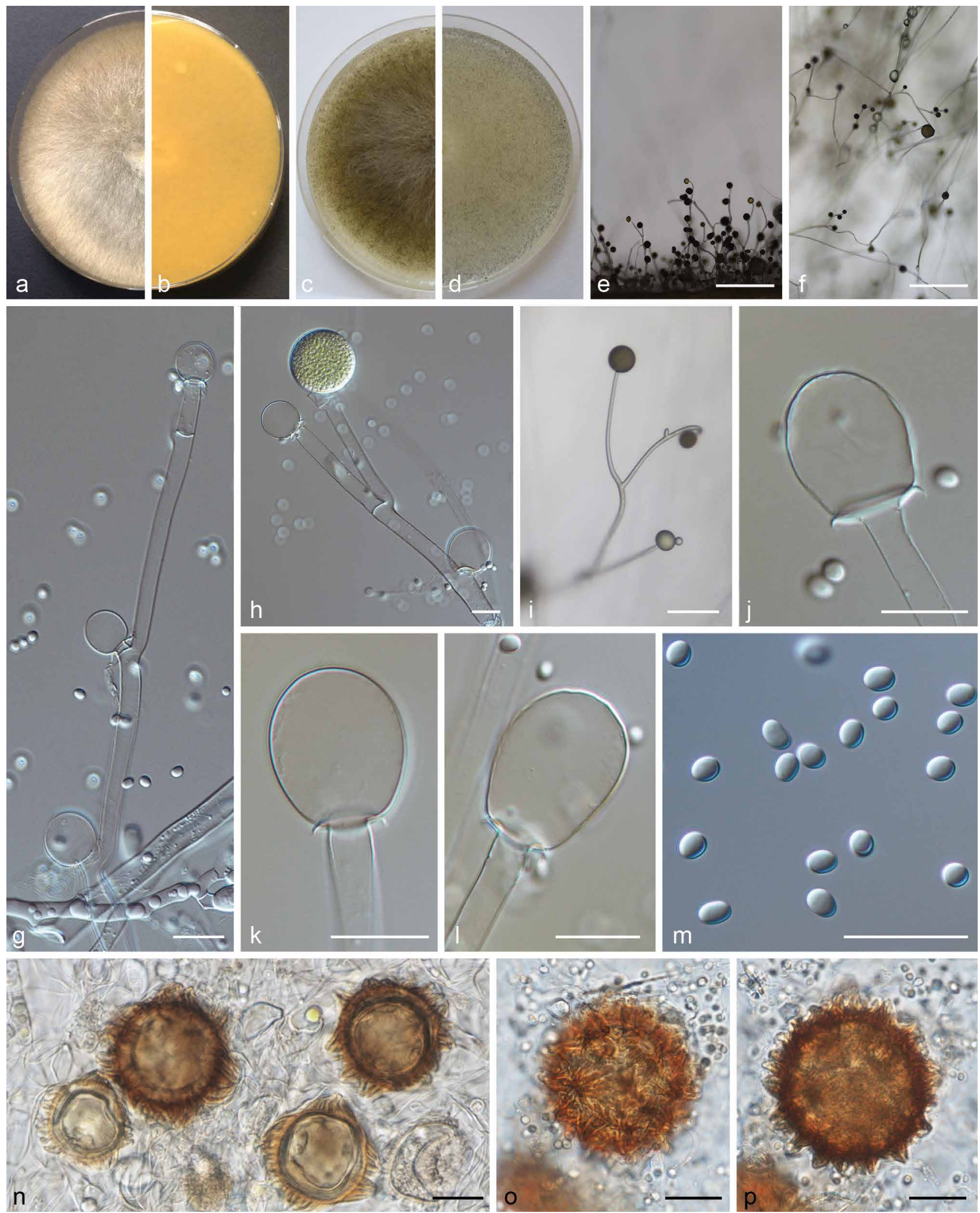

Fig. 6 PS 14/15 (M. circinelloides) (a-h, k-m. CBS 195.68; i-j. CBS 384.95). a. Front and b. reverse of a 7-d-old culture on MEA at RT; c. front on PDA; d. front on SMA; e. lateral view; f. top view; g. sporangiophore with columellae; h. sporangiophore with sporangium and columella; i. sporangiophore with sporangia; j-I. columella; $m$. sporangiospores; $n$. zygospore formed in crosses of CBS $192.68 \times$ CBS 124429; o-p. zygospores formed in crosses of CBS 192.68 $\times$ CBS 394.68. - Scale bars: $e-f=500 \mu \mathrm{m} ; g-p=20 \mu \mathrm{m}$.

within $2 \mathrm{~d}$ and its maximum growth temperature is $36^{\circ} \mathrm{C}$. Strain CBS 635.65 differs from PS 4 by having a pale orange reverse, by lacking protuberances, by a maximum growth temperature of $33^{\circ} \mathrm{C}$, and by producing a small amount of slightly elongated columellae (Fig. 9l-m).

Ellisomyces anomalus (PS 5) has a strongly deviating morphology. It lacks sporangia and forms multispored sporangiola that develop on sympodially branched sporophores (Fig. 11, 12b).
The branches of the sporophores are swollen. The sporangiola of this species have well-developed columellae. In contrast to the sessile sporangia of the sympodially branched sporangiophores of M. ramosissimus (Fig. 12a) and the sporophores of $M$. ctenidius (Fig. 12c-d), no septa are formed beneath the sporangiola.

PS 6 (Fig. 13) can be distinguished from remaining PS of the MCC by its small, globose sporangiospores $(<5 \mu \mathrm{m}$ diam) 

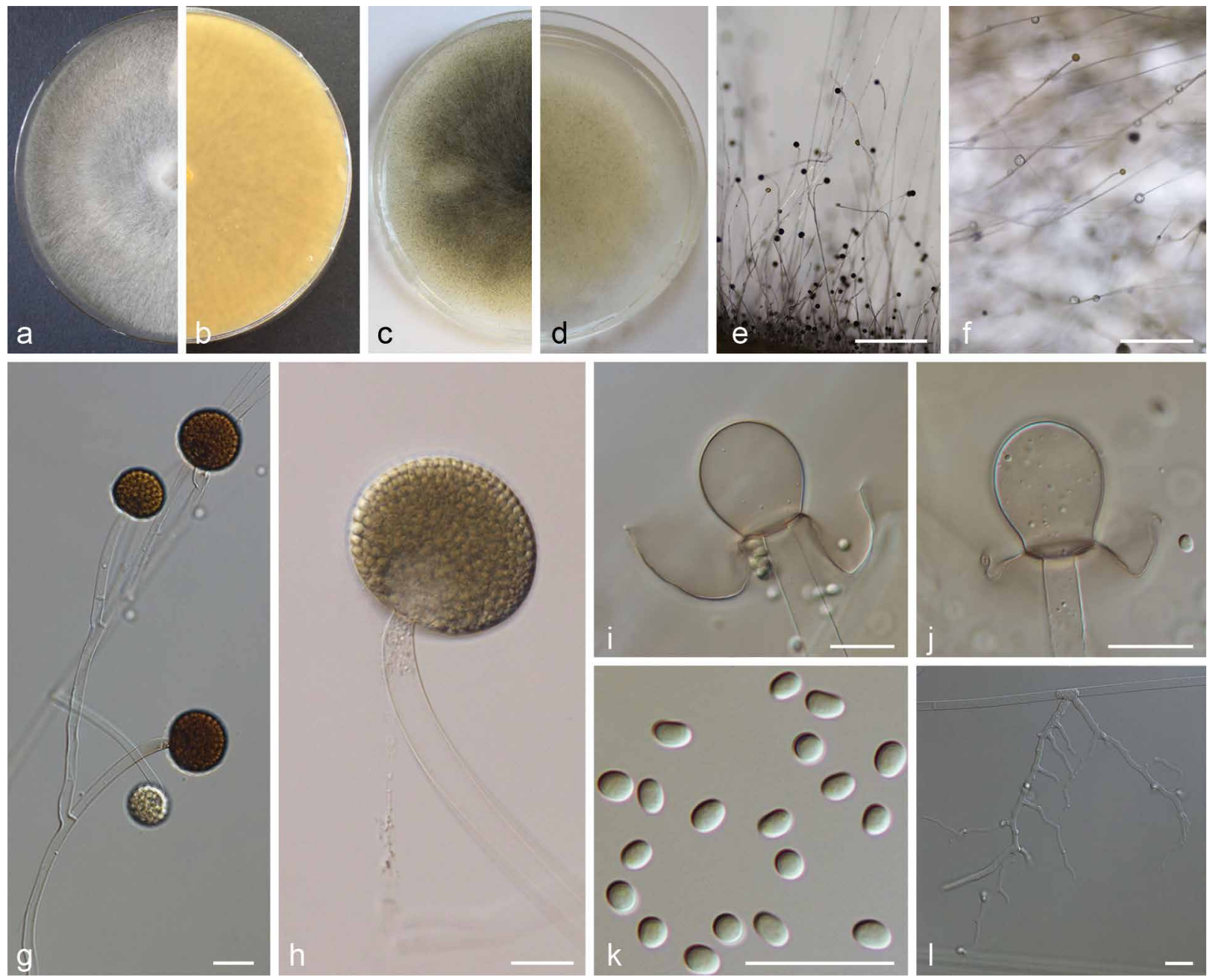

Fig. 7 PS 7 (M. griseocyanus) CBS 366.70. a. Front and b. reverse of a 7-d-old culture on MEA at R; c. front on PDA; d. front on SMA; e. lateral view; f. top view; g. sporangiophore with sporangia; h. sporangium; i-j. columella with collar; k. sporangiospores; I. rhizoids. - Scale bars: $e-f=500 \mu m ; g-I=20 \mu m$.
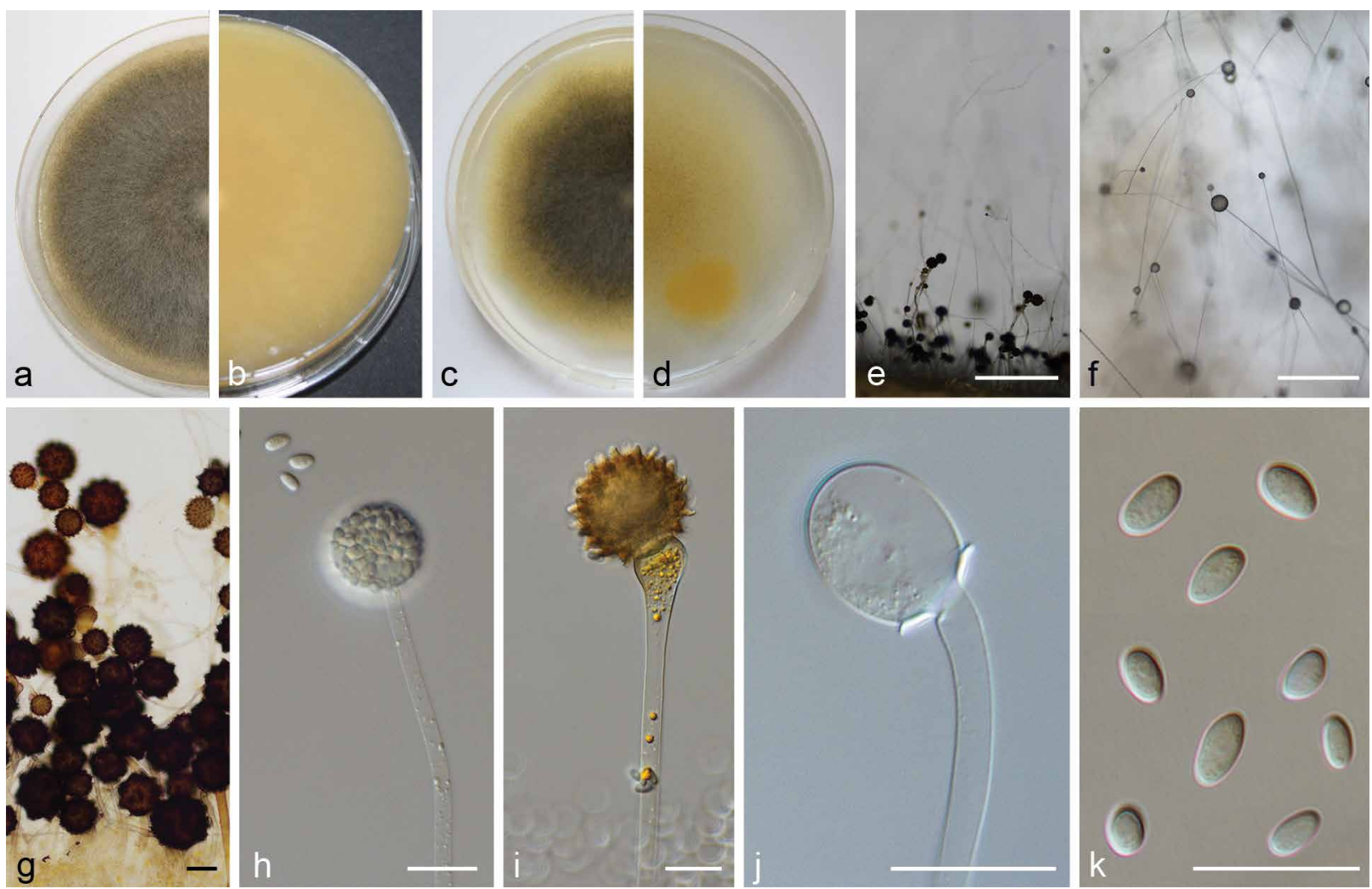

Fig. 8 PS 3 (M. bainieri) CBS 293.63. a. Front and b. reverse of a 7-d-old culture on MEA at RT; c. front on PDA; d. front on SMA; e. lateral view; f. top view; g. lateral view with azygospores; h. sporangium; i. azygospore; j. columella with collar; k. sporangiospores. - Scale bars: $e-f=500 \mu m ; g-k=20 \mu m$. 

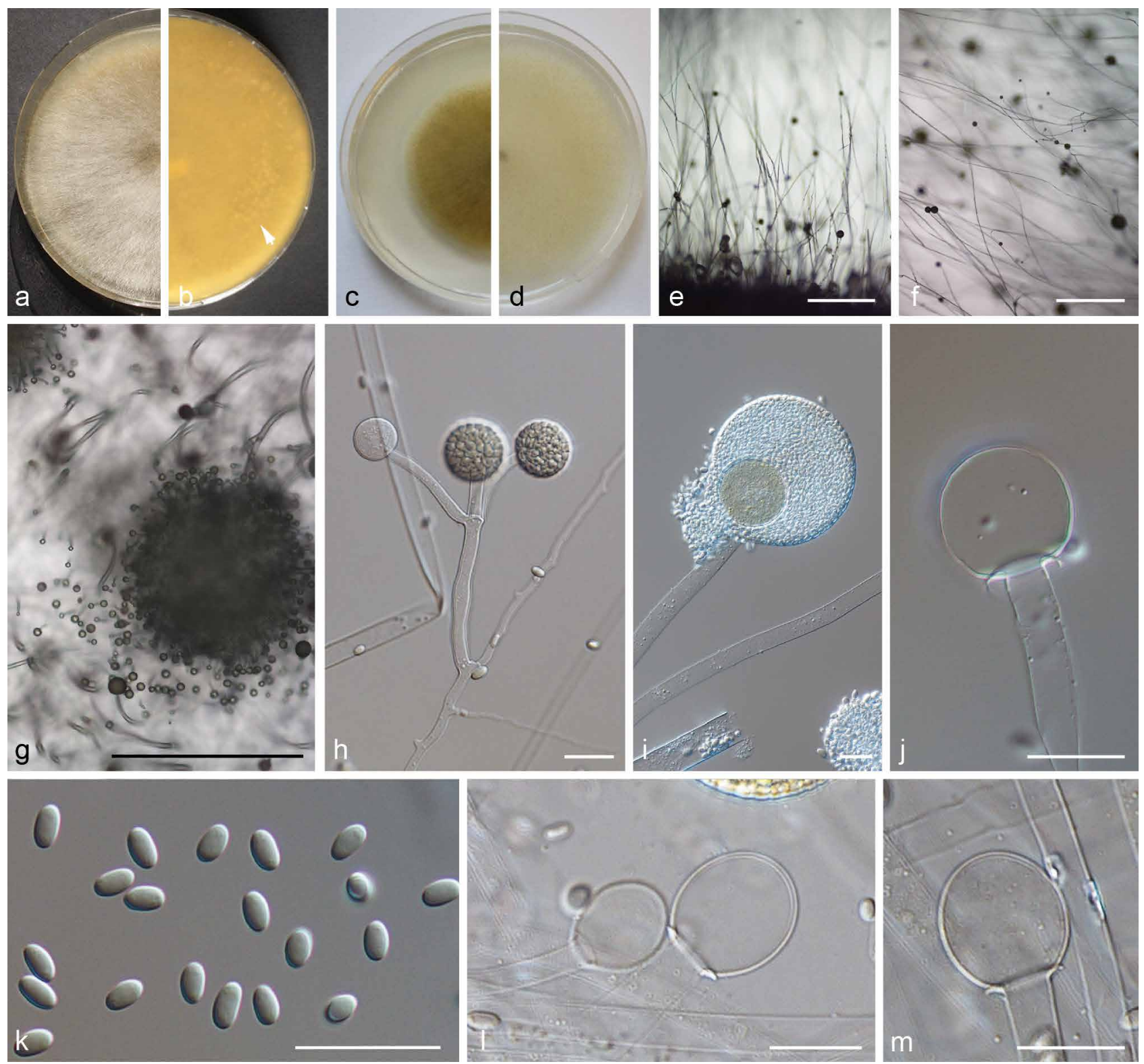

Fig. 9 PS 4 (M. pseudolusitanicus sp. nov.) CBS 540.78. a. Front and b. reverse with protuberances (arrow) of a 7-d-old culture on MEA at RT; c. front on PDA; d. front on SMA; e. lateral view; f. top view; g. top view on protuberance; h. sporangiophore with sporangia; i. sporangium; j. columella; $k$. sporangiospores; I-m. columellae of CBS 635.35. - Scale bars: $e-f=500 \mu \mathrm{m} ; \mathrm{g}-\mathrm{m}=20 \mu \mathrm{m}$.

Table 3 Maximum growth temperature of MCC and MRC species sorted in descending order.

Environmental/clinical strains

Maximum growth temperature

Number of strains examined

Mucor circinelloides (PS 14)

Mucor circinelloides (PS 15)

Mucor velutinosus (PS 2)

Mucor janssenii (PS 1)

Mucor lusitanicus (PS 10)

Mucor pseudocircinelloides (PS 13)

Mucor variicolumellatus (PS 11)

Mucor amethystinus (PS 9)

Mucor ctenidius (PS 16)

Mucor ramosissimus (PS 12)

Mucor pseudolusitanicus (PS 4)

Mucor griseocyanus (PS 7)

Mucor griseocyanus (PS 8)

Ellisomyces anomalus (PS 5)

Mucor atramentarius (PS 6)

Mucor bainieri (PS 3)

Mucor racemosus f. racemosus

Mucor plumbeus

Mucor racemosus f. sphaerosporus

Mucor brunneogriseus

$38-39 / 38-39{ }^{\circ} \mathrm{C}$
$37-38 / 38-39^{\circ} \mathrm{C}$
$36-38 / 37^{\circ} \mathrm{C}$
$34-38 / 38^{\circ} \mathrm{C}$
$35-37^{\circ} \mathrm{C}$
$37^{\circ} \mathrm{C}$
$36^{\circ} \mathrm{C}$
$36^{\circ} \mathrm{C}$
$35-36{ }^{\circ} \mathrm{C}$
$35^{\circ} \mathrm{C}$
$35^{\circ} \mathrm{C}$
$34-35{ }^{\circ} \mathrm{C}$
$35^{\circ} \mathrm{C}$
$34^{\circ} \mathrm{C}$
$34^{\circ} \mathrm{C}$
$34^{\circ} \mathrm{C}$
$31-34{ }^{\circ} \mathrm{C}$
$30^{\circ} \mathrm{C}$
$28^{\circ} \mathrm{C}$
$27^{\circ} \mathrm{C}$



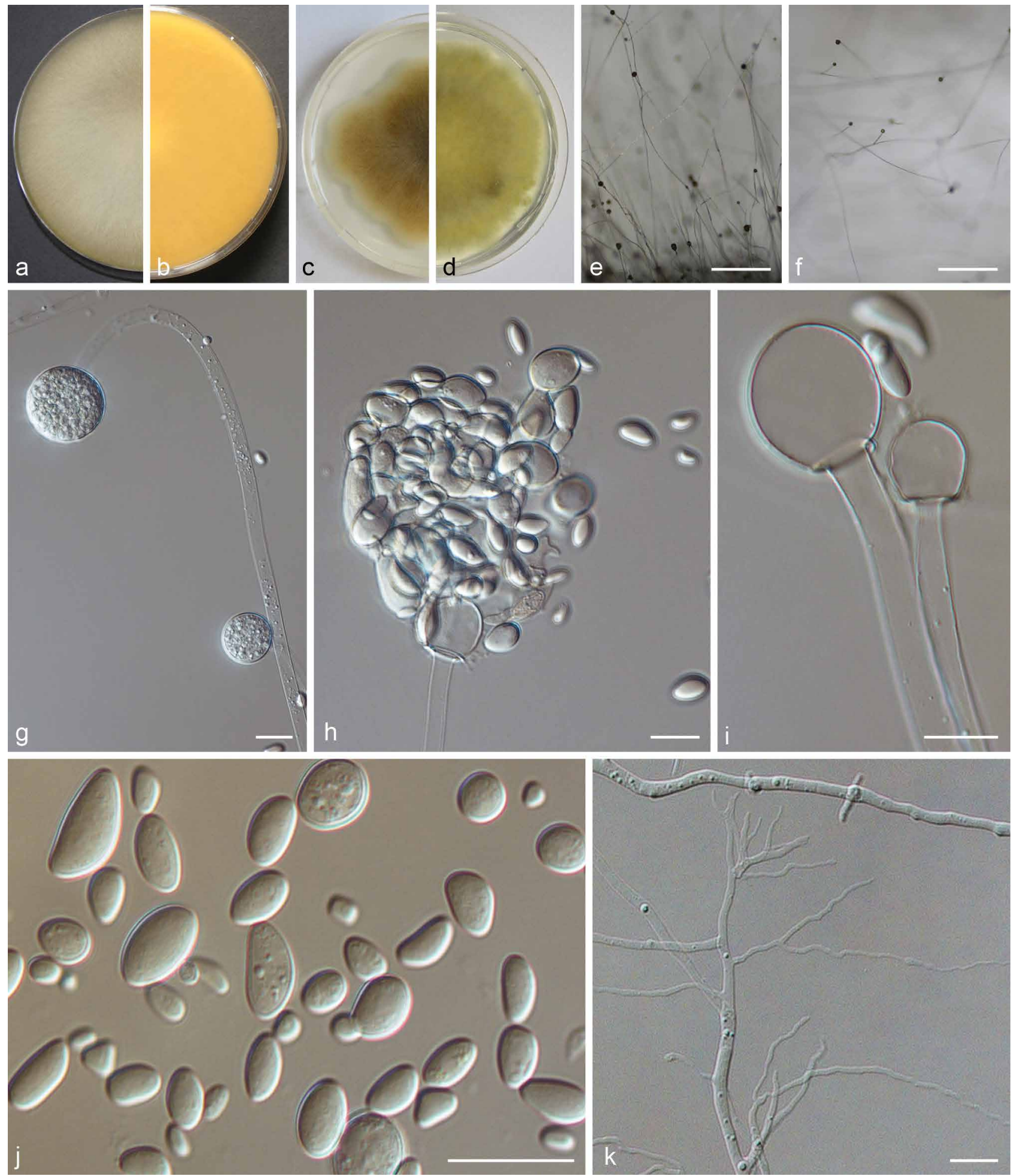

Fig. 10 PS 10 (M. lusitanicus) CBS 108.17. a. Front and b. reverse of a 7-d-old culture on MEA at RT; c. front on PDA; d. front on SMA; e. lateral view; f. top view; g. sporangiophore with sporangia; $h$. burst sporangium releasing sporangiospores; i. columellae; j. sporangiospores; k. rhizoids. - Scale bars: $e-f=500 \mu \mathrm{m} ; g-k=20 \mu \mathrm{m}$.

and its dark brown sporangia. Typical for PS 9 (Fig. 14) is the combination of predominantly globose columellae and globose sporangiospores, as well as the violaceus tinge of the mycelia especially in the peripheral area. PS 11 (Fig. 15) is similar to forma lusitanicus (PS 10; Fig. 10), but it forms not only globose columellae as $\mathrm{f}$. lusitanicus but also ellipsoidal (Fig. 15h), strawberry-shaped (Fig. 15i) or obovoid (Fig. 15I-m) columellae. Typical for M. ramosissimus (PS 12; Fig. 16) are its flat colonies, the often partially swollen sporangiophores, the predominantly applanate columellae, and the slightly angular sporangiospores (Fig. 16h).
PS 13 (Fig. 17) differs only slightly from forma circinelloides (Fig. 6). In contrast to f. circinelloides (PS 14 and PS 15) its sporangia are pale coloured and become light brown only if they dry out. The sporangiospores are slenderer $(5.2-8.6 \times$ 2.6-3.9 $\mu \mathrm{m}$ with a mean length/width ratio of $1.9(1.7-2.3)$ compared to those of $M$. circinelloides (PS 14 and PS 15; 3.6-9.9 $\times 2.1-5.8 \mu \mathrm{m}$ with a mean length/width ratio of 1.6 (1.5-1.8)). Mucor ctenidius (PS 16, Fig. 12c-d, 18) can be distinguished easily by the few-spored sporangiola formed in addition to terminal sporangia laterally on the sporangiophores. There are differences among the $M$. ctenidius strains 

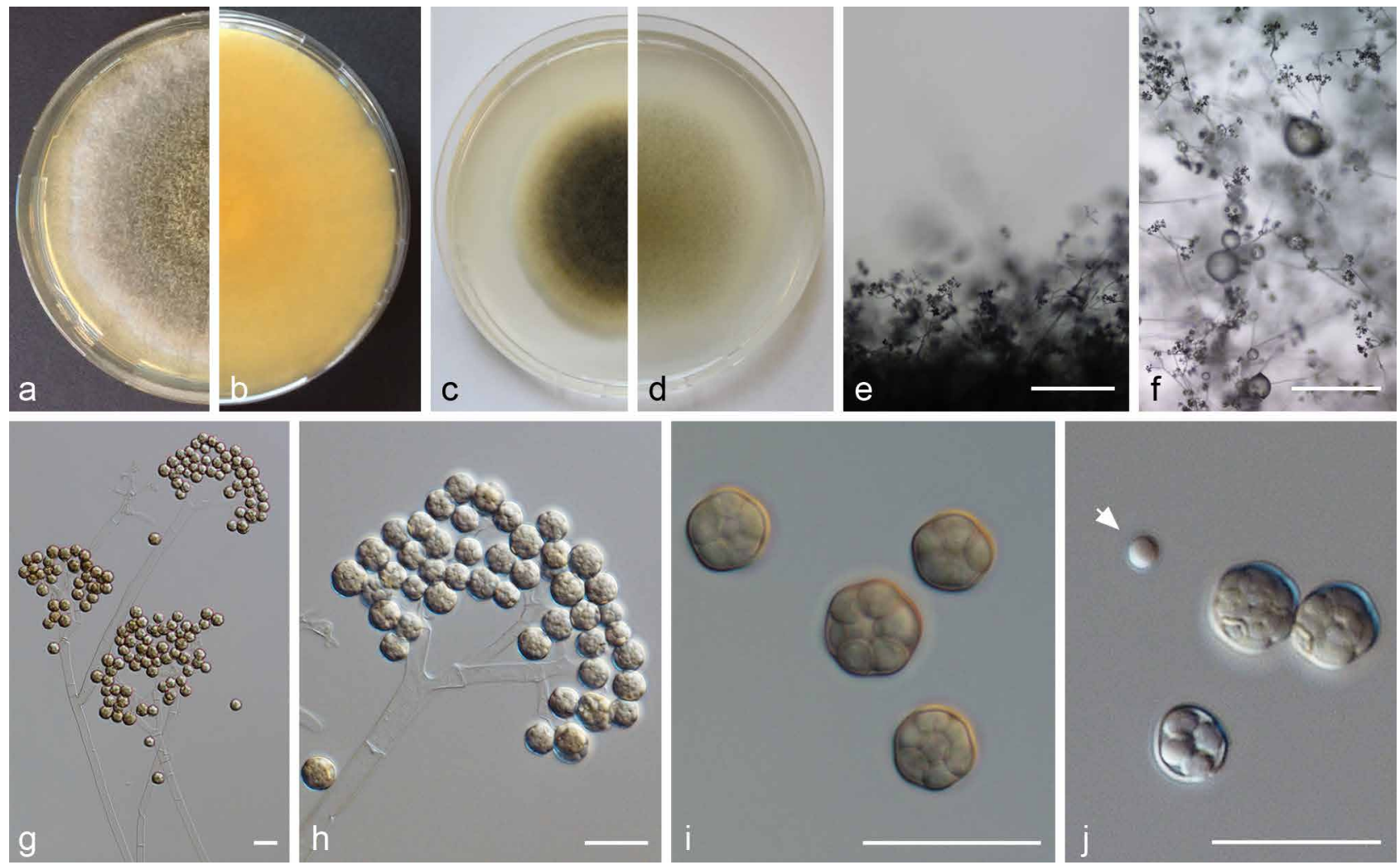

Fig. 11 PS 5 (Ellisomyces anomalus) CBS 243.57. a. Front and b. reverse of a 7-d-old culture on MEA at RT; c. front on PDA; d. front on SMA; e. lateral view; f. top view; g. sporophore with sporangiola; $h$. sporangiola on sporophore; i. sporangiola; $j$. sporangiola and single spore $($ arrow $)-$ Scale bars: $e-f=$ $500 \mu \mathrm{m} ; \mathrm{g}-\mathrm{j}=20 \mu \mathrm{m}$.

concerning the sporangiola bearing hyphae: CBS 293.66 has predominantly unbranched sporangiola-bearing hyphae (Fig. 12d), while they arise in clusters in CBS 433.87 (Fig. 12c). Some hyphae possess basal septae which suggests sympodial branching (Fig. 12c-d).

Nearly all species of the MCC grow as yeasts in liquid YPG medium with a glucose content of $3 \%$. Only $M$. atramentarius and $M$. ctenidius showed hyphal growth under this condition. Morphological features of the yeast cells are similar among the members of the MCC and cannot be used to distinguish species.
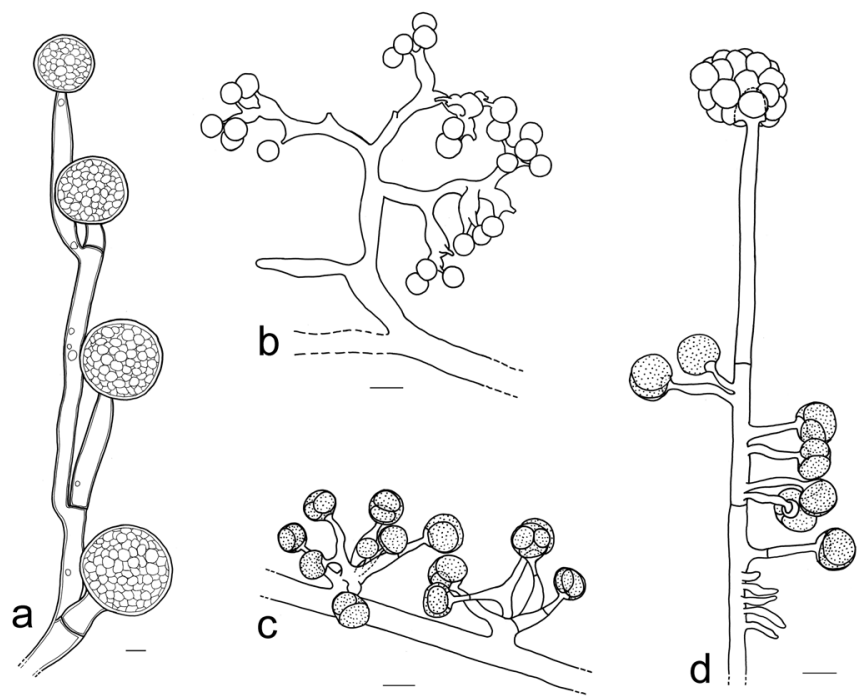

Fig. 12 Sporophores in the MCC. a. M. ramosissimus CBS 135.65; b. Ellisomyces anomalus CBS 243.37 (young sporophore); c. M. ctenidius CBS 433.87; d. M. ctenidius CBS 293.66. - Scale bars $=10 \mu \mathrm{m}$.

\section{Maximum growth temperature}

The maximum growth temperatures (Tmax) of the MCC listed in Table 3 range from 34 to $39{ }^{\circ} \mathrm{C}$ and differ among the PS. There are no differences of Tmax between PS of the same formae: PS 14 and PS 15 of $f$. circinelloides and PS 7 and PS 8 of f. griseocyanus. The highest Tmax in the set of strains analysed of $39^{\circ} \mathrm{C}$ is reached by PS 14 and 15 (f. circinelloides), followed by PS 2 ( $M$. velutinosus, $38^{\circ} \mathrm{C}$ ) and PS 1 ( $M$. janssenii, $\left.38^{\circ} \mathrm{C}\right)$. On average, clinical isolates do not have higher Tmax than environmental strains (Table 3 ). Members of the MRC have lower Tmax ranging from of 27 to $34{ }^{\circ} \mathrm{C}$ (Table 3 ).

\section{Zygospore formation}

Mating tests were performed in a total of 201 pairs of 50 strains (Table S1 and S2), comprising 52 mating tests between two strains of the same PS (intra PS crosses) and 149 mating tests between different PS (inter PS crosses). Based on current taxonomy, the total number of 201 mating tests consisted of 76 mating tests within the same forma (intraformae crosses), 69 mating tests between different formae (interformae crosses) and 56 confrontations between strains not assigned to a forma.

Zygospores were detected after 10-28 d at room temperature in the dark. The medium influences size and ornamentation of zygospores: they sometimes were larger when formed on WA than on MEA (e.g., CBS $242.33 \times$ CBS 852.71: mean diam (in all cases measured without ornamentation) $57 \mu \mathrm{m}$ (WA) vs $48 \mu \mathrm{m}$ (MEA), mean height of ornamentation $10.2 \mu \mathrm{m}$ (WA) vs $7.5 \mu \mathrm{m}$ (MEA); CBS $394.68 \times$ CBS 192.68: mean diam $54 \mu \mathrm{m}$ (WA) vs $47 \mu \mathrm{m}$ (MEA), mean height of ornamentation $12.0 \mu \mathrm{m}$ (WA) vs $9.2 \mu \mathrm{m}(\mathrm{MEA}))$. In one case (CBS $852.71 \times$ CBS 277.49) we found zygospores only on MEA. However, preliminary experiments showed that more positive pairings were obtained on WA than on MEA, and consequently further mating tests were performed on WA only. 

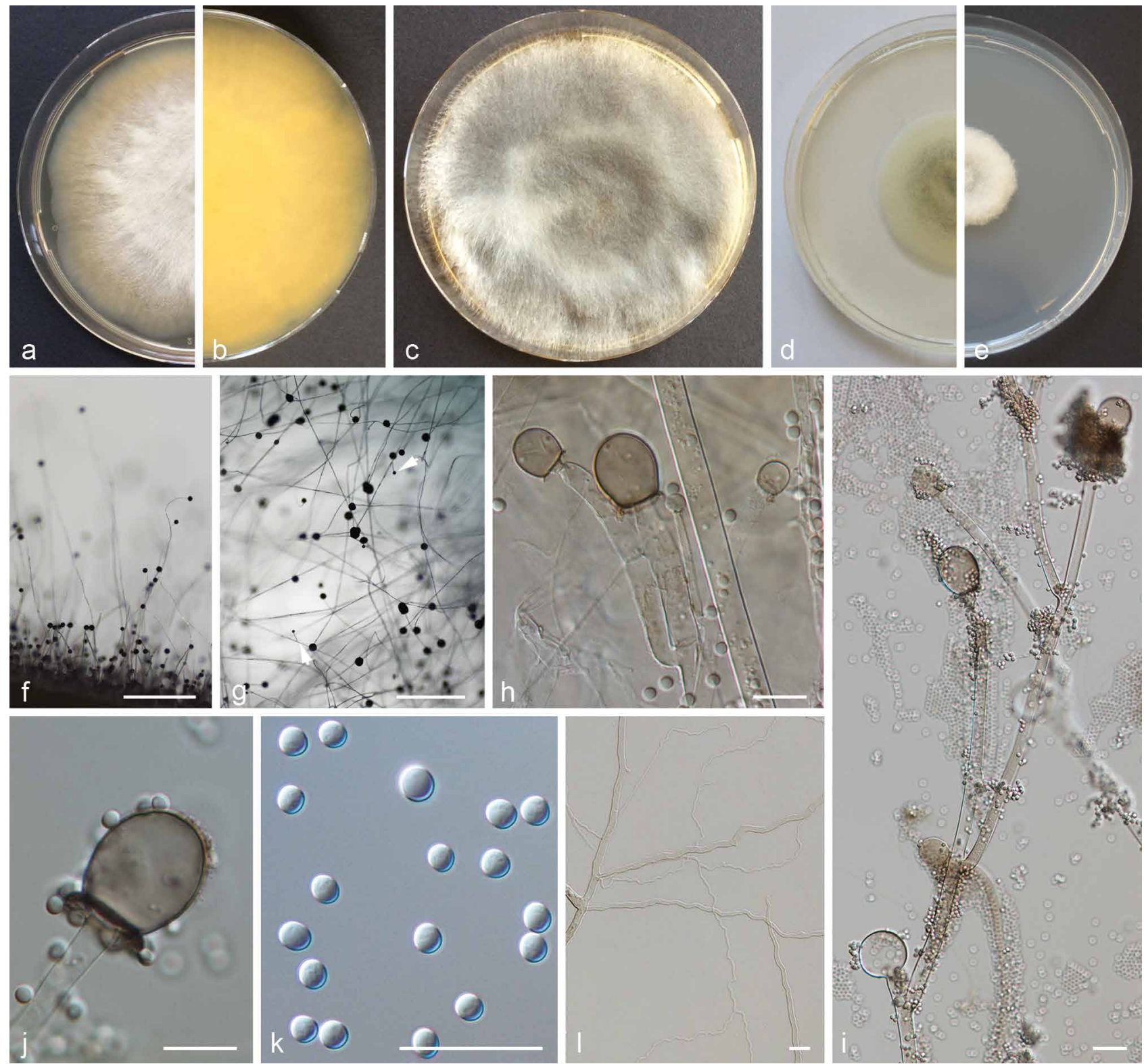

Fig. 13 PS 6 (M. atramentarius sp. nov.) CBS 202.28. a. Front and b. reverse of a 7-d-old culture on MEA at RT; c. front view of a 3 weeks old culture on MEA; d. 7-d-old culture on PDA and e. front on SMA; f. lateral view; g. top view; h. sporangiophore and columellae; i. sporangiophore; j. columella; k. sporangiospores; I. rhizoids. - Scale bars: $f-g=500 \mu \mathrm{m} ; \mathrm{h}-\mathrm{I}=20 \mu \mathrm{m}$.

Zygospores of the MCC were orange-brown to dark brown, 25-78 $\mu \mathrm{m}$ diam (without ornamentation), and thick-walled with stellate ornamentation (Fig. 19). The suspensors were equally shaped. The occurrence of progametangial stages could not be evaluated because with their light pigmentation they were only recognisable if they were formed in addition to zygospores. Repeated mating of reference strains (Table S3) resulted in zygospores with similar diameter and ornamentation. With two exceptions (CBS $172.27 \times$ CBS 116.08, CBS $192.68 \times$ CBS $116.08)$, an equal number of zygospores was formed in subsequent crosses of the same pairs of strains.

Zygospores were observed in 17 intra PS crosses (33\%) and 30 inter PS crosses (20 \%) (Fig. 20, Table S1). Using current taxonomic concepts, mating was successful in 30 intraformae crosses (39\%) and 17 (25\%) interformae crosses. Inter PS crosses were observed for all pairs of PS that were assigned to the same forma: PS 1 and 2 (f. janssenii), PS 7 and 8 (f. griseocyanus), and PS 14 and 15 (f. circinelloides). However, inter PS crosses also occurred between PS of different formae: PS 14 and 15 (f. circinelloides) were able to mate with all other formae, while no zygospores were formed in crosses between
PS that were not assigned to formae (PS 4, PS 6, PS 11). In our experimental setting, PS 10 (f. lusitanicus) was only able to mate with PS 14 (f. circinelloides) but not with PS 1 and 2 (f. janssenii) and PS 7 and 8 (f. griseocyanus); nevertheless PS 8 (f. griseocyanus) and PS 1 and 2 were able to mate with each other. No zygospore formation was observed in pairings between strains of PS 4 and 9 . The highest number of inter PS matings (6) was observed with the multipotent strain CBS 116.08 in PS 8. In crosses of f. circinelloides CBS 172.27 (PS 15) $\times \mathrm{f}$. janssenii CBS 232.29 (PS 1) only early states of zygospore formation (gametangia) were observed. For 13 strains the mating type could be assigned (Table S1). An overview of all confronted strains is given in Table S2.

Fig. 21a shows abundance, size and height of ornamentation of zygospores formed in intra and inter PS crosses; some data are missing because some low-numbered zygospores were damaged during slide preparation. Zygospore size and height of ornamentation are correlated. No obvious difference between intra and inter PS crosses was revealed. For example, intra PS zygospores of PS 14 (light pink circles) and PS 15 (vinaceous circles) were similar in number, size and ornamentation to 

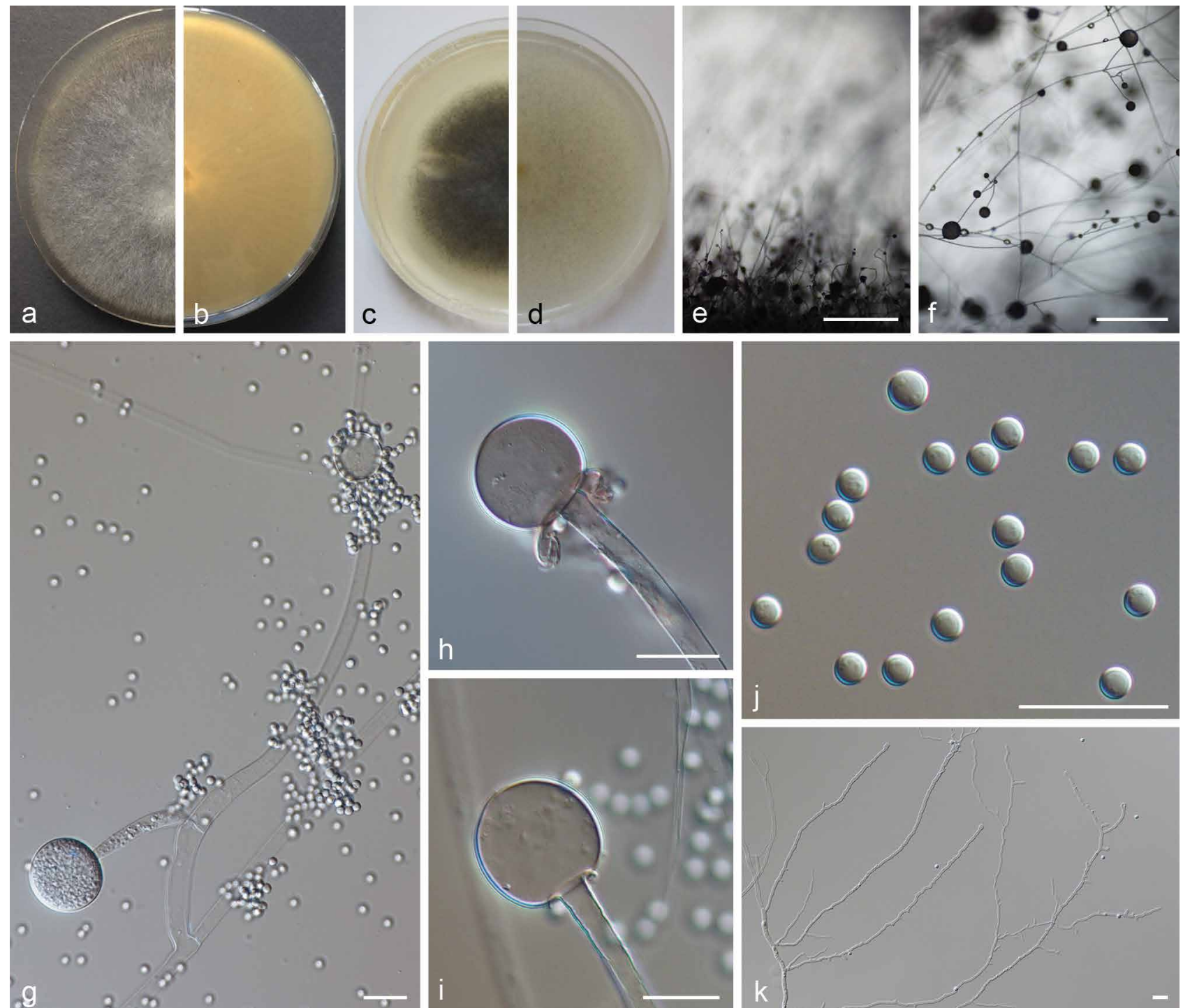

Fig. 14 PS 9 (M. amethystinus sp. nov.) CBS 846.73. a. Front and b. reverse of a 7-d-old culture on MEA at RT; c. front on PDA; d. front on SMA; e. lateral view; $f$. top view; $g$. side branch of a sporangiophore; $h-i$. columella with collar; j. sporangiospores; $k$. rhizoids. - Scale bars: $e-f=500 \mu m ; g-k=20 \mu m$.

zygospores formed between PS 14 and PS 15 (red circles). However, using of current taxonomic concepts (Fig. 21b), interformae crosses (white, grey and black circles) generally resulted in less zygospores compared to intraformae crosses (coloured circles). Often the contact zone between mating partners of interformae crosses showed a yellow pigment. In f. circinelloides (Fig. 21c) interformae crosses (black and dark grey circles) differed from intraformae crosses (circles in shades of red) not only by a lower number of zygospores but also by smaller zygospores with lower ornamentation. The situation might be similar in f. griseocyanus (Fig. 21b, intraformae zygospores blue circles) and f. lusitanicus (Fig. 21b, intraformae zygospores yellow circles) but the number of successful crosses was too low to see a clear difference. In $\mathrm{f}$. janssenii (PS 1 and PS 2) (Fig. 21d) there is no difference in size and ornamentation of intra- (circles in shades of green) and interformae (white and dark grey circles) zygospores which might be due to the fact that also the intraformae zygospores of $f$. janssenii are already small with a low ornamentation.

\section{Zygospore germination}

Germination of zygospores obtained in our mating experiments was tested to prove their ability to develop progeny. Zygospores produced within f. circinelloides, i.e., CBS 394.68 (PS 15) $\times$ CBS 172.27 (PS 15) and CBS 394.68 (PS 15) $\times$ CBS 192.68
(PS 14), were used. In our first experimental setting using zygospores harvested after $4 \mathrm{wk}$ that were plated on MEA plates, no germ sporangia were observed. Zygospores of CBS 394.68 $\times$ CBS 172.27 (5 times) and CBS $192.68 \times$ CBS 394.68 (once) germinated with vegetative hyphae (Fig. 22). Hence, we could show viability and germination capacity of these zygospores, but not the occurrence of meiosis and progeny. With the second approach using zygospores harvested after $10 \mathrm{~d}$ and plated on agarose plates with $\mathrm{pH} 4$ the zygospores of CBS $394.68 \times$ CBS 172.27 (two times) germinated with germ sporangia after six d (Fig. 23).

\section{Synopsis of the results and taxonomy}

Phylogenetic analyses of five markers detected 16 PS in the MCC. The formae consisted of one PS (f. lusitanicus) or two PS (f. circinelloides, f. griseocyanus, f. janssenii). For f. circinelloides we found that intraformae zygospores are on average larger and formed in higher number supporting the species status of the forma. For $f$. lusitanicus and f. griseocyanus we lacked enough positive crosses for a final conclusion but numbers and measurements of the observed zygospores are in line with the results for $\mathrm{f}$. circinelloides. In addition, we did not detect any phenotypical differences between the two PS within f. circinelloides (PS 14 and 15) and f. griseocyanus (PS 7 and 8). Because of the lacking recombination among the formae, their 

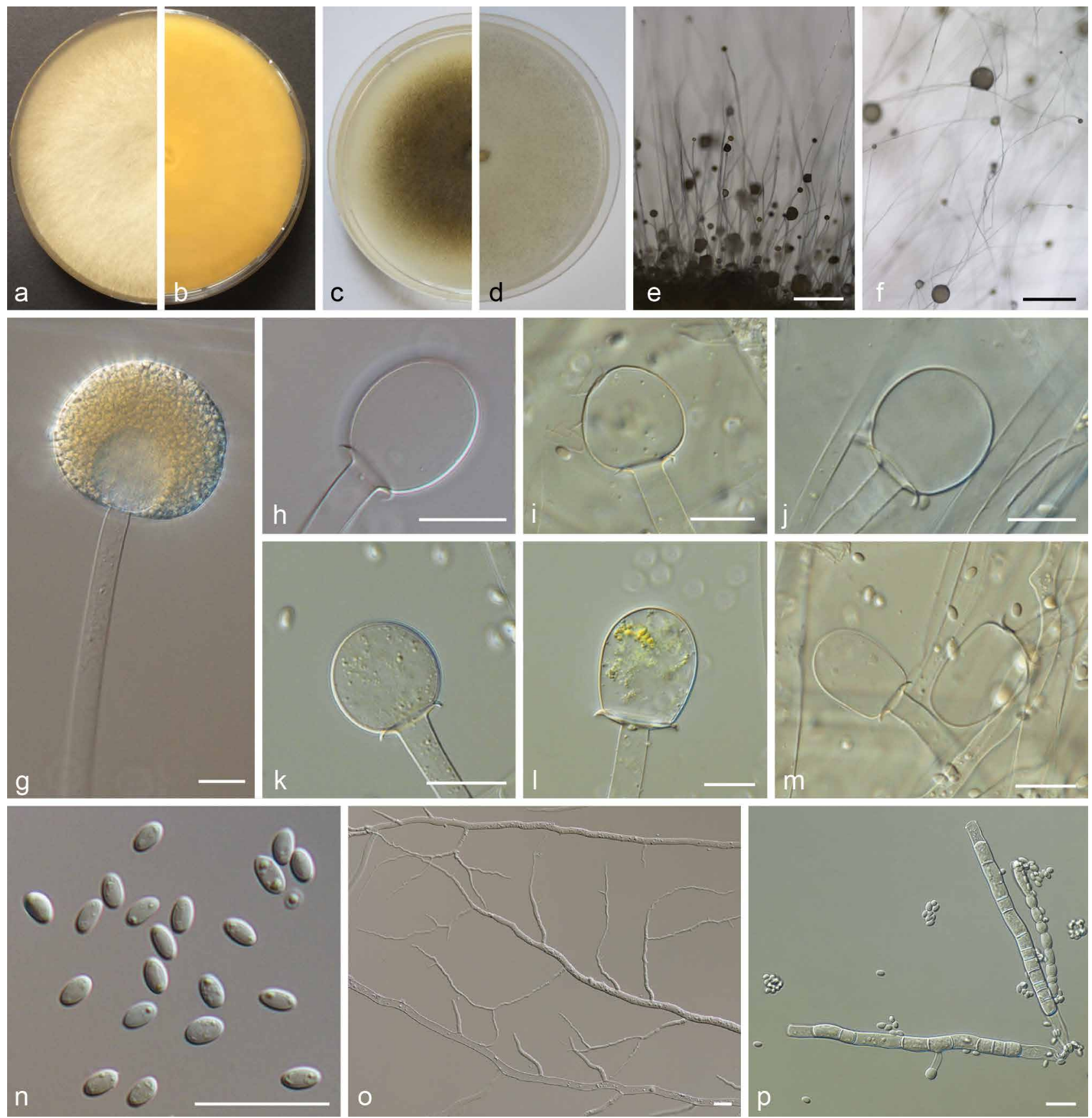

Fig. 15 PS 11 (M. variicolumellatus sp. nov.) CBS 236.35 and JMRC:SF:012536. a. Front and b. reverse of a 7-d-old culture on MEA at RT; c. front on PDA; d. front on SMA; e. lateral view; f. top view; g. sporangium with translucent columella; $h-m$. columellae; $n$. sporangiospores; o. rhizoid; $p$. chlamydospores. - Scale bars: $e-f=500 \mu m ; g-p=20 \mu m$.

phenotypic distinctness and the differences in size, number and ornamentation of the intraformae zygospores described above we consider these formae as discrete species.

In f. janssenii, both detected PS (PS 1 and 2) possessed a characteristic morphology but the intra PS zygospores did not differ from inter PS zygospores in this forma which might be due to the fact that also intraformae zygospores of $\mathrm{f}$. janssenii are already small. Based on the number of case reports (see discussion for details) PS 2 (M. velutinosus) is by far more clinically important than PS 1 (M. janssenii). Due to their specific morphology and apparent differences in their clinical relevance we also accept them as discrete species. Hence, each former forma of $M$. circinelloides obtains the status of one or of two species. The remaining nine PS detected showed all characteristic phenotypic features that were not intermediate between formae. In addition, there were no zygospores formed in inter PS crosses of these PS. Consequently, these PS are also considered as discrete species. Five of these species were newly described below.

We propose to accept the following species:

Ellisomyces anomalus (Hesselt. \& P. Anderson) Benny \& R.K. Benj., Aliso 8: 330. 1975 - MycoBank MB313478; Fig. $11,12 b$

Basionym. Thamnidium anomalum Hesselt. \& P. Anderson, Amer. J. Bot. 43: 699.1956.

Typus. USA, California, dung of lizard, unknown date, R.K. Benjamin (holotype NRRL 2465, culture ex-type NRRL $2465=$ CBS $243.57=$ ATCC $32879=$ IFO $6151=$ IMI $211277=$ RSA 88).

Descriptions \& Illustrations - Hesseltine \& Anderson (1956), Benny \& Benjamin (1975).

Habitat — Isolated from dung of lizard and mouse. 

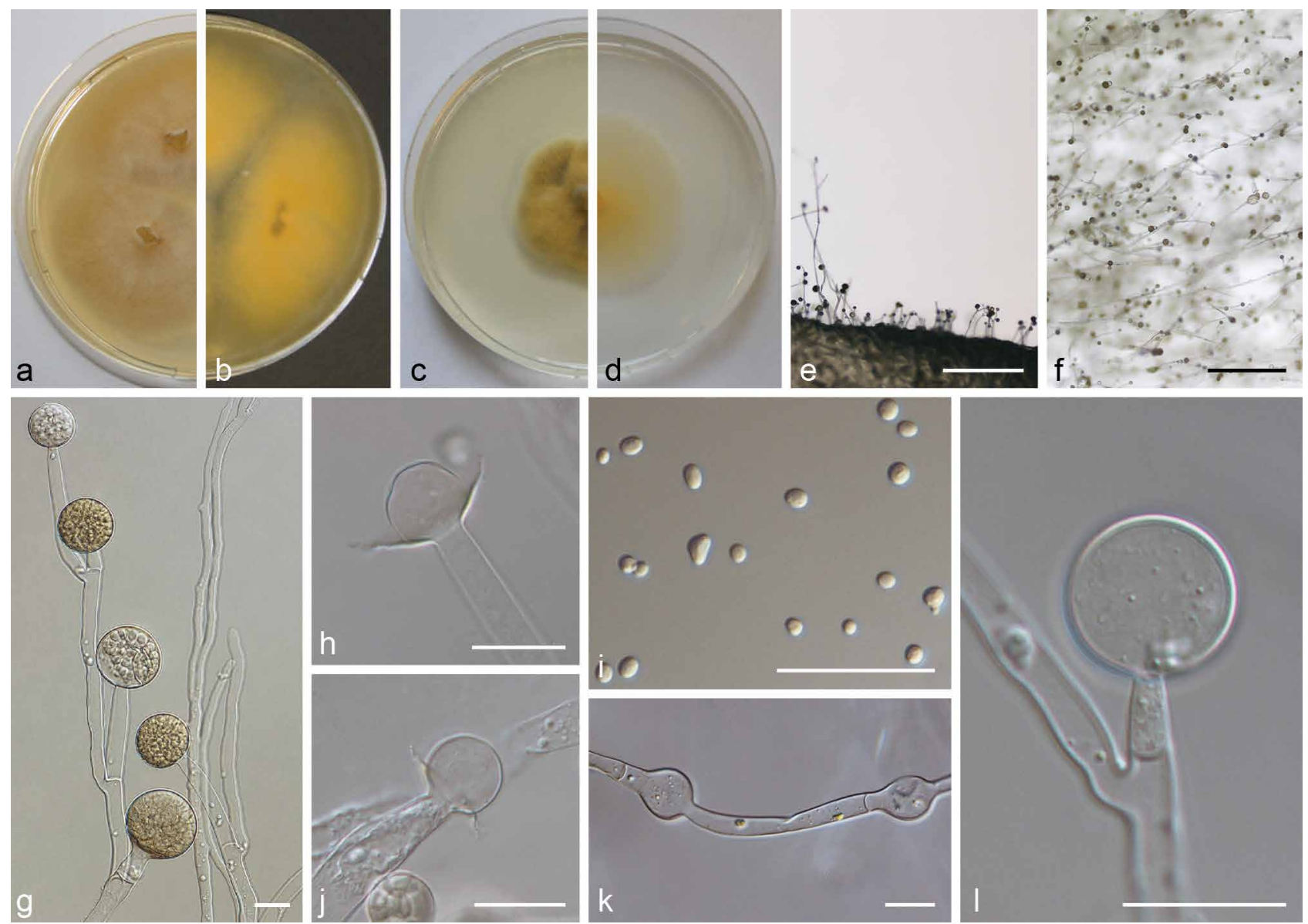

Fig. 16 PS 12 (M. ramosissiumus) CBS 135.65. a. Front and b. reverse of a 7-d-old culture on MEA at RT; c. front on PDA; d. front on SMA; e. lateral view; f. top view; g. sporangiophore with sporangia; $h$, j. columellae with collar; i. sporangiospores; $k$. hyphal bulges; I. sterile sporangium. - Scale bars: $e-f=$ $500 \mu \mathrm{m} ; \mathrm{g}-\mathrm{I}=20 \mu \mathrm{m}$.
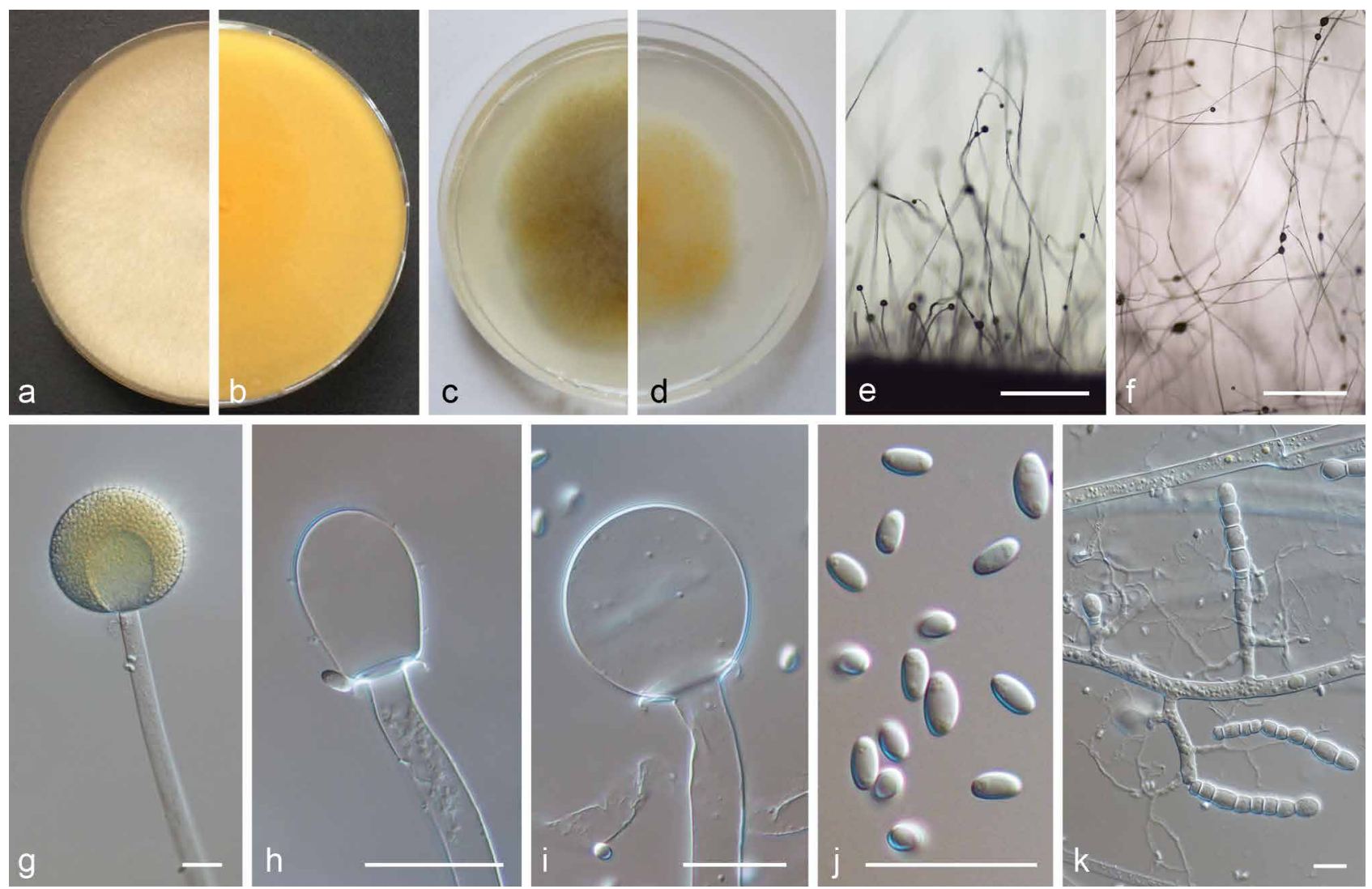

Fig. 17 PS 13 (M. pseudocircinelloides sp. nov.) CBS 541.78. a. Front and b. reverse of a 7-d-old culture on MEA at RT; c. front on PDA; d. front on SMA; e. lateral view; f. top view; g. sporangium with translucent columella; $h-i$. columella; $j$. sporangiospores; $k$. rhizoids and chlamydospores. $-S c a l e$ bars: $e-f$ $=500 \mu \mathrm{m} ; \mathrm{g}-\mathrm{k}=20 \mu \mathrm{m}$. 

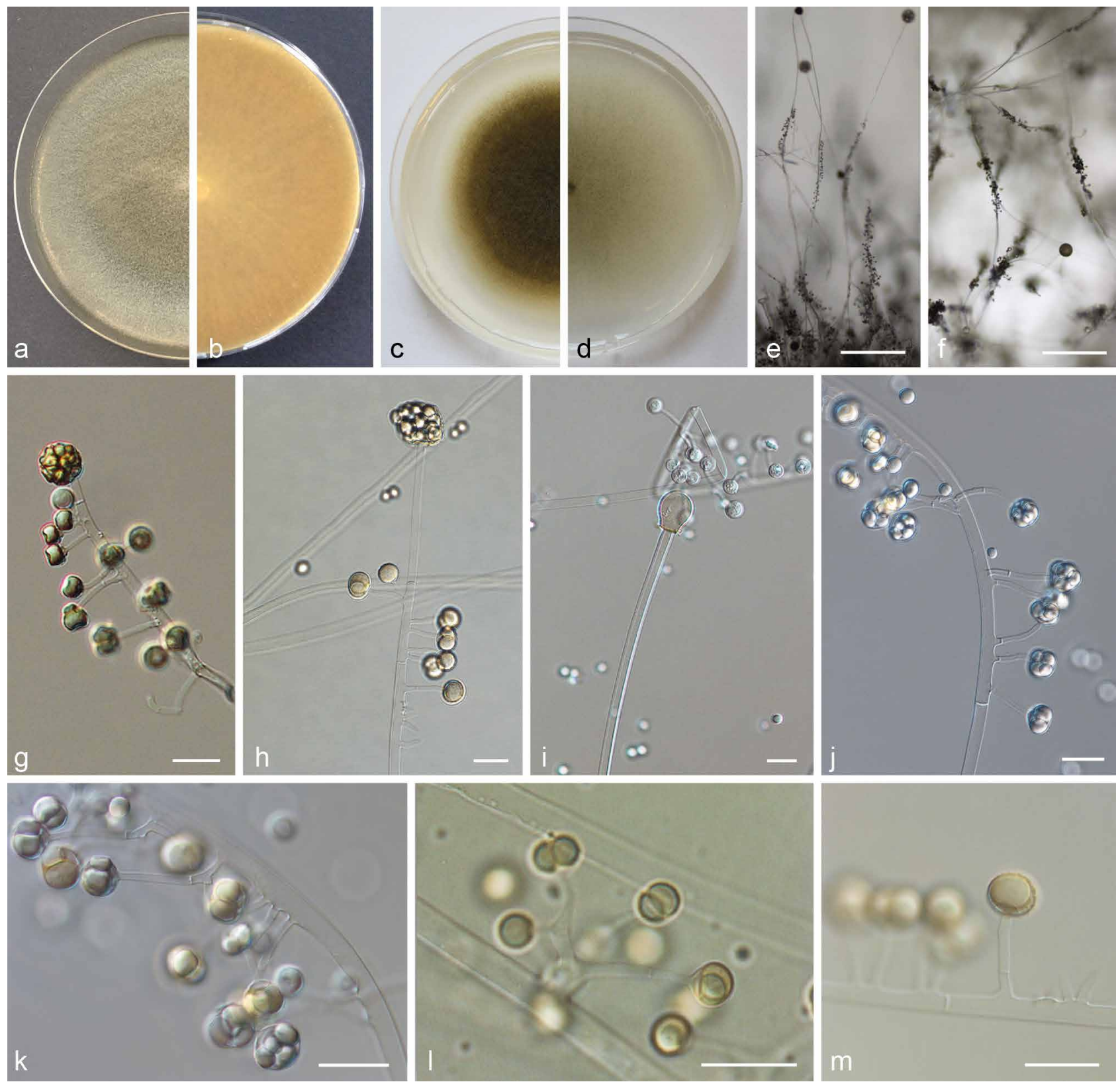

Fig. 18 PS 16 (M. ctenidius) CBS 293.66. a. Front and b. reverse of a 7-d-old culture on MEA at RT; c. front on PDA; d. front on SMA; e. lateral view; f. top view; $\mathrm{g}-\mathrm{h}$. sporangiophore with terminal sporangium; i. columella; $\mathrm{j}-\mathrm{k}$. sporangiola; I. sporangiola of CBS 433.87 with columella; $\mathrm{m}$. sporangiola. - Scale bars: $e-f=500 \mu m ; g-m=20 \mu m$

Distribution - California (USA).

Additional material examined. USA, California, dung of mouse, unknown date, R.K. Benjamin (CBS $697.76=$ ATCC $32880=$ NRRL $6244=$ RSA 669).

Mucor amethystinus L. Wagner \& G. Walther, sp. nov. - MycoBank MB828290; Fig. 14

Etymology. Refers to the colour of the growing zone of the mycelia.

Typus. ArmeniA, Goris, from soil, 1968, A.A. Milko (holotype CBS H-23929, culture ex-type CBS 526.68 = IMI 133975 = VKM F-1263)

Colonies on MEA cottony, flat, up to $5(-6) \mathrm{mm}$ high, sometimes reaching the lid of the Petri dish in the centre, reaching $5 \mathrm{~cm}$ diam after $2 \mathrm{~d}$, dark grey (2.5Y 4/0) with tinge of violet especially in the growing zone, reverse uncoloured. Sporangiophores of the small and tall type present; tall sporangiophores erect, up to $19 \mu \mathrm{m}$ diam, branched with small, strongly sympodially branched side branches. Sporangia non-apophysate, globose, black, large, up to $160 \mu \mathrm{m}$ diam, with deliquescent walls. Columella globose to slightly elongated or applanate, 33-54 $\times$ 39-65 $\mu \mathrm{m}$, hyaline to brown, with distinct brown collar. Sporan- giospores regularly shaped, globose to subglobose, 4-5(-6) $\mu \mathrm{m}$, slightly rough. Chlamydospores occurring in vegetative hyphae, intercalary, singly or in chains of up to 4, thick-walled, predominantly ellipsoidal or rarely subglobose, 10.4-39.5 $\times$ 6.9-17.3 $\mu \mathrm{m}$. Rhizoids present, pronounced when growing on glass slides. Zygospores not observed. Maximum growth temperature $36^{\circ} \mathrm{C}$ (without sporulation). Colonies on PDA similar to colonies on MEA but with uncoloured sectors and slightly larger sporangia reaching up to $175(-215) \mu \mathrm{m}$ diam, columellae globose to slightly elongated or obovoid. Colonies on SMA also similar to those on MEA but with smaller sporangia reaching up to $100(-115) \mu \mathrm{m}$ diam, columellae globose to slightly elongated or obovoid. Yeast state present in liquid YPG medium.

Habitat — Isolated from soil.

Distribution - Armenia, Chile.

Additional material examined. CHILE, from soil, 1973, J. Grinbergs (CBS 846.73).

Notes - Mucor amethystinus is one of the MCC species that forms dark and flat mycelia. It resembles $M$. janssenii but in contrast to the latter species the mycelia of $M$. amethystinus 


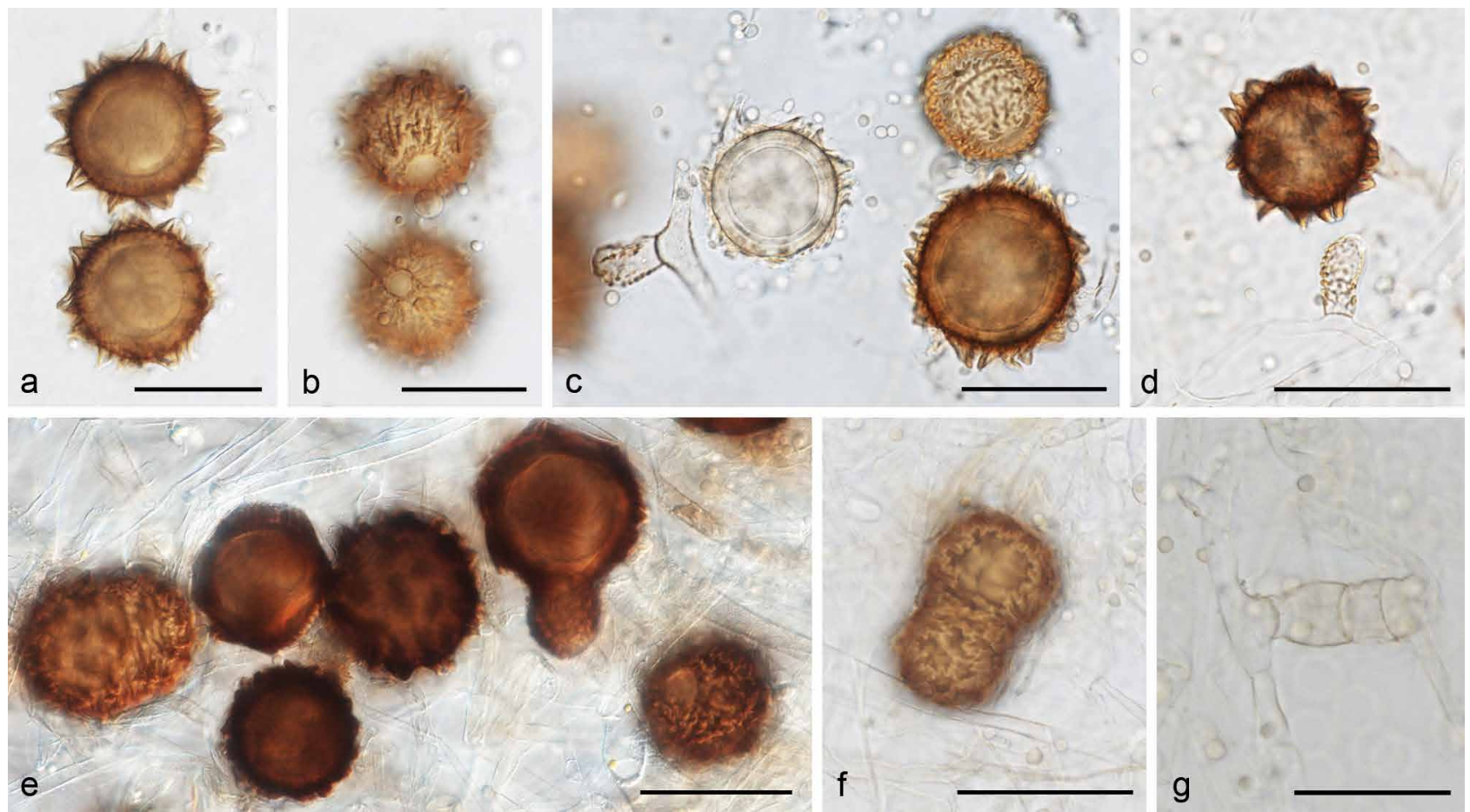

Fig. 19 Zygospores of the MCC. a-d. Well-developed zygospores (a-c. zygospores of intraformae cross within f. circinelloides CBS $394.68 \times$ CBS 172.27 : C. different states of zygospore development (gametangium, young zygospore, maturing zygospore, matured zygospore); d. zygospores of intraformae cross within f. circinelloides CBS $394.68 \times$ CBS 192.68); e-f. not well-developed zygospores (e. interformae cross between f. griseocyanus CBS 116.08 and f. circinelloides CNM-CM3112; f. zygospores of interformae cross between f. griseocyanus CBS 116.08 and f. janssenii CBS 205.68); g. gametangia of interformae cross between f. griseocyanus CBS 116.08 and f. janssenii CBS 205.68. - Scale bars $=50 \mu \mathrm{m}$.

have a violet tinge, its sporangia are much larger, and it develops predominantly globose columellae while the columellae of M. janssenii are distinctly elongated.

\section{Mucor atramentarius L. Wagner \& G. Walther, sp. nov. - Myco-} Bank MB828291; Fig. 13

Etymology. Refers to the dark colour of the sporangia.

Typus. UNKNOWN COUNTRY, unknown source and date, unknown collector (holotype CBS H-23927, culture ex-type CBS 202.28).

Colonies on MEA cottony, flat, up to $3 \mathrm{~mm}$ in height at the point of inoculation, slow growing, reaching $3.5 \mathrm{~cm}$ diam after $2 \mathrm{~d}$, young cream-coloured (10YR 8/3), later becoming grey (10YR $7 / 2$ ) in well-defined sectors while other sectors staying creamcoloured because of lacking sporulation (Fig. 13a); reverse uncoloured. Sporangiophores of the small and tall type present; tall sporangiophores erected, but bending towards the light, up to $16 \mu \mathrm{m}$ diam, young rarely branched, in old cultures frequently monopodially branched; small sporangiophores sympodially branched. Sporangia non-apophysate, globose, up to $70 \mu \mathrm{m}$ diam, dark brown to black, with deliquescent or disruptive walls. Columellae globose, slightly ellipsoidal or applanate, brown, $25-40 \times 25-38 \mu \mathrm{m}$, with distinct brown collar. Sporangiospores globose to subglobose, regularly shaped, homogenously in size, 3.5-5(-6) $\mu \mathrm{m}$ diam, hyaline, smooth-walled. Zygospores not observed. Rhizoids present. Chlamydospores absent. Maximum growth temperature $34^{\circ} \mathrm{C}$ (without sporulation). Colonies on PDA higher, reaching the lid after 2 wk, grey; sporangia (up to $90 \mu \mathrm{m}$ ) and columellae (up to $50 \times 45 \mu \mathrm{m}$ ) slightly larger than on MEA. Colonies on SMA only grey at the point of inoculation (sporangia only formed here), white-yellow in the remaining areas, with reduced growth compared to MEA and PDA, sporangia (up to $90 \mu \mathrm{m}$ ) and columellae (up to $50 \times 45 \mu \mathrm{m}$ ) slightly larger than on MEA. Yeast state absent in liquid YPG medium.
Habitat - Unknown

Distribution - Unknown.

Notes - Mucor atramentarius is a species with rather flat, light coloured mycelia and restricted growth. Striking are the differently coloured sectors of the colonies. In contrast to M. ramosissimus it forms only globose to subglobose sporangiospores.

\begin{tabular}{|c|c|c|c|c|c|c|c|c|c|c|c|c|c|c|c|}
\hline+ & 穴 & $\begin{array}{l}N \\
\mathcal{N} \\
\Omega\end{array}$ & $\begin{array}{l}n \\
\tilde{n}\end{array}$ & $\begin{array}{l}\nabla \\
\mathcal{S} \\
\square\end{array}$ & $\begin{array}{l}0 \\
\mathcal{L} \\
0\end{array}$ & $\begin{array}{l}0 \\
\widetilde{Q} \\
\Omega\end{array}$ & $\begin{array}{l}\lambda \\
\mathcal{D} \\
\Omega\end{array}$ & $\begin{array}{l}\infty \\
\infty \\
\square\end{array}$ & $\begin{array}{l}0 \\
\infty \\
\square\end{array}$ & $\begin{array}{l}\stackrel{0}{0} \\
\tilde{\Omega}\end{array}$ & $\begin{array}{l}\check{\sigma} \\
\tilde{\Sigma}\end{array}$ & $\begin{array}{l}\stackrel{N}{\infty} \\
\tilde{L}\end{array}$ & $\begin{array}{l}m \\
\stackrel{n}{n} \\
n\end{array}$ & $\begin{array}{l}\stackrel{\nabla}{~} \\
\stackrel{\sim}{0}\end{array}$ & $\begin{array}{l}\stackrel{n}{0} \\
\infty\end{array}$ \\
\hline PS 1 & & & & & & & & & & & & & & & \\
\hline PS 2 & & & & & & & & & & & & & & & \\
\hline PS 3 & & & & & & & & & & & & & & & \\
\hline PS 4 & & & & & & & & & & & & & & & \\
\hline PS 5 & & & & & & & & & & & & & & & \\
\hline PS 6 & & & & & & & & & & & & & & & \\
\hline PS 7 & & & & & & & & & & & & & & & \\
\hline PS 8 & & & & & & & & & & & & & & & \\
\hline PS 9 & & & & & & & & & & & & & & & \\
\hline PS 10 & & & & & & & & & & & & & & & \\
\hline PS 11 & & & & & & & & & & & & & & & \\
\hline PS 12 & & & & & & & & & & & & & & & \\
\hline PS 13 & & & & & & & & & & & & & & & \\
\hline PS 14 & & & & & & & & & & & & & & & \\
\hline PS 15 & & & & & & & & & & & & & & & \\
\hline
\end{tabular}

Fig. 20 Overview of performed crosses. PS arranged according to their mating types (+/-): orange - formation of inter PS zygospores, blue - formation of intra PS zygospores, grey - cross without formation of zygospores, white - no cross performed. 


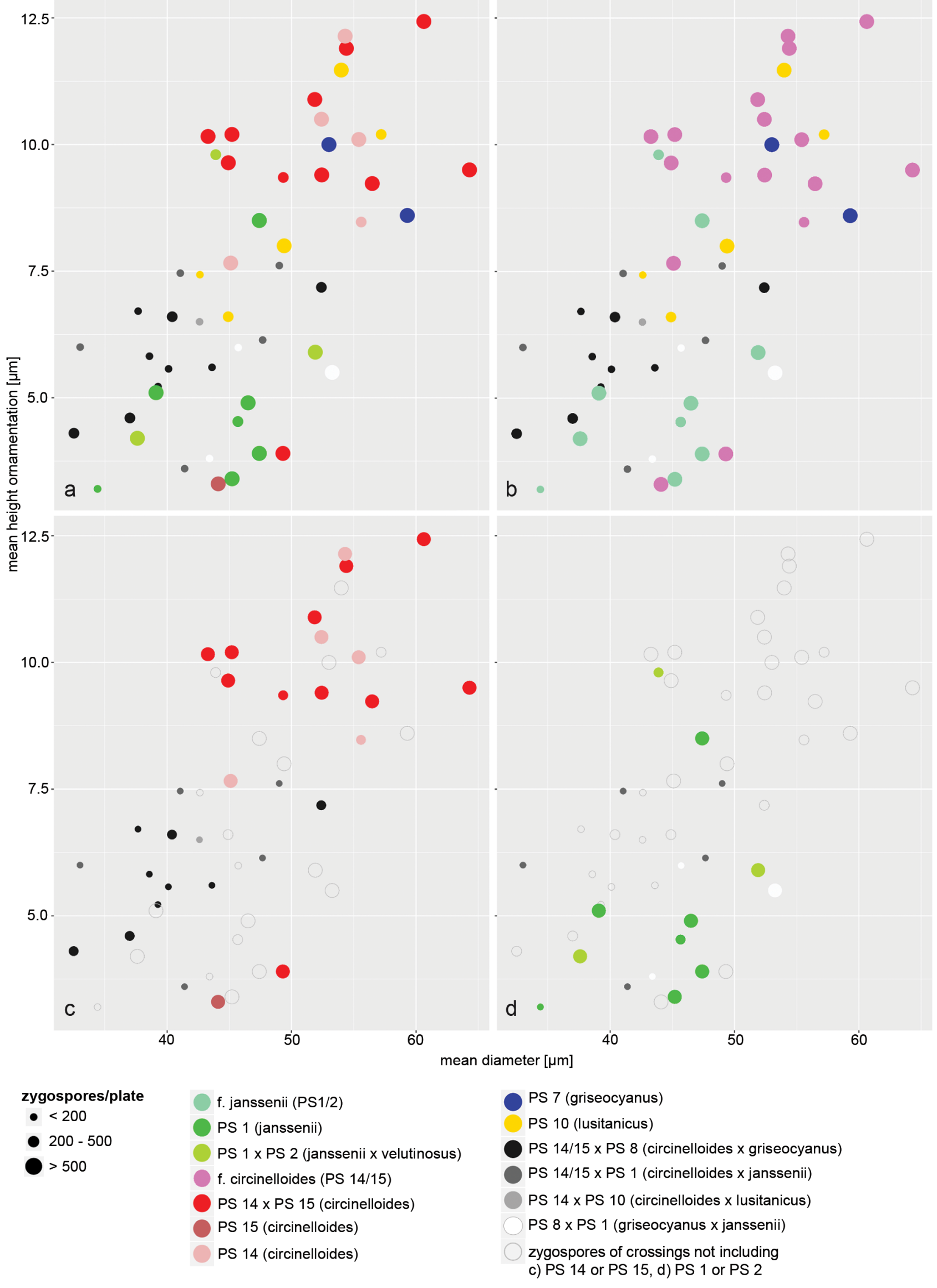

Fig. 21 Size, height of ornamentation and abundance of zygospores formed in the MCC. a. Intra- and inter PS crosses; b. intra- and interformae crosses; c. intra- and inter PS crosses (PS $14 \times$ PS 15) of f. circinelloides; d. intra- and inter PS crosses (PS 1, M. janssenii $\times$ PS 2, M. velutinosus) of f. janssenii. 

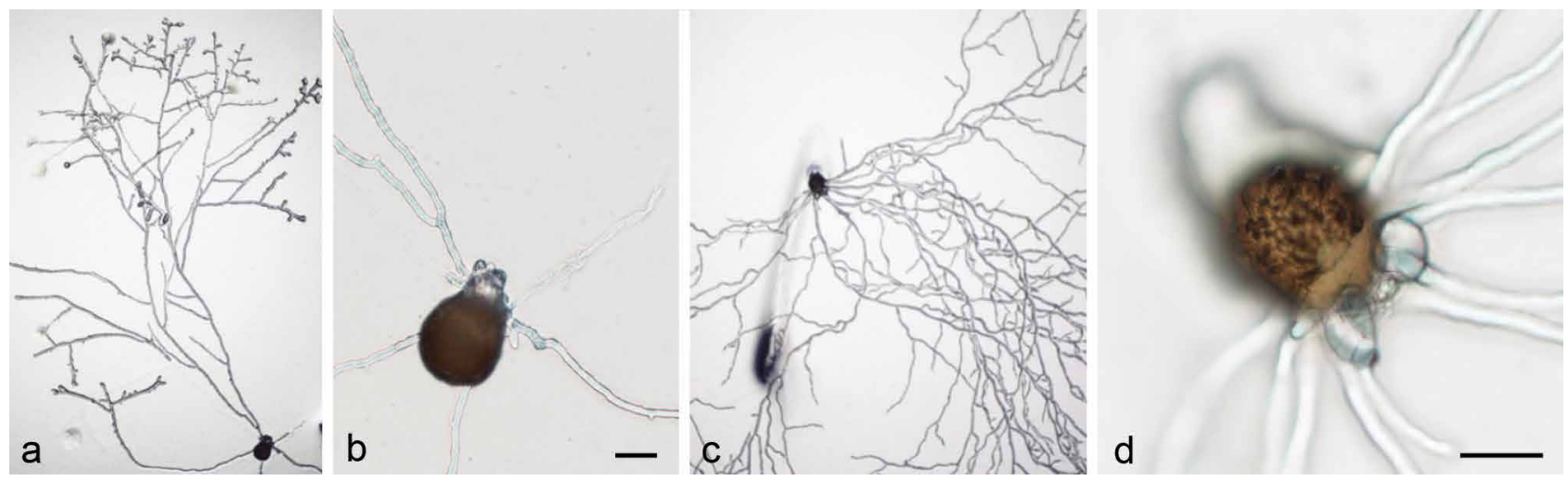

Fig. 22 a-d. Mycelial germination of zygospores of crosses within f. circinelloides (PS 15, CBS 394.68 $\times$ CBS 172.27). - Scale bars $=20 \mu \mathrm{m}$.

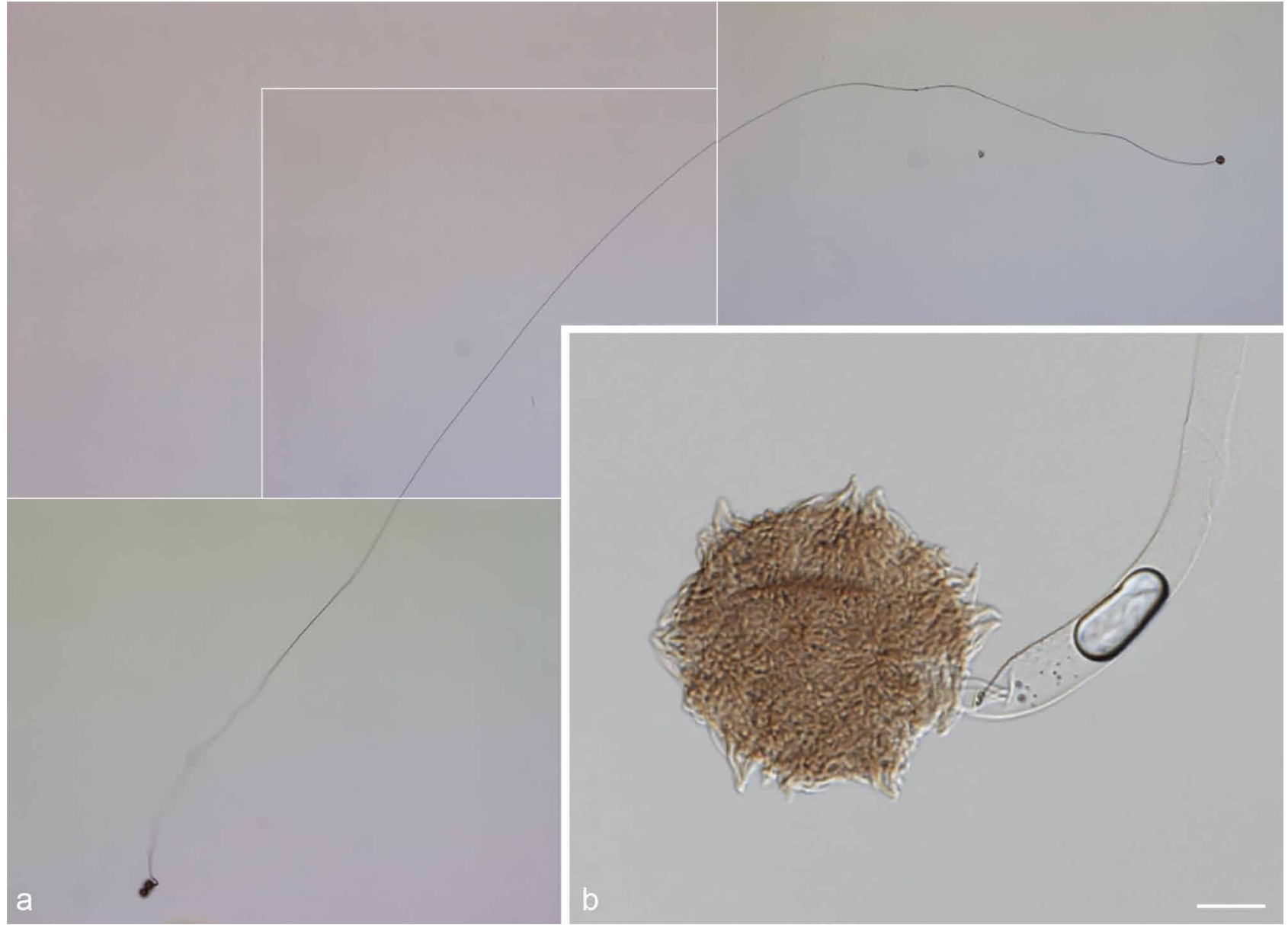

Fig. 23 Sporangial germination of a zygospore of crosses within f. circinelloides (PS 15, CBS $394.68 \times$ CBS 172.27). a. Germ sporangiophore, total height about $1 \mathrm{~cm}$ (photographs assembled); b. zygospore with basal part of germ sporangiophore. - Scale bar $=20 \mu \mathrm{m}$.

Mucor bainieri B.S. Mehrotra \& Baijal, Aliso 5: 237. 1963 MycoBank MB334522; Fig. 8

Synonyms. Circinomucor bainieri (B.S. Mehrotra \& Baijal) Arx, Sydowia 35: 18.1982.

Mucor tenuis Bainier, Ann. Sci. Nat., Bot., sér. 6, 15: 353. 1883, nom. illeg. (Art. 53.1), non Mucor tenuis Link (1824).

Mucor racemosus var. tenuis Burgeff, Bot. Abh. 4: 41. 1924.

Typus. INDIA, Uttar Pradesh, near Ranikhet, forest soil, unknown date, R.K. Benjamin, isolated by B.S. Mehrotra \& U. Baijal (isotype CBS H-7368, culture ex-isotype CBS 293.63 = ATCC $15088=$ CCRC $32080=$ IMI 101214 $=$ NRRL A-11496 $=$ RSA $1210=$ VKM F-1215).

Descriptions \& Illustrations - Benjamin \& Mehrotra (1963), Schipper (1976b).
Habitat - Isolated from forest soil.

Distribution - India.

Mucor circinelloides Tiegh., Ann. Sci. Nat., Bot., sér. 6, 1: 94. 1875 - MycoBank MB198947; Fig. 6

Synonyms. Mucor circinelloides f. circinelloides Tiegh., Ann. Sci. Nat., Bot., sér. 6, 1: 94. 1875.

Mucor circinelloides var. circinelloides Tiegh., Ann. Sci. Nat., Bot., sér. 6, 1: 94.1875.

Calyptromyces circinelloides (Tiegh.) Sumst., Mycologia 2: 148. 1910. Circinomucor circinelloides (Tiegh.) Arx, Sydowia 35: 18. 1982.

Mucor griseoroseus Linnem., Flora, Regensburg 130: 189. 1936.

Rhizomucor variabilis var. regularior R.Y. Zheng \& G.Q. Chen, Mycosystema 6: 2. 1993. 
Rhizomucor regularior (R.Y. Zheng \& G.Q. Chen) R.Y. Zheng et al., Sydowia 61: 144. 2009.

Typus. NetherLANDS, Vlagtwedde, air, unknown date, G.A. de Vries (neotype CBS 195.68, culture ex-neotype CBS 195.68).

\section{Descriptions \& Illustrations - Van Tieghem (1875), Schipper} (1976b).

Habitat - Isolated from different types of soil (Schipper 1976b, Domsch et al. 1980, Connolly et al. 2010, Hermet et al. 2012, Walther et al. 2013, Mousavi et al. 2018), excrements of cattle, goat, sheep (De Souza et al. 2017), pig (Walther et al. 2013), and platypus (Connolly et al. 2010), plants including vegetables and fruit such as Artocarpus fruit, Capsicum annum, Triticum aestivum (Walther et al. 2013), mangoes, peaches, yams, hazelnuts, walnuts, maize, mung beans, soybeans and barley (Pitt \& Hocking 2009, Morin-Sardin et al. 2017), meat (Pitt \& Hocking 2009), processed food such as cheese (Hermet et al. 2012), and yogurt (Lee et al. 2014, Snyder et al. 2016), commercial honey, fermenting rice (component of 'Chinese yeast') (Walther et al. 2013), fermenting soybeans (starter cultures of sufu, Han et al. 2004), Homo sapiens (Álvarez et al. 2009, 2011, Walther et al. 2013).

Distribution - Africa, America, Asia, Australia, Europe.

Additional materials examined. Belgium, Homo sapiens (leg wound), 2007, unknown collector (CBS 124429); H. sapiens, unknown date, unknown collector (CNM-CM5169); Brussels, H. sapiens (wound), unknown date, unknown collector (IHEM 20006); Brussels, $H$. sapiens (burn wound), unknown date, unknown collector (IHEM 21158); Brussels, H. sapiens (burn wound), unknown date, unknown collector (IHEM 21426); Brussels, H. sapiens, unknown date, unknown collector (IHEM 22323). - CHINA, Hebei, H. sapiens (face), 1992, D.I. Wang (culture ex-type of Rhizomucor regularior CBS 384.95); Fujian, Chinese sufu starter, unknown date, unknown collector (CBS 111555 = AS 3.222). - FINLAND, soil, 1969, unknown collector (State Inst. for Techn. Res. Otaniemi) (CBS 479.70). - FRANCE, Lorraine, soil, unknown date, P. Lectard (CBS 606.78); Angers, unknown source and date, unknown collector (IHEM 21105). - GERMANY, soil, unknown date, G. Linnemann (culture ex-type of $M$. griseoroseus CBS $239.35=$ VKM F-1234); Marburg, air, unknown date, $H$. Zycha (CBS 247.35); thawing meat, 1965, R. Hadlok (CBS 394.68 = CCF 2012 = DSM 2183 = NRRL 3615); Gießen, on Capsicum annum, unknown date, R. Hadlok (CBS 480.70I); Berlin, $H$. sapiens (thigh necrosis), unknown date, B. Graf (CBS 123973); tracheal secretion ex joint from $H$. sapiens, Oct. 2015, unknown collector (JMRC:NRZ:0227); H. sapiens (deep wound smear), Aug. 2016, unknown collector (JMRC:NRZ:0474); H. sapiens (burn wound), Mar. 2017, unknown collector (JMRC:NRZ:0774); H. sapiens (burn wound), Apr. 2017, unknown collector (JMRC:NRZ:0797). - INDIA, Allahabad, soil, unknown date, B.S. Mehrotra (CBS 480.70F = NRRLA-12625). - ITALY, Milano, H. sapiens (skin), unknown date, unknown collector (IHEM 16415). - JAPAN, unknown source and date, unknown collector (CBS 108.16 = VKM F-1315). - NETHERLANDS, dung of pig, unknown date, unknown collector (CBS 192.68 = NRRL 3614). - Portugal, unknown source and date, T. Amaral (CBS 846.72A). - Spain, Reus, commercial honey, 2007, A.M. Stchigel (CBS 121702 = FMR 9399); $H$. sapiens (wound exudate), unknown date, unknown collector (CNMCM2922); peritoneal dialysis fluid ex joint from $H$. sapiens, unknown date, unknown collector (CNM-CM3112); $H$. sapiens (reservoir), unknown date, unknown collector (CNM-CM3510); catheter ex joint from $H$. sapiens, unknown date, unknown collector (CNM-CM3785); $H$. sapiens (skin), unknown date, unknown collector (CNM-CM4299). - TURKEY, Ankara, on Triticum aestivum, unknown date, unknown collector (CBS 196.68). - UKRAINE, Lwow, unknown source and date, Swederski (CBS 295.34). - VIETNAM, Sa Dec Town, sufu, unknown date, unknown collector (CBS 111560); Chau Thanh district, sufu, unknown date, unknown collector (CBS 111561). - UNKNOWN COUNTRY, on Artocarpus fruit, unknown date, unknown collector (CBS 172.27 = IFO 5775); component of 'Chinese yeast', unknown date, unknown collector (CBS 416.77 = ATCC 24905 = CCM F-220 = CECT 2655 = DSM 1191); unknown source and date, unknown collector (CBS 464.78 = ETH 2605); unknown source and date, unknown collector (CBS 480.70G = ATCC 11010); unknown source and date, unknown collector (CNM-CM2437); unknown source and date, unknown collector (CNM-CM4249); unknown source and date, unknown collector (CNM-CM5071).
Mucor ctenidius (Durrell \& M. Fleming) G. Walther \& de Hoog, Persoonia 30: 41. 2013 - MycoBank MB800455; Fig. $12 \mathrm{c}-\mathrm{d}, 18$

Basionym. Thamnidium ctenidium Durrell \& M. Fleming, Mycologia 58: 797. 1966.

Synonym. Backusella ctenidia (Durrell \& M. Fleming) Pidopl. \& Milko ex Benny \& R.K. Benj., Aliso 8: 325. 1975.

Typus. USA, desert of Death Valley, desert soil, unknown date, L.W. Durrell (isotype CBS H-6778, culture ex-isotype CBS 293.66 = ATCC $16377=$ IMI 211279 = NRRL 6239)

Description \& Illustration — Durrell \& Fleming (1966).

Habitat - Isolated from dead plant material and soil. Distribution - Kenya, USA.

Additional materials examined. KENYA, dead plant material, unknown date, unknown collector (CBS 433.87). - USA, Nevada, dung of pack rat, unknown date, unknown collector (CBS 696.76 = ATCC $32873=I \mathrm{MI} 208233=\mathrm{NRRL}$ $6238=$ RSA 1166)

\section{Mucor griseocyanus Hagem, Skr. Vidensk.-Selsk. Christiania,} Math.-Naturvidensk. KI. I (no. 7): 28. 1908 - MycoBank MB373691; Fig. 7

Synonyms. Mucor griseocyanus f. griseocyanus Hagem, Skr. Vidensk.Selsk. Christiania, Math.-Naturvidensk. KI. I (no. 7): 28. 1908.

Mucor circinelloides f. griseocyanus (Hagem) Schipper, Stud. Mycol. 12: 12. 1976.

Circinomucor griseocyanus (Hagem) Arx, Sydowia 35: 18. 1982.

Typus. Norway, near Oslo, humous soil, unknown date, O. Hagem, f. 9 in Hagem, Mathematisk-Naturvidenskabelig Klasse, no. 7: 29. 1908, lectotype designated here, MBT386681; soil, unknown date, O. Hagem (epitype CBS $\mathrm{H}-23926$, designated here, MBT386682, culture ex-epitype CBS $116.08=$ ATCC $22363=$ NRRL A-7420)

\section{Description \& Illustration - Schipper (1976b).}

Habitat — Isolated from forest soil, leaf litter, Zea mays, milk powder, canned strawberries (Schipper 1976b), excrements of cattle, goat, and sheep (De Souza et al. 2017).

Distribution - Brazil, Germany, India, Norway, South Africa, The Netherlands.

Additional materials examined. Germany, H. sapiens (nail), 2012, unknown collector (JMRC:SF:011463). - NETHERLANDS, unknown source and date, A.F. Blakeslee (CBS 223.56 = ATCC 1207a = IMI 116946); canned strawberries, unknown date, unknown collector (CBS 366.70). - SOUTH AfricA, Pretoria, on Zea mays, 1965, D.B. Scott (CBS 698.68). - UNKNOWN COUNTRY, unknown source and date, A.F. Blakeslee (CBS 198.28 = NRRL 3621).

Mucor janssenii Lendn. (as 'janseni'), Bull. Herb. Boissier, sér. 2, 7: 251. 1907 - MycoBank MB271799; Fig. 4

Synonyms. Mucor griseocyanus Hagem f. janssenii (Lendn.) Schipper, Antonie van Leeuwenhoek 36: 486. 1970.

Mucor circinelloides f. janssenii (Lendn.) Schipper, Stud. Mycol. 12: 13. 1976.

Circinomucor janssenii (Lendn.) Arx, Sydowia 35: 18. 1982.

Mucor tenellus Y. Ling, Rev. Gén. Bot. 42: 736. 1930.

Circinella tenella (Y. Ling) Zycha, Krypt.-FI. Brandenburg (Leipzig) 6.6: 99. 1935

Mucor kurssanovii Milko \& Beliakova, Mikrobiologija 36: 118. 1967.

Typus. South AfricA, KwaZulu-Natal, forest soil, 1966, A. Eicker (neotype CBS 205.68, preserved in a metabolically inactive state by lyophilisation, batch nr. 605, culture ex-neotype CBS 205.68 = ATCC $23362=$ NRRL 3826).

Description \& Illustration - Schipper (1976b).

Habitat - Isolated from forest and grassland soil (Schipper 1976b), excrements of cattle, goat, sheep (De Souza et al. 2017) and Carausius morosus (Schipper et al. 1976b), Homo sapiens (Walther et al. 2013).

Distribution - Brazil, China, France, India, Malaysia, Russia, South Africa, South Korea, Vietnam. 
Additional materials examined. FRANCE, unknown source and date, Y. Ling (culture ex-type of $M$. tenellus CBS 232.29 = VKM F-1321). - RussIA, Kuril Islands, grassland soil, unknown date, unknown collector (culture ex-type of M. kurssanovii CBS 185.68 = ATCC $18357=$ IMI $129970=$ NRRL $3302=$ VKM F-1092). - South AfriCA, Durban, H. sapiens (mucormycosis), 1959, K.C. Watson (CBS 243.67 = IMI 077436). - UNKNOWN COUNTRY, unknown source and date, N.A. Naumov (CBS 227.29 = NRRL 2629); dung of Carausius morosus, unknown date, unknown collector (CBS 365.70).

Mucor lusitanicus Bruderl., Bull. Soc. Bot. Genève, sér. 2, 8: 276. 1916 - MycoBank MB149125; Fig. 10

Synonyms. Mucor racemosus var. Iusitanicus (Bruderl.) Naumov, Opred. Mukor., Edn 2: 46. 1935.

Mucor circinelloides f. lusitanicus (Bruderl.) Schipper, Stud. Mycol. 12: 9. 1976.

Mucor griseolilacinus Povah, Bull. Torrey Bot. Club 44: 301. 1917

Mucor jauchae Lendn., Bull. Soc. Bot. Genève, sér. 2, 10: 374. 1918.

Mucor zeicolus P.W. Graff, CBS List of Cultures: 61. 1936, nom. inval.

Typus. Portugal, maize meal, unknown date, J. Bruderlein (lectotype f. 1 in Bruderlein, Bull. Soc. Bot. Genève, sér. 2, 8: 276. 1916, designated here, MBT386679). - UNKNOWN COUNTRY, unknown source and date, unknown collector, isolated by $A$. Lendner (epitype CBS H-23925, designated here, MBT386680, culture ex-epitype CBS 108.17).

Description \& Illustration - Schipper (1976b).

Habitat - Isolated from forest soil, Zea mays, maize meal (Schipper 1976b, Walther et al. 2013), fermented soybeans (sufu starter, Schipper 1976b, meju, Hong et al. 2012), excrements of cattle and sheep (De Souza et al. 2017), Homo sapiens (Álvarez et al. 2011).

Distribution - Brazil, China, France, Mexico, Portugal, Russia, South Africa, USA.

Additional materials examined. CHINA, Jiangsu, sufu starter, unknown date (CBS 111228 = AS 3.2233 = LU 2027 = RM 35); Guangdong, sufu starter, unknown collector (CBS 111229 = AS $3.2545=$ LU $2029=$ RM 36). - MEXICO, unknown source and date, Searle (CBS 968.68). - Portugal, unknown source and date, T. Amaral (CBS $847.72=$ INII 19). - RussIA, forest soil, unknown date, A.A. Milko (CBS 969.68 = VKM F-1152). - South AfrICA, on Zea mays, unknown date, D.B. Scott (CBS 633.65). - USA, Illinois, on Zea mays grain, unknown date, Graff (CBS 253.35 = VKM F-1238). - UNKNown COUNTRY, unknown source and date, unknown collector (CBS $108.19=$ NRRL A-7421 = VKM F-1241); unknown source and date, unknown collector (CBS 242.33 = MUCL 15443 = VKM F-1233); unknown source and date, A.F. Blakeslee (CBS $276.49=$ ATCC 1216a = IHEM $3426=$ NRRL 3629); unknown source and date, A.F. Blakeslee (CBS $277.49=$ ATCC $1216 \mathrm{~b}=$ MUCL 15438 = NRRL 3631); unknown source and date, unknown collector (CBS 851.71 = NRRL 1443); unknown source and date, unknown collector (CBS 852.71 = NRRL 1442).

\section{Mucor pseudocircinelloides L. Wagner \& G. Walther, sp. nov.} — MycoBank MB828294; Fig. 17

Etymology. Refers to its morphological similarity to Mucor circinelloides.

Typus. South AfricA, on Zea mays, unknown date, D.B. Scott (holotype CBS H-23931, culture ex-type CBS 541.78).

Colonies on MEA cottony, high, reaching the lid of the Petri dish, young white (10YR 7/1 10Y8/3 3d MEA) turning to pale brown (10YR 7/3) after $14 \mathrm{~d}$; reverse yellow (2.5Y 8/6). Sporangiophores of the small and the tall type present; tall sporangiophores erect, sometimes sympodially branched (1-2 times), 10-17 $\mu \mathrm{m}$ diam; short sporangiophores repeatedly sympodially branched. Sporangia non-apophysate, globose, pale orange, up to $90 \mu \mathrm{m}$ diam, with mainly deliquescent walls. Columella globose to obovoid, the bulk of large columellae obovoid, 27-46 $\times 34-58 \mu \mathrm{m}$, hyaline or light brown, with distinct collar. Sporangiospores regularly shaped, varying in size, ellipsoidal, 5.5-9.5 × 3-4.7 $\mu \mathrm{m}$, with a length/width relation of 1.7-2.3, thin-walled, hyaline. Chlamydospores formed on hyphae, terminal and intercalary, singly, smooth- and thick-walled, ellipsoidal, 12.3-26.7 × 9.8-17.4 $\mu \mathrm{m}$. Rhizoids present, obvious when growing on glass slides. Zygospores not observed. Maximum growth temperature $37^{\circ} \mathrm{C}$ (without sporulation). Colonies on $P D A$ with uncoloured reverse; sporangia larger (up to $160 \mu \mathrm{m}$ diam). Colonies on SMA with nearly absent aerial mycelium; reverse yellow-orange; sporangia larger (up to $175 \mu \mathrm{m}$ diam); columellae slightly larger (up to $60 \times 62 \mu \mathrm{m}$ ), globose or slightly applanate. Yeast state present in liquid YPG medium.

Habitat — Isolated from Zea mays.

Distribution - South Africa.

Notes - This species is similar to $M$. circinelloides but differs from the latter by forming lighter, slightly orange sporangia and slenderer sporangiospores with a $L / D$ ratio of 1.9 (1.7-2.3) compared to $M$. circinelloides with an $L / D$ ratio of $1.55(1.44-1.73)$

Mucor pseudolusitanicus L. Wagner \& G. Walther, sp. nov. MycoBank MB828293; Fig. 9

Etymology. Refers to its morphological similarity to Mucor lusitanicus.

Typus. GeRmAnY, unknown source and date, unknown collector (holotype CBS H-23930, culture ex-type CBS 540.78)

Colonies on MEA cottony, high, reaching the lid of the Petri dish, fast growing, reaching $5.5 \mathrm{~cm}$ diam after $2 \mathrm{~d}$, young white (10YR 7/1) becoming light grey (10YR 7/2) after $14 \mathrm{~d}$; reverse uncoloured; spores germinate inside sporangia that come in contact with the substrate surface and form small sporangiophores, macroscopically visible as protuberances with up to $1.2 \mathrm{~mm}$ diam (Fig. 9g); reverse spotted by dense areas of the protuberance (Fig. 9b). Sporangiophores of the small and the tall type present; tall sporangiophores repeatedly branched, $10-18.5(-21) \mu \mathrm{m}$ diam, often swollen at the base; small sporangiophores frequently sympodially branched. Sporangia nonapophysate, globose, young hyaline to pale orange, later becoming brown, up to $75 \mu \mathrm{m}$ diam; large sporangia deliquescent; smaller sporangia often still intact after preparation; numerous premature sporangia or sterile sporangia present. Columellae globose, semiglobose or occasionally slightly elongated but not obovoid, large, 35-52 $\mu \mathrm{m}$, hyaline to slightly brown, with distinct collar. Sporangiospores ellipsoidal, varying in size, $4.3-10.3 \times 2.2-6.3 \mu \mathrm{m}$, with a length/width relation of 1.6-2.4, thin-walled, hyaline. Chlamydospores occurring in vegetative hyphae, intercalary, singly, in pairs or in chains, ellipsoidal, 10.4-19.7 × 6.7-15.4 $\mu \mathrm{m}$. Rhizoids present. Zygospores not observed. Maximum growth temperature $35^{\circ} \mathrm{C}$ (without sporulation). Colonies on PDA similar to MEA, sporangia larger (up to $130 \mu \mathrm{m}$ diam); columellae globose to applanate. Colonies on SMA also similar to MEA, sporangia larger (up to $130 \mu \mathrm{m}$ diam); columellae slightly larger (up to $55 \times 57 \mu \mathrm{m}$ ), globose to slightly applanate. Yeast state present in liquid YPG medium.

Habitat — Isolated from sorghum malt.

Distribution - Germany, South Africa.

Additional material examined. SoutH AfrICA, Tijgerberg, sorghum malt, unknown date, C.J. Rabie (CBS 543.80 = MRC 1857)

Notes - Mucor pseudolusitanicus is similar to M. Iusitanicus by the high, grey-brown colonies, the grey-brown sporangia, the predominantly globose columellae and the ellipsoidal sporangiospores. It differs from $M$. lusitanicus by the formation of protuberances and the pale yellow colour of the young sporangia.

The strains CBS 338.71 and CBS 635.65 are closely related to M. pseudolusitanicus and they might also represent undescribed species but we postpone their description because only one strain was available. Strain CBS 338.71 also forms protuberances but it has a higher speed of growth reaching a diam of $9 \mathrm{~cm}$ after $2 \mathrm{~d}$, its maximum growth temperature is $36^{\circ} \mathrm{C}$. 
Strains CBS 635.65 differs from M. pseudolusitanicus by having a slightly orange reverse, by lacking protuberances, by a maximal growth temperature of $33^{\circ} \mathrm{C}$ and by producing also broadly ellipsoidal columellae (Fig. $9 \mathrm{l}-\mathrm{m}$ ). Common to all is that the sporangia of the growing zone are light yellow.

\section{Mucor ramosissimus Samouts., Mater. Mykol. Fitopatol.}

Rossii 6: 210. 1927 - MycoBank MB276099; Fig. 16

Typus. URuguay, H. sapiens (nasal lesion), unknown date, J.E. Mackinnon (neotype CBS H-7376, culture ex-neotype CBS 135.65)

Description \& Illustration - Schipper (1976b).

Habitat - Isolated from grapevine trunks (Kovács et al. 2017, as Mucor sp.), Homo sapiens (Hesseltine \& Ellis 1964). Distribution - Hungary, Uruguay.

Mucor variicolumellatus L. Wagner \& G. Walther, sp. nov. MycoBank MB828292; Fig. 15

Etymology. Refers to the varying shape of its columellae.

Typus. Germany, Hann.-Münden, on Tremella, unknown date, H. Zycha (holotype CBS H-23928, culture ex-type CBS 236.35 = ATCC 10777 = VKM F-1222).

Colonies on MEA cottony, CBS 236.35: high, reaching the lid of the Petri dish; some subcultures of JMRC:SF:012536: medium, 3-4 $\mathrm{mm}$ in height, not reaching the lid, other subcultures higher, reaching the lid in the centre of the culture, fast growing, reaching 4.5-5.0 cm diam after $2 \mathrm{~d}$, young white (5RP 8/1) becoming pale grey (5RP $8 / 2)$, sometimes with tinge of violet especially in the growing zone; reverse uncoloured. Sporangiophores of the small and tall type present; tall sporangiophores repeatedly branched, 10-20(-27) $\mu \mathrm{m}$ diam; small sporangiophores sympodially branched. Sporangia non-apophysate, globose, young hyaline to pale orange, later becoming brown-grey to dark grey, up to $90(-107) \mu \mathrm{m}$ diam, deliquescent. Columella often globose, but also obovoid, ovate or strawberry-shaped, 13.3-47.9(-57) × 13.7-44(-57.8) $\mu \mathrm{m}$, hyaline, with distinct collar. Sporangiospores ellipsoidal, varying in size, 4.2-7.2(-8.8) $\times 2.4-4.5 \mu \mathrm{m}$, with a length/width relation of $1.5-1.8$, thinwalled, hyaline. Chlamydospores intercalary, singly, in pairs or in chains, ellipsoidal or elongated ellipsoidal, 11.3-36.6 × 7-17.2 $\mu \mathrm{m}$. Rhizoids present. Zygospores not observed. Maximum growth temperature $36{ }^{\circ} \mathrm{C}$ (without sporulation). Colonies on $P D A$ grey with a brown tinge; reverse with yellow-orange sectors; sporangia distinctly larger (up to $230 \mu \mathrm{m}$ diam); columellae slightly larger (up to $60 \times 50 \mu \mathrm{m}$ ) than on MEA. Colonies on SMA also grey with a brown tinge; reverse with yellow-orange sectors; sporangia slightly larger (up to $110 \mu \mathrm{m}$ diam), columellae larger (up to $70 \times 63 \mu \mathrm{m}$ ) with a less variable shape (globose to subglobose). Yeast state present in liquid YPG medium.

Habitat - Isolated from Tremella, maize meal, Homo sapiens (Álvarez et al. 2011, as M. fragilis).

Distribution - Germany, Malawi, USA.

Additional material examined. MALAWI, maize meal, 2016, G. Walther (JMRC:SF:012536).

Notes - Mucor variicolumellatus differs from M. Iusitanicus by forming also obovoid, ovate and strawberry-shaped columellae.

\section{Mucor velutinosus E. Álvarez et al., Med. Mycol. 49: 64} 2011 - MycoBank MB539400; Fig. 5

Typus. USA, Connecticut, central venous line site ex joint from $H$. sapiens (holotype CBS H-20399, culture ex-type FMR 10020 = UTHSC 02-1981 = CBS 126272).

Description \& Illustration — Álvarez et al. (2011).
Habitat - Isolated from milk powder, maize meal, dairy product, sediment, insect (Hermet et al. 2012), Homo sapiens (Álvarez et al. 2011), iguana.

Distribution — France, Germany, Malawi, USA, Netherlands.

Additional materials examined. GeRmAny, H. sapiens (sputum), 2012, unknown collector (JMRC:SF:011240). - MALAWI, maize meal, 2016, G. Walther (JMRC:SF:012253). - NETHERLANDS, milk powder, unknown date, unknown collector (CBS 762.74). - UNKNOWN COUNTRY, $H$. sapiens (thigh), unknown date, M.A.A. Schipper \& E.S. van Reenen-Hoekstra (CBS 144.93); iguana lung, 1966, unknown collector (CBS 204.68 = NRRL 3627).

\section{DIAGNOSTIC KEY FOR SPECIES OF MCC BASED ON PHENOTYPE}

The following key of the MCC refers to cultures on MEA (if no other medium is mentioned) incubated at room temperatures and ambient light conditions.

1. Sporangia formed on sympodially branched sporangiophores OR sporangia absent and multispored pedicellate sporangiola developing on irregularly to sympodially branched sporophores; continued growth at $34{ }^{\circ} \mathrm{C}$; in species with high mycelia (reaching the lid in all parts of the Petri dish); columellae of tall sporangiophores without or only with a slightly truncate base (Fig. $3 \mathrm{a}-\mathrm{g}$ ); sporangiospores ellipsoidal with a length to width relation (L/W) of $\geq 1.4$ or subglobose, sporangiophores rarely including chlamydospores. . . . . . . . . . . . . . . . 2 (MCC)

1. Sporangia formed on sympodially and monopodially branched sporangiophores; no continued growth at $34^{\circ} \mathrm{C}$ in most strains (rarely single strains of $M$. racemosus $\mathrm{f}$. racemosus have a Tmax of $34^{\circ} \mathrm{C}$ ); columellae globose, ellipsoidal, hemispherical or obovoid with a distinctly truncate base (Fig. $3 p-w)$; sporangiospores ellipsoidal with a L/W up to 1.35 or subglobose, sporangiophores frequently including chlamydospores................. MRC

2. Red-brown azygospores on branched azygophores present ............. M. bainieri (Fig. 8)

2. Azygospores on branched azygophores absent . . . . 3

3. Sporangiola formed additionally to sporangia or exclusively

$\ldots \ldots \ldots \ldots \ldots \ldots$

3. Sporangiola absent $\ldots \ldots \ldots \ldots \ldots \ldots \ldots \ldots \ldots$

4. No sporangia formed, multispored pedicellate sporangiola develop on irregularly to sympodially branched sporophores Ellisomyces anomalus (Fig. 11, 12b)

4. Terminal sporangia formed, uni- or multispored sporangiola develop often in series laterally on the sporophore. . . . . M. ctenidius (Fig. 12c-d, 18)

5. Colonies flat, velutinous to low cottony (up to $5 \mathrm{~mm}$ in height); aerial mycelium not reaching the lid of the Petri dish; sporangiospores subglobose to irregular or broadly

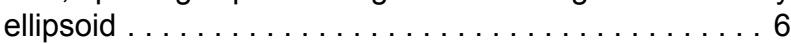

5. Colonies higher, at least in the centre reaching the lid, sporangia brown or black; sporangiospores subglobose, ellipsoid or irregular . . . . . . . . . . . 10

6. Colonies restricted, reaching $<4 \mathrm{~cm}$ diam within $2 \mathrm{~d}$, creamcoloured to grey, sporangia brown-grey or dark brown to black ....................... 7

6. Colonies not restricted, reaching $>4 \mathrm{~cm}$ diam within $2 \mathrm{~d}$, sporangia dark grey to black or violaceus . . . . . . 8

7. Colonies grey when sporangia mature, columella applanate, sterile sporangia frequently formed; sporangiospores slightly angular, subglobose and 4-7(-9) $\mu \mathrm{m}$ diam or short ellipsoidal to irregularly shaped and 4.8-9.5 × 3.7-6.3 $\mu \mathrm{m}$; submersed mycelium with chlamydospores . . . . . . . 
7. Colonies with cream-coloured and grey sectors, columellae light brown, slightly ellipsoid, sphaerical or hemisphaerical, sporangiospores regularly shaped, globose to subglobose, up to 5(-6) $\mu \mathrm{m}$ diam; submersed mycelium without chlamydospores. . . . . . . M. atramentarius sp. nov. (Fig. 13)

8. Columellae of tall sporangiophores globose or rarely elongated, mycelium dark grey to black but especially in the peripheral area violaceus; sporangia large, up to $160 \mu \mathrm{m}$ diam; sporangiospores globose to subglobose, up to $6 \mu \mathrm{m}$ diam. . . . . . . . . M. amethystinus sp. nov. (Fig. 14)

8. Columellae of tall sporangiophores mostly elongated: ellipsoid, obovoid, conical, keyhole-shaped, strawberry-shaped (Fig. 3h-o); mycelium grey to black without violaceus tinge, sporangiospores subglobose . . . . . . . . 9

9. Sporangiospores large, up to $9(-12) \mu \mathrm{m}$ diam; columellae often keyhole-shaped (Fig. 3i, 5h) M. velutinosus (Fig. 5)

9. Sporangiospores small, up to $6 \mu \mathrm{m}$ diam; columellae predominantly obovoid (Fig. $3 f-g$ ), sometimes keyholeshaped (Fig. 3n-o) . . . . . . . . . M. janssenii (Fig. 4)

10. Columellae of tall sporangiophores predominantly globose

Columellae of tall sporangiophores predominantly obovoid

10. Columellae of tall sporangiophores predominantly obovoid

11. Columellae of tall sporangiophores predominantly globose but also obovoid, ovoid and strawberry-shaped columellae formed, Tmax $36{ }^{\circ} \mathrm{C}$

M. variicolumellatus sp. nov. (Fig. 15)

11. Columellae of tall sporangiophores globose, obovoid columellae extremely rare, $\operatorname{Tmax} 35-39^{\circ} \mathrm{C} \ldots \ldots \ldots 12$

12. Sporangiospores either irregularly shaped and large (up to $17 \mu \mathrm{m}$ in length) or ellipsoidal and 4-6.9 $\times 2.6-3.8 \mu \mathrm{m}$, Tmax $35-37^{\circ} \mathrm{C}$, protuberances absent, young sporangia cream-coloured . . . . . . . . . . M. Iusitanicus (Fig. 10)

12. Sporangiospores ellipsoidal to slightly irregularly shaped and $5-7.8 \times 2.2-4.6 \mu \mathrm{m}$, Tmax $\leq 35^{\circ} \mathrm{C}$, protuberances present (Fig. $9 \mathrm{~g}$ ), young sporangia pale orange

M. pseudolusitanicus sp. nov. (Fig. 9)

13. Sporangia dark brown to black (Fig. 7g), Tmax $\leq 36{ }^{\circ} \mathrm{C}$ M. griseocyanus (Fig. 7)

13. Sporangia lighter, brown-grey or light orange, Tmax $\geq 37{ }^{\circ} \mathrm{C}$

14. Sporangia brown-grey, sporangiospores $4.2-9.8 \times 2.7-$ $4.6 \mu \mathrm{m}$ with a mean L/W of $1.55(1.45-1.7)$.

M. circinelloides s.str. (Fig. 6)

14. Sporangia pale orange, sporangiospores $5.2-8.6 \times$ 2.6-3.9 $\mu \mathrm{m}$ with a mean L/W of $1.9(1.7-2.3)$.

M. pseudocircinelloides sp. nov. (Fig. 17)

\section{Clinical relevance and correlation with Tmax}

Studied strains originating from clinical sources are highlighted by black circles in Fig. 2 and belong to $M$. circinelloides, M. griseocyanus, $M$. janssenii, $M$. lusitanicus, $M$. ramosissimus, $M$. variicolumellatus, and $M$. velutinosus. All these species have Tmax of $34{ }^{\circ} \mathrm{C}$ and higher (Table 3 ). The clinically most important species $M$. circinelloides and $M$. velutinosus have the highest Tmax.

\section{DISCUSSION}

Our study provides a revised taxonomy of the MCC based on the first multi-locus phylogeny for this complex. The five-locus phylogeny revealed a higher species diversity in this complex than known before. Five undescribed species were recognised. The renovated molecular species definitions are a prerequisite for correct molecular identification, which in turn is a precondition for insight into ecology, distribution and epidemiology. Our work demonstrates the neglected potential of phenotypic traits for species delimitation, while surprisingly the value of zygospore formation proved to be limited. With the identification key we provide a tool that allows species identification without the use of molecular techniques.

\section{Phenotypic delimitation of the MCC}

The intensive and repeated sympodial branching of sporangiophores of species in MCC and MRC is often used to delimit the M. racemosus group (MCC plus MRC). However, this character has to be used with care especially in older cultures, because sympodial branching also occurs in other Mucor lineages such as in the M. flavus group (Schipper 1978a), the M. hiemalis group (Schipper 1973), in M. fuscus and M. zonatus (Schipper 1973), and occasionally also in the $M$. amphibiorum group (Schipper 1978a).

The presence of short sympodially branched sporangiophores in addition to large and less or non-branched sporangiophores is regarded as a second characteristic of the $M$. racemosus group (Schipper 1976b) but has also been described in the distantly related Mucor species $M$. mucedo, M. piriformis, and M. strictus forming the M. mucedo group (Schipper 1975), and in $M$. aligarensis, M. flavus, $M$. minutus, M. psychrophilus, and $M$. saturninus composing the Mucor flavus group (Schipper 1975, 1978a). Although this feature is less pronounced in these groups it cannot be used alone to define the Mucor racemosus group.

Characteristic of the $M$. racemosus group are the zygospores. When formed on WA they are red-brown to dark brown with a diam of 25 to $80 \mu \mathrm{m}$ (without ornamentation). In contrast to those in other Mucor lineages, zygospores in the M. racemosus group do not become black during maturation (Schipper 1973, 1978b, Schipper et al. 1975).

Thermotolerance is a valuable feature of the MCC because members of many other Mucor lineages, such as the $M$. mucedo group, the $M$. flavus group and the $M$. hiemalis group (with the exception of $M$. irregularis and $M$. endophyticus) do not tolerate temperatures of $34^{\circ} \mathrm{C}$ and above (Schipper 1973, Schipper et al. 1975, Walther et al. 2013). Using traditional morphological features extended with growth velocity and thermotolerance it is possible to identify all MCC species without molecular tools.

\section{CFS - a new marker for mucoralean phylogeny and species identification}

Multi-locus studies of mucoralean phylogeny that include proteincoding genes are often hampered by markers with paralogs that cannot be sequenced directly (Alastruey-Izquierdo et al. 2010, De Souza et al. 2012, Hoffmann et al. 2013, Dolatabadi et al. 2014a). With the introduction of $c f s$ we provide a new single copy marker specific for the Mucorales that has the potential to become one of the standard markers for mucoralean phylogeny. The translation elongation factor $1 \alpha$ (tef) was proposed by Stielow et al. (2015) as universal supplementary barcode to the internal transcribed spacer region bearing high taxonomic and technical applicability to most taxa within Asco- and Basidiomycota. However, this study demonstrate that early diverging lineages of fungi do require a more focused approach with identifying suitable molecular markers for recognising stable taxonomic entities. The issue is particularly relevant to clinical routine, since other widely used identification technologies such as MALDI-TOF instruments (e.g., Biotyper/Vitek2) still perform poorly on Mucoralean taxa, even with updated specialized spectral databases (McMullen et al. 2016). 


\section{Species recognition in the MCC and the value of zygospore formation}

Well-developed zygospores are only formed within the MCC, and not in crosses between members of the MCC and MRC. Zygospores between MCC and MRC are smaller and pale in colour (Schipper 1976b).

In our study we found well-developed zygospores in crosses between species (formerly formae of the M. circinelloides) but our phylogenetic analyses did not detect recombination. The data available do not support a geographical separation of the species that could explain the lack of recombination: $M$. circinelloides, $M$. griseocyanus, $M$. lusitanicus, and $M$. velutinosus are known, for example, from Germany and/or the Netherlands (Walther et al. 2013). Consequently, we suppose a physiological mating barrier: interspecific (formerly interformae) zygospores might not germinate or not undergo meiosis. For M. circinelloides, $M$. griseocyanus, and $M$. lusitanicus we assume that only the large, highly ornamented intraspecific zygospores are able to germinate or to undergo meiosis.

In contrast, the sibling species $M$. janssenii and $M$. velutinosus belong to the same biological species judged by their zygospores: the intraspecific zygospores of $M$. janssenii and of $M$. velutinosus are as small and low ornamented as interspecific zygospores between $M$. janssenii and $M$. circinelloides (Fig. 21d). The numbers of zygospores is similar in crosses within $M$. janssenii and between these species but higher than in crosses with other species (Fig. 21d, Table S3). The species do not seem to have separate areas of distribution: both species were, e.g., isolated in France (Hermet et al. 2012, Walther et al. 2013) but there is no evidence of recombination in our phylogenetic analyses. Morphologically they are clearly differentiated. Based on the number of case reports $M$. velutinosus seems to be the clinically important species (Weitzman et al. 1993 as M. ramosissimus, Álvarez et al. 2009, 2011, Sugui et al. 2011, Wilmer et al. 2013, Joichi et al. 2014, Shelburne et al. 2015, Racsa et al. 2016, Kumar et al. 2018) while there is no case report for $M$. janssenii. Only strain CBS 243.67 is known to be isolated from a case of mucormycosis in South Africa (Walther et al. 2013). Based on these facts two scenarios are possible: 1) recombination occurs between $M$. janssenii and $M$. velutinosus but we did not detect it due to an insufficient number of strains; or 2) no recombination occurs but the mating is aborted to a late time point because the two species separated recently. In our opinion, the benefit of recognising both species in order to detect differences in virulence and distribution justifies the risk that recombination is found if more strains are studied.

Our results show that the simple presence of zygospores cannot be used in the MCC for biological species recognition because also interspecific zygospores are well-developed and can be distinguished from interspecific zygospores only by size and height of ornamentation in comparison to intraspecific zygospores. Schipper (1978a) also found normally shaped zygospores in contrasts of $M$. inaequisporus, $M$. indicus, and $M$. variosporus, species that have a rather different morphology but - as we know today - are closely related, all belonging to the M. amphibiorum group (Walther et al. 2013). Other examples for the development of zygospores between sibling species are Helicostylum pulchrum and $H$. elegans with slightly unequal suspensors compared to those of intraspecific zygospores (Benny 1995), and Blakeslea trispora with its sibling species Gilbertella persicaria (Hesseltine 1960). Crosses between more distantly related species result in azygospore formation, e.g., Gilbertella persicaria with Rhizopus stolonifer (O'Donnell et al. 1977b), and Rhizomucor pusillus with Lichtheimia corymbifera (Schipper 1976a). Thus, in Mucor and other mucoralean genera zygospore formation indicates a close relationship but not necessarily conspecificity. Comparative studies of zygospore quality and quantity are necessary for biological species recognition. In the MCC, low numbers of small zygospores are more likely to represent crosses between different species than within a species.

In M. piriformis, over $70 \%$ of 10 -d-old zygospores were reported to germinate by germ sporangia on acidified medium at 20 to $21^{\circ} \mathrm{C}$ (Guo \& Michailides 1998). In M. hiemalis, however, the percentage of zygospore germination was very low: less than $1 \%$ in 30-90 d, some of them by mycelia but the majority by germ sporangia (Gauger 1965). We only observed germ sporangia of $M$. circinelloides from 10-d-old zygospores and only of a single pair of closely related strains belonging to the same PS. Possibly conditions were not optimal for the germination of zygospores of the MCC and further studies are needed to increase the percentage of zygospore germination.

The diameters of zygospores given by Schipper (1976b) for MCC are larger (80-100 $\mu \mathrm{m})$ compared to our measurements (25-80 $\mu \mathrm{m})$ probably because the ornamentation of the zygospores is included. In addition, whey agar is not a defined medium; it is possible that variations in the medium may influence the size of the zygospores.

If the biological species concept is not entirely applicable for taxonomy within the species complex, the exclusive use of multi-locus phylogeny for species delimitation would probably result in high number of species because of the high molecular diversity in the Mucorales (Walther et al. 2013). However, our study shows that a phylogeny-guided systematic search for group-specific phenotypic characters is successful even in the genus Mucor that is generally regarded as being poor in diagnostic traits. Thus, reasonable species concept in the Mucorales should not only be based on molecular data but also include morphological and physiological features. A biological species recognition that was based on the simple presence of zygospores resulted in broad species concepts used by Walther et al. (2013). The changed concept that includes a comparative study of quality and quantity of zygospores leads to the acceptance of additional species in the MCC. Mucor velutinosus was described from clinical sources by Álvarez et al. (2011). Walther et al. (2013) considered M. velutinosus to be a synonym of $M$. janssenii because the phylogenetic analysis of Álvarez et al. (2011) did not include the most closely related taxon forma janssenii (now M. janssenii), and because of the comparatively small differences in ITS sequences (6 bp). However, multi-locus as well as morphological data clearly show that $M$. velutinosus is a valid species.

Strain CBS 236.35 was isolated by $\mathrm{H}$. Zycha from a Tremella carpophore and mistakenly identified as $M$. fragilis. Walther et al. (2013) assigned the strain to f. Iusitanicus because of the small difference in ITS sequence (7 bp). In his protologue of $M$. fragilis, Bainier (1884) described and illustrated black zygospores and only slightly branched sporangiophores that relate this species to the $M$. hiemalis group. As a consequence, strain CBS 236.35 (PS 11) is described here as a new species, $M$. variicolumellatus. The examples of $M$. variicolumellatus and $M$. velutinosus show that in the derived Mucoraceae subgroups with small ITS differences may represent species. Species diversity might also be higher in other groups of this family for which multi-locus phylogenies are still lacking.

\section{MCC members with deviating morphology}

Based on our five-loci-phylogeny, there is no doubt about the position of Ellisomyces anomalus within the MCC despite its strongly deviating morphology. Interestingly, the multispored sporangiola of this species have well-developed columellae, a feature that was already used by Benny \& Benjamin (1975) to transfer it from Thamnidium to Ellisomyces. Branching of its sporophores is sympodial with swollen branches (Fig. 12b). 
Because of its differing morphology we will not transfer it to Mucor even though it phylogenetically clearly belongs to this genus. So far, this species has only been found in California where it seems to be widely distributed (Benny \& Benjamin 1975). Mucor ctenidius develops uni- and few-spored sporangiola with columellae in clusters along the sporangiophore. In strain CBS 433.87 of $M$. ctenidius the sporangiola-forming hyphae are sympodially branched (Fig. 12c). Similar to the development of small sporangiophores in the MCC (Fig. 12a) a septum is often formed underneath the sporangiolum before the next sporangiolum is formed (Fig. 12c).

The formation of sporangiola in addition to sporangia is known from several lineages of Mucor (Walther et al. 2013) and was used to erect new genera besides Mucor, e.g., Backusella (Ellis \& Hesseltine 1969), and Isomucor (De Souza et al. 2012). Consequently, Walther et al. (2013) suggested that the formation of sporangiola in addition to sporangia is a plesiomorphic character in the Mucorales. However, the presence of columellae and the sympodial mode of branching suggest that the sporangiolaforming hyphae in the MCC have evolved from short sympodial sporangiophores or from intensively sympodially branching side branches of the sporangiophores. Nevertheless, this feature alone cannot be used at the generic level.

\section{Clinical relevance and its correlation to Tmax}

Strains originating from clinical sources are highlighted in Fig. 2. Including the clinical isolates reported by Álvarez et al. (2011) and Walther et al. (2013), seven out of 14 species of the MCC are known from clinical cases: $M$. circinelloides, $M$. griseocyanus, $M$. janssenii, M. lusitanicus, $M$. ramosissimus, $M$. variicolumellatus, and $M$. velutinosus.

The ex-neotype strain of $M$. ramosissimus was isolated from a nasal lesion in Uruguay (Hesseltine \& Ellis 1964). Two other reported cases (Vignale et al. 1964, Weitzman et al. 1993) lack molecular identification. Mucor ramosissimus is often misidentified because growth-reduced variants of other Mucor species mimic this species (Walther et al. 2013) and therefore unconfirmed cases have to be considered as doubtful. Interestingly, this species was isolated several times from trunks of grapevines in Hungary (Kovács et al. 2017), as Mucorsp. (GenBank accession numbers KU377221, KU377255, KU377256, KU377257, KU377259, KU377260).

Clinical importance is correlated with Tmax: MCC species involved in infections have a Tmax of $34^{\circ} \mathrm{C}$ and higher. The species with the lowest $\operatorname{Tmax}\left(34-35^{\circ} \mathrm{C}\right), M$. griseocyanus has only been isolated from a nail infection. The ex-neotype strain of $M$. ramosissimus CBS 135.65 had an original Tmax of $36^{\circ} \mathrm{C}$ (Hesseltine \& Ellis 1964) and might be degenerated since that time because we determined a Tmax of $35^{\circ} \mathrm{C}$ for this strain. Systemic infections by MCC members are only known from species with Tmax of $37^{\circ} \mathrm{C}$ and higher: Mucor circinelloides (Dizbay et al. 2009), M. lusitanicus and M. velutinosus (Álvarez et al. 2011) are the only species reported from blood cultures. The highest number of Mucor isolations from blood refer to M. velutinosus isolated in the USA (Álvarez et al. 2011, Racsa et al. 2016, Kumar et al. 2018), Canada (Wilmer et al. 2013) and Japan (Joichi et al. 2014). The species has been isolated in Europe and Africa as well (Table 1) so that a worldwide distribution is likely. In the past, Mucor species involved in infections often lack identification down to the species level (e.g., Skiada et al. 2011) and are thus not interpretable. However, the few data available suggest that there are differences among the species, e.g., predominantly $M$. velutinosus is found in blood cultures. With the new species concept of the MCC a proper molecular and morphological identification of these species is possible without special expertise which will gain our understanding of ecology and pathogenicity in this group.

\section{CONCLUSIONS}

In conclusion, species diversity of the MCC revealed by five-loci phylogeny (cfs, ITS, $m c m 7, r p b 1, t s r 1$ ) and phenotypic characters is distinctly higher than in biological species recognition based on the presence of zygospores only. The obsolete formae of $M$. circinelloides each represent one or two discrete species. In addition, five species are newly described: $M$. amethystinus, M. atramentarius, M. pseudocircinelloides, M. pseudolusitanicus, and $M$. variicolumellatus. All species of the MCC can be distinguished by morphological traits in combination with their maximum growth temperature and the speed of growth. Among the different markers tsr 1 has the highest resolution power but all other markers including the ITS are appropriate for molecular species identification. Based on the new species concept seven species of the MCC are known to cause human infections by molecular identification of the aetiological agent: M. circinelloides, $M$. velutinosus, $M$. griseocyanus, $M$. janssenii, $M$. Iusitanicus, $M$. ramosissimus, and $M$. variicolumellatus, the first two species representing the most important opportunistic species. In the MCC, zygospores formation only shows a close relation of the mated strains but not their belonging to the same species. A detailed study on quantity and quality of zygospores is necessary to use them for biological species recognition.

Acknowledgements We kindly thank Jennifer Born for help with the software $\mathrm{R}$. This project was funded by the Deutsche Forschungsgemeinschaft (DFG grant-No. WA 3518/1-1). Work in the National Reference Center for Invasive Fungal Infections NRZMyk is funded by the Robert-Koch Institut from funds provided by the Federal Ministry for Health (grant-no.: 1369-240). Molecular work performed at Westerdijk Institute (CBS-KNAW) was supported by European Consortium of Microbial Resource Centres (EMbaRC) through funding of the European Community's Seventh Framework Programme (FP7, 2007-2013), Research Infrastructures action, under grant agreement no. FP7-228310. Part of sequencing work in CBS was supported by Fonds Economische Structuurversterking (FES), Dutch Ministry of Education, Culture and Science grant BEK/BPR-2009/137964-U).

\section{REFERENCES}

Abe A, Asano K, Sone T. 2010. A molecular phylogeny-based taxonomy of the genus Rhizopus. Bioscience, Biotechnology, and Biochemistry 74: 1325-1331.

Aguileta G, Marthey S, Chiapello H, et al. 2008. Assessing the performance of single-copy genes for recovering robust phylogenies. Systematic Biology 57: 613-627.

Alastruey-Izquierdo A, Hoffmann K, De Hoog GS, et al. 2010. Species recognition and clinical relevance of the zygomycetous genus Lichtheimia (syn. Absidia pro parte, Mycocladus). Journal of Clinical Microbiology 48: 2154-2170.

Álvarez E, Cano J, Stchigel AM, et al. 2011. Two new species of Mucor from clinical samples. Medical Mycology 49: 62-72.

Álvarez E, Stchigel AM, Cano J, et al. 2010. Molecular phylogenetic diversity of the emerging mucoralean fungus Apophysomyces: proposal of three new species. Revista Iberoamericana de Micología 27: 80-89.

Álvarez E, Sutton DA, Cano J, et al. 2009. Spectrum of zygomycete species identified in clinically significant specimens in the United States. Journal of Clinical Microbiology 47: 1650-1656.

Anonymous. 1990. Munsell soil color charts. Baltimore, Maryland.

Bainier M. 1884. Nouvelles observations sur les zygospores des Mucoriées. Annales Des Sciences Naturelles Botanique 6: 200-214.

Benjamin R, Mehrotra BS. 1963. Obligate azygospore formation in two species of Mucor (Mucorales). Aliso 5: 235-245.

Benny GL. 1995. Observations on Thamnidiaceae. VII. Helicostylum and a new genus Kirkia. Mycologia 87: 253-264.

Benny GL. 2008. Methods used by Dr. R.K. Benjamin, and other mycologists, to isolate zygomycetes. Aliso 26: 37-61.

Benny GL, Benjamin RK. 1975. Observations on Thamnidiaceae (Mucorales). New taxa, new combinations, and notes on selected species. Aliso 8: $301-351$.

Chan-Tack K, Nemoy L, Perencevich L. 2005. Central venous catheterassociated fungemia secondary to mucormycosis. Scandinavian Journal of Infectious Diseases 37: 925-927. 
Chandra S, Woodgyer A. 2002. Primary cutaneous zygomycosis due to Mucor circinelloides. Australasian Journal of Dermatology 43: 39-42.

Chayakulkeeree M, Ghannoum MA, Perfect JR. 2006. Zygomycosis: the re-emerging fungal infection. European Journal of Clinical Microbiology \& Infectious Diseases 25: 215-229.

Connolly JH, Stodart BJ, Ash GJ. 2010. Genotypic analysis of Mucor from Platypus in Australia. Journal of Wildlife Diseases 46: 55-69.

Corrochano LM, Kuo A, Marcet-Houben M, et al. 2016. Expansion of signal transduction pathways in fungi by extensive genome duplication. Current Biology 26: 1577-1584

Crespo A, Lumbsch HT, Mattsson JE, et al. 2007. Testing morphology-based hypotheses of phylogenetic relationships in Parmeliaceae (Ascomycota) using three ribosomal markers and the nuclear RPB1 gene. Molecular Phylogenetics and Evolution 44: 812-824.

De Hoog GS, Gerrits van den Ende AHG. 1998. Molecular diagnostics of clinical strains of filamentous Basidiomycetes. Mycoses 41: 183-189.

De Hoog GS, Guarro J, Gené J, et al. 2016. Atlas of clinical fungi USB Version 4.1. Webmaster Atlas.

De Souza CAF, Lima DX, Gurgel LMS, et al. 2017. Coprophilous Mucorales (ex Zygomycota) from three areas in the semi-arid of Pernambuco, Brazil. Brazilian Journal of Microbiology 48: 79-86.

De Souza JI, Pires-Zottarelli CL, Dos Santos JF, et al. 2012. Isomucor (Mucoromycotina): a new genus from a Cerrado reserve in state of Sao Paulo, Brazil. Mycologia 104: 232-241.

Dettman J, Jacobson D, Taylor J. 2003. A multilocus genealogical approach to phylogenetic species recognition in the model eukaryote Neurospora. Evolution 57: 2703-2720.

Dizbay M, Adisen E, Kustinur S, et al. 2009. Fungemia and cutaneous zygomycosis due to Mucor circinelloides in an intensive care unit patient: case report and review of literature. Japanese Journal of Infectious Diseases 62: $146-148$

Dolatabadi S, De Hoog GS, Meis JF, et al. 2014a. Species boundaries and nomenclature of Rhizopus arrhizus (syn. R. oryzae). Mycoses 57 Suppl. 3: $108-127$.

Dolatabadi S, Walther G, Gerrits van den Ende AHG, et al. 2014b. Diversity and delimitation of Rhizopus microsporus. Fungal Diversity 64: 145-163. Domsch KKH, Gams W, Anderson TH. 1980. Compendium of soil fungi. Academic Press.

Durrell LW, Fleming M. 1966. A new species of Thamnidium from Death Valley, California. Mycologia 58: 797-801.

Ellis JJ, Hesseltine CW. 1969. Two new members of the Mucorales. Mycologia 61: 863-872.

Fingeroth JD, Roth RS, Talcott JA, et al. 1994. Zygomycosis due to Mucor circinelloides in a neutropenic patient receiving chemotherapy for acute myelogenous leukemia. Clinical Infectious Diseases 19: 135-137.

Garcia-Hermoso D, Criscuolo A, Lee SC, et al. 2018. Outbreak of invasive wound mucormycosis in a burn unit due to multiple strains of Mucor circinelloides $\mathrm{f}$. circinelloides resolved by whole-genome sequencing. MBio 9:e00573-18.

Gauger WL. 1961. The germination of zygospores of Rhizopus stolonifer. American Journal of Botany 48: 427-429.

Gauger WL. 1965. The germination of zygospores of Mucor hiemalis. Mycologia 57: 634-641.

Greenberg RN, Scott LJ, Vaughn HH, et al. 2004. Zygomycosis (mucormycosis): emerging clinical importance and new treatments. Current Opinion in Infectious Diseases 17: 517-525.

Guo L-Y, Michailides TJ. 1998. Factors affecting the rate and mode of germination of Mucor piriformis zygospores. Mycological Research 102: 815819

Han B, Kuijpers A, Thanh N, et al. 2004. Mucoraceous moulds involved in the commercial fermentation of sufu pehtze. Antonie Van Leeuwenhoek 85: 253-257.

Hermet A, Meheust D, Mounier J, et al. 2012. Molecular systematics in the genus Mucor with special regards to species encountered in cheese. Fungal Biology 116: 692-705.

Hesseltine CW. 1960. Gilbertella gen. nov. (Mucorales). Bulletin of the Torrey Botanical Club 87: 21-30.

Hesseltine CW, Anderson P. 1956. The genus Thamnidium and a study of the formation of its zygospores. American Journal of Botany 43: 696-703. Hesseltine CW, Ellis JJ. 1964. An interesting species of Mucor, M. ramosissimus. Sabouraudia 3: 151-154.

Hibbett DS, Taylor JW. 2013. Fungal systematics: is a new age of enlightenment at hand? Nature Reviews Microbiology 11: 129-133.

Hoffmann K, Pawłowska J, Walther G, et al. 2013. The family structure of the Mucorales: a synoptic revision based on comprehensive multigenegenealogies. Persoonia 30: 57-76.
Hong SB, Kim DH, Lee M, et al. 2012. Zygomycota associated with traditional meju, a fermented soybean starting material for soy sauce and soybean paste. Journal of Microbiology 50: 386-393.

Houbraken J, Samson RA. 2011. Phylogeny of Penicillium and the segregation of Trichocomaceae into three families. Studies in Mycology 70: 1-51. Huerta-Cepas J, Capella-Gutierrez S, Pryszcz LP, et al. 2014. PhylomeDB v4: zooming into the plurality of evolutionary histories of a genome. Nucleic Acids Research 42: D897-902.

Iwen PC, Sigler L, Noel RK, et al. 2007. Mucor circinelloides was identified by molecular methods as a cause of primary cutaneous zygomycosis. Journal of Clinical Microbiology 45: 636-640.

James FR, Gauger W. 1982. Studies on the genetics of Mucor hiemalis. Mycologia 74: 744-751.

Joichi Y, Chikimatsu I, Yarita K, et al. 2014. Detection of Mucor velutinosus in a blood culture after autologous peripheral blood stem cell transplantation: a pediatric case report. Medical Mycology Journal 55: E43-E48.

Katoh K, Rozewicki J, Yamada KD. 2017. MAFFT online service: multiple sequence alignment, interactive sequence choice and visualization. Briefings in Bioinformatics bbx108: 1-7.

Kauff F, Lutzoni F. 2002. Phylogeny of the Gyalectales and Ostropales (Ascomycota, Fungi): among and within order relationships based on nuclear ribosomal RNA small and large subunits. Molecular Phylogenetics and Evolution 25: 138-156.

Kovács C, Balling P, Bihari Z, et al. 2017. Incidence of grapevine trunk diseases is influenced by soil, topology and vineyard age, but not by Diplodia seriata infection rate in the Tokaj wine region, Hungary. Phytoparasitica 45: 21-32.

Kumar M, Jantausch B, Torres C, et al. 2018. Central line-associated Mucor velutinosus bloodstream infection in an immunocompetent pediatric patient. Journal of the Pediatric Infectious Diseases Society 7: e55-e57.

Lanfear R, Frandsen PB, Wright AM, et al. 2016. PartitionFinder 2: new methods for selecting partitioned models of evolution for molecular and morphological phylogenetic analyses. Molecular Biology and Evolution 34: $772-773$

Lee SC, Billmyre RB, Li A, et al. 2014. Analysis of a food-borne fungal pathogen outbreak: virulence and genome of a Mucor circinelloides isolate from yogurt. MBio 5: e01390-01314.

Li CH, Cervantes M, Springer DJ, et al. 2011. Sporangiospore size dimorphism is linked to virulence of Mucor circinelloides. PLoS Pathogens 7: e1002086.

Link. 1824. Caroli a Linné Species Plantarum exhibentes Plantas Rite Cognitas ad Genera Relatas 6: 86.

Liu XY, Huang H, Zheng RY. 2008. Delimitation of Rhizopus varieties based on IGS rDNA sequences. Sydowia 60: 93-112.

Ma LJ, Ibrahim AS, Skory C, et al. 2009. Genomic analysis of the basal lineage fungus Rhizopus oryzae reveals a whole-genome duplication. PLoS Genetics 5: e1000549.

McMullen AR, Wallace MA, Pincus DH, et al. 2016. Evaluation of the Vitek MS matrix-assisted laser desorption ionization-time of flight mass spectrometry system for identification of clinically relevant filamentous fungi. Journal of Clinical Microbiology 54: 2068-2073.

Miller M, Pfeiffer W, Schwartz T. 2010. Creating the CIPRES Science Gateway for inference of large phylogenetic trees. New Orleans, Louisiana, USA.

Möller EM, Bahnweg G, Sandermann H, et al. 1992. A simple and efficient protocol for isolation of high molecular weight DNA from filamentous fungi, fruit bodies, and infected plant tissues. Nucleic Acids Research 20: 61156116.

Morin-Sardin S, Jany JL, Artigaud S, et al. 2016. Proteomic analysis of the adaptative response of Mucor spp. to cheese environment. Journal of Proteomics 154: 30-39.

Morin-Sardin S, Nodet P, Coton E, et al. 2017. Mucor: a janus-faced fungal genus with human health impact and industrial applications. Fungal Biology Reviews 31: 12-32.

Mousavi B, Costa JM, Arné P, et al. 2018. Occurrence and species distribution of pathogenic Mucorales in unselected soil samples from France. Medical Mycology 56: 315-321.

Nordberg H, Cantor M, Dusheyko S, et al. 2014. The genome portal of the Department of Energy Joint Genome Institute: 2014 updates. Nucleic Acids Research 42: D26-31.

O'Donnell KL, Ellis JJ, Hesseltine CW, et al. 1977a. Morphogenesis of azygospores induced in Gilbertella persicaria $(+)$ by imperfect hybridization with Rhizopus stolonifer (-). Canadian Journal of Botany 55: 2721-2727.

O'Donnell KL, Ellis JJ, Hesseltine CW, et al. 1977b. Zygosporogenesis in Gilbertella persicaria. Canadian Journal of Botany 55: 662-675.

Petrikkos G, Skiada A, Lortholary O, et al. 2012. Epidemiology and clinical manifestations of mucormycosis. Clinical Infectious Diseases 54 Suppl. 1: $\mathrm{S} 23-34$. 
Pitt JI, Hocking AD. 2009. Zygomycetes. In: Pitt JI, Hocking AD (eds), Fungi and food spoilage, Third ed.: 145-167. Springer, New York.

Racsa LD, Willis B, Lockhart SR, et al. 2016. Bloodstream infection caused by Mucor velutinosus. Infectious Diseases in Clinical Practice 24: e3-4.

Schell WA, O'Donnell K, Alspaugh JA. 2011. Heterothallic mating in Mucor irregularis and first isolate of the species outside of Asia. Medical Mycology 49: $714-723$.

Schipper MAA. 1973. A study on variability in Mucor hiemalis and related species. Studies in Mycology 4: 1-40.

Schipper MAA. 1975. On Mucor mucedo, Mucor flavus and related species. Studies in Mycology 12: 1-40.

Schipper MAA. 1976a. Induced azygospore formation in Mucor (Rhizomucor) pusillus by Absidia corymbifera. Antonie Van Leeuwenhoek 42: 141-144.

Schipper MAA. 1976b. Mucor circinelloides, Mucor racemosus and related species. Studies in Mycology 12: 1-38.

Schipper MAA. 1978a. On certain species of Mucor with a key to all accepted species. Studies in Mycology 17: 1-48.

Schipper MAA. 1978b. On the genera Rhizomucor and Parasitella. Studies in Mycology 17: 53-66.

Schipper MAA, Samson RA, Stalpers JA. 1975. Zygospore ornamentation in the genera Mucor and Zygorhynchus. Persoonia 8: 321-328.

Schmitt I, Crespo A, Divakar PK, et al. 2009. New primers for promising singlecopy genes in fungal phylogenetics and systematics. Persoonia 23: 35-40. Schwartze VU, Winter S, Shelest E, et al. 2014. Gene expansion shapes genome architecture in the human pathogen Lichtheimia corymbifera: an evolutionary genomics analysis in the ancient terrestrial Mucorales (Mucoromycotina). PLOS Genetics 10: e1004496.

Shelburne SA, Ajami NJ, Chibucos MC, et al. 2015. Implementation of a pan-genomic approach to investigate holobiont-infecting microbe interaction: a case report of a leukemic patient with invasive mucormycosis. PLoS One 10: e0139851.

Skiada A, Pagano L, Groll A, et al. 2011. Zygomycosis in Europe: analysis of 230 cases accrued by the registry of the European Confederation of Medical Mycology (ECMM) working group on zygomycosis between 2005 and 2007. Clinical Microbiology and Infection 17: 1859-1867.

Snyder AB, Churey JJ, Worobo RW. 2016. Characterization and control of Mucor circinelloides spoilage in yogurt. International Journal of Food Microbiology 228: 14-21.

Stielow JB, Levesque CA, Seifert KA, et al. 2015. One fungus, which genes? Development and assessment of universal primers for potential secondary fungal DNA barcodes. Persoonia 35: 242-263.

Stöver BC, Müller KF. 2010. TreeGraph 2: combining and visualizing evidence from different phylogenetic analyses. BMC Bioinformatics 11: 7.

Sugui JA, Christensen JA, Bennett JE, et al. 2011. Hematogenously disseminated skin disease caused by Mucor velutinosus in a patient with acute myeloid leukemia. Journal of Clinical Microbiology 49: 2728-2732.

Tanabe Y, Saikawa M, Watanabe MM, et al. 2004. Molecular phylogeny of zygomycota based on EF-1 $\alpha$ and RPB1 sequences: limitations and utility of alternative markers to rDNA. Molecular Phylogenetics and Evolution 30: $438-449$

Taylor JW, Jacobson DJ, Kroken S, et al. 2000. Phylogenetic species recognition and species concepts in fungi. Fungal Genetics and Biology 31: 21-32.
Tedder M, Spratt JA, Anstadt MP, et al. 1994. Pulmonary mucormycosis: results of medical and surgical therapy. The Annals of Thoracic Surgery 57: 1044-1050.

Tretter ED, Johnson EM, Wang Y, et al. 2013. Examining new phylogenetic markers to uncover the evolutionary history of early-diverging fungi: comparing MCM7, TSR1 and rRNA genes for single- and multi-gene analyses of the Kickxellomycotina. Persoonia 30: 106-125.

Van Tieghem P. 1875. Nouvelles recherches sur les Mucorinées. Annales Des Sciences Naturelles Botanique sér. 6, 1: 5-175.

Vignale R, Mackinnon J, Casella de Vilaboa E, et al. 1964. Chronic, destructive, mucocutaneous phycomycosis in man. Sabouraudia 3: 143-147.

Vilgalys R, Hester M. 1990. Rapid genetic identification and mapping of enzymatically amplified ribosomal DNA from several Cryptococcus species. Journal of Bacteriology 172: 4238-4246.

Vitrat-Hincky V, Lebeau B, Bozonnet E, et al. 2009. Severe filamentous fungal infections after widespread tissue damage due to traumatic injury: six cases and review of the literature. Scandinavian Journal of Infectious Diseases 41: 491-500.

Walther G, Pawłowska J, Alastruey-Izquierdo A, et al. 2013. DNA barcoding in Mucorales: an inventory of biodiversity. Persoonia 30: 11-47.

Weitzman I, Della-Latta P, Housey G, et al. 1993. Mucor ramosissimus Samutsevitsch isolated from a thigh lesion. Journal of Clinical Microbiology 31: 2523-2525.

White TJ, Bruns T, Lee S, et al. 1990. Amplification and direct sequencing of fungal ribosomal RNA genes for phylogenetics. In: Innis M, Gelfand D, Sninsky J, et al. (eds), PCR protocols: a guide to methods and applications: 315-322. Academic Press, Inc, New York, USA.

Wilmer A, Wright A, Leung V, et al. 2013. Mucor velutinosus central-line associated bloodstream infection. Clinical Microbiology Newsletter 35: 176-177.

\section{Supplementary material}

Table S1 Overview of strains included in mating experiments. The mating type, the total numbers of crosses per strain and in brackets the numbers of positive crosses are given. Crosses are divided based on the species concept of Schipper (1976b) into intra- and interformae crosses and according to the phylogenetic species recognition into intra and inter PS. * mating type defined in this study.

Table S2 Crosses and zygospore formation in the MCC. All confrontations performed are marked with ' $x$ '. Crosses that resulted in zygospore formation are highlighted grey. ${ }^{*}$ - high numbers of progametangia observed. A - formation of azygospores. Matings which are framed black are not included in Fig. 2 because the few formed zygospores were broken during slide preparation (CBS $172.27 \times$ CBS 239.35, CBS $192.68 \times$ CBS 185.68, CMN-CM 3112 $\times$ CBS 116.08) or because zygospores were only formed on MEA (CBS $852.71 \times$ CBS 277.49).

Table S3 Mean diameter, mean height of ornamentation and abundance formed in the crosses. Parameters highlighted in yellow refer to the reference strain pairs. 